\title{
Laboratory investigation of dense non-aqueous phase liquid migration and remediation in mixed wettability soils monitored by pressure transducers
}

\author{
By \\ Michelle Denise Roberge-Butcher

\begin{abstract}
A thesis submitted to
The Faculty of Graduate and Postdoctoral Affairs

In partial fulfillment of the requirements for the degree of

Master of Applied Science

in Environmental Engineering
\end{abstract}

Department of Civil and Environmental Engineering Carleton University

Ottawa-Carleton Institute for Civil and Environmental Engineering

September, 2016

Copyright $(\mathrm{C}$

2016 - Michelle Roberge-Butcher 


\begin{abstract}
A series of dense non-aqueous phase liquid (DNAPL) spills was conducted in a twodimensional sand box filled with a variety of media. At the conclusion of the spill, the soil underwent remediation via a waterflood followed by a surfactant flush. Pressure data recorded during the experiments confirmed that DNAPL will be drawn into NAPL-wet lenses and result in negative capillary pressures. The waterflood had little impact on the NAPL distribution. The surfactant flush did reduce the DNAPL-water interfacial tension allowing some of the DNAPL to drain from the NAPL-wet lenses; however a NAPL-saturated layer remained at the bottom of the lenses. It was hypothesized that thickness of the NAPL-saturated layer that remained reflects the capillary pressure difference between the reduced NAPL-entry pressure of the hydrophilic soil below the lens and the reduced water-entry pressure that would allow the DNAPL to drain and water to enter the hydrophobic sand.
\end{abstract}




\section{ACKNOWLEDGEMENTS}

I would like to express my sincere gratitude to my friends, colleagues and family who were by my side for this journey. I would particularly like to thank my supervisor Dr. Paul van Geel without whom this work never would have been completed. His guidance and patience have been greatly appreciated. I would also like to thank my colleagues and the staff at Carleton University; particularly Marie Tudoret Chow, Stanley Conley, Pierre Trudel, and Jason Arnot. In addition, I would like to acknowledge the help of former colleagues Luz A Puentes Jácome and Farah Hasson.

Funding for this project was provided in part by the National Sciences and Engineering Research Canada (NSERC) of Canada's Strategic Projects Grant, Discovery Grant and Postgraduate Scholarship programs. Additional funding was provided by the Ontario Graduate Scholarship Program and Carleton University.

Finally I would like to thank my parents and my family who have provided unwavering support. Thank you Kevin and Malcolm for your patience and giving me the time to finish this work. Also a great big thank you to my parents, Jean Paul and Heather Roberge, without whom this journey would not have been possible.

I dedicate this work to those who were on this journey with me but could not see its completion. My sister, Nicole, whose unwavering support is so greatly missed and my daughter, Ava, who is so missed. 


\section{TABLE OF CONTENTS}

Abstract $\quad$ ii

Acknowledgements

Table of Contents

List of Tables

List of Figures $\quad$ vi

List of Equations $\quad x$

List of Acronyms $\quad x$

List of Symbols $\quad x$

List of Subscripts

1.0 Introduction 1

1.1 Problem definition 1

1.2 Objectives 3

1.3 Scope of research 4

$\begin{array}{lll}2.0 & \text { Literature Review } & 5\end{array}$

2.1 Nonaqueous phase liquids in the subsurface 5

2.2 Migration of nonaqueous phase liquids 7

2.2.1 Migration of LNAPLs - under ideal conditions 7

2.2.2 Migration of DNAPLs - under ideal conditions 8

2.3 Factors effecting the migration of NAPLs 9

2.4 Wettability 15

$\begin{array}{ll}\text { 2.4.1 Capillary pressure and wettability } & 18\end{array}$

$\begin{array}{ll}\text { 2.4.2 Changes in wettability } & 24\end{array}$ 
2.5 Laboratory versus field dense nonaqueous phase liquids

2.6 Remediation of nonaqueous phase liquids 34

$\begin{array}{lll}\text { 2.6.1 Waterflooding } 36 & 36\end{array}$

2.6.2 Surfactant enhanced remediation 38

2.6.3 Remediation of field NAPLs $\quad 42$

$\begin{array}{lll}2.7 & \text { Imaging analysis } & 44\end{array}$

$\begin{array}{lll}2.8 \text { Summary } & 52\end{array}$

3.0 Materials and methods $\quad 53$

$\begin{array}{lll}3.1 & \text { Sandbox } & 53\end{array}$

$\begin{array}{lll}3.2 & \text { Pressure transducers } & 59\end{array}$

$\begin{array}{lll}3.3 & \text { Nonaqueous phase liquids } & 61\end{array}$

$\begin{array}{ll}3.4 & \text { Surfactant selection }\end{array}$

$\begin{array}{ll}3.5 & \text { Porous material }\end{array}$

3.6 Experimental set-up 65

$\begin{array}{lll}4.0 & \text { Results } & 69\end{array}$

$\begin{array}{lll}4.1 & \text { Homogeneous hydrophilic sand } & 70\end{array}$

4.2 Hydrophilic sand with fine grained lenses $\quad 79$

4.3 Hydrophilic sand with hydrophobic lenses $\quad 84$

$\begin{array}{llr}5.0 & \text { Discussion } & 99\end{array}$

$\begin{array}{lll}6.0 & \text { Conclusions } & 103\end{array}$

$\begin{array}{lll}6.1 \text { Conclusions } & 103\end{array}$

$\begin{array}{lll}6.2 & \text { Recommendations } & 105\end{array}$

$\begin{array}{lll}7.0 & \text { References } & 108\end{array}$ 


\section{List of Tables}

Table 1: Properties of DNAPLs to be used in experiments

\section{List of Figures}

Figure 1: Measurement of contact angle ( () . A NAPL drop surrounded by water on a strongly water-wet surface forms a bead and its contact angle is approximately $0^{\circ}$ (left). When placed on a strongly NAPL-wet surface (right), the drop collapses resulting in a contact angle around $180^{\circ}$. An intermediate-wet surface (middle) also forms a bead, but the contact angle is determined from the force balance between the water and NAPL surface (adapted from Abdallah et al. 2007).

Figure 2: Capillary pressure-saturation curve, of a water-wet medium, showing effects of hysteresis; where $P_{c}$ is the capillary pressure, $S_{w}$ is the wetting fluid saturation, $S_{n w}$ is the non-wetting fluid saturation, $S_{w r}$ is the irreducible wetting phase and $\mathrm{S}_{\text {nwr }}$ is the residual non-wetting phase (adapted from Pankow and Cherry 1996).

Figure 3: Capillary pressure-saturation drainage curve of a water-wet ( - ) and NAPL-wet ( $\cdots \cdot$ ) media. The $\tau$ symbol indicates the value needed to modify the van Genuchten equation per Bradford and Leij (1995).

Figure 4: Methods to determine the USBM and Amott-Harvey wettability indices. The yellow region is the positive and the red is the negative areas of the capillary pressure - saturation curves (adapted from Abdallah et al. 2007).

Figure 5: Assembled box, prior to placement of pressure transducers and porous media.

Figure 6: Back plate of sand box.

Figure 7: Schematic of back plate for sandbox; all holes are 1/4" NPT. The back plate is $64 \mathrm{~cm} \mathrm{X} 64 \mathrm{~cm}$; however, when assemble the visible area is $60 \mathrm{~cm} \mathrm{X} 60 \mathrm{~cm}$.

Figure 8: Schematic of top and bottom spacers; these spacers are identical, except for the placement of corrosion-resistant phosphor bronze woven wire in the $31 / 6$ " NPT holes in the bottom spacer to ensure no porous media escape the sand box; (a) is the top view of the spacer and (b) is the side view. All units in $\mathrm{cm}$ unless otherwise indicated. 
Figure 9: Schematic of side spacer used in the sand box construction. Units are in $\mathrm{cm}$ unless otherwise indicated.

Figure 10: Pressure transducers used in experiments; (A) -2.0 to 2.0 psig and (B) 2.0 to 3.4 psig.

Figure 11: Funnel used to fill box with porous media.

Figure 12: Placement of hydrophobic/heterogeneous lens; the stainless-steel back plate has been replaced by a pane of glass.

Figure 13: Schematic of sand-box packing and placement of pressure transducers. All units in $\mathrm{cm}$.

Figure 14: Image of the box prior to the spill in homogeneous hydrophilic sand, the black lines indicate the rows for which the pressure transducer data will be described.

Figure 15: Position of the laboratory grade dyed TCE (red) after a spill (12 hrs). In the right hand photograph is a hand drawn interpretation of the DNAPL distribution. The dark blue areas are where DNAPL saturation is high and lighter blue areas indicates areas at which the DNAPL saturation is close to the residual value.

Figure 16: The water, DNAPL and capillary pressure for the spill and the corresponding effective water saturation within the row. A: Row 2, B: Row 3 and C: Row 5, as per Figure 14.

Figure 17: Position of the laboratory grade dyed TCE after a waterflood. In the right hand photograph is a hand drawn interpretation of the DNAPL distribution. The dark blue areas are where DNAPL saturation is high and lighter blue areas indicates areas at which the DNAPL saturation is close to the residual value.

Figure 18: The water, DNAPL and capillary pressure for the waterflood and the corresponding effective water saturation within the row. A: Row 2; B: Row 3 and C: Row 5.

Figure 19: Position of the laboratory grade dyed TCE after a surfactant flush. In the right hand photograph is a hand drawn interpretation of the DNAPL distribution. The dark blue areas are where DNAPL saturation is high and lighter blue areas indicates areas at which the DNAPL saturation is close to the residual value. 
Figure 20: The water, DNAPL and capillary pressure for the surfactant flush and the corresponding effective water saturation within the row. A: Row 2; B: Row 3 and C: Row 5.

Figure 21: White foam which formed within the pooled DNAPL at the bottom of the box.

Figure 22: Image of the box prior to the spill in homogeneous hydrophilic sand with fine grained lenses, the lighter coloured areas are the fine grained lenses.

Figure 23: Position of the field DNAPL after a spill in hydrophilic media with fine grained lenses (12 hrs). In the right hand photograph is a hand drawn interpretation of the DNAPL distribution. The dark blue areas are where DNAPL saturation is high and lighter blue areas indicates areas at which the DNAPL saturation is close to the residual value.

Figure 24: Position of the field DNAPL after a waterflood in hydrophilic media with fine grained lenses. In the right hand photograph is a hand drawn interpretation of the DNAPL distribution. The dark blue areas are where DNAPL saturation is high and lighter blue areas indicates areas at which the DNAPL saturation is close to the residual value.

Figure 25: Position of the field DNAPL after a surfactant flush in hydrophilic media with fine grained lenses. In the right hand photograph is a hand drawn interpretation of the DNAPL distribution. The dark blue areas are where DNAPL saturation is high and lighter blue areas indicates areas at which the DNAPL saturation is close to the residual value.

Figure 26: The water, DNAPL and capillary pressure for Row 7 (located $9.5 \mathrm{~cm}$ from the bottom of the box) during the entire experiment. The pressure remained fairly constant throughout which was common for all rows during all trials.

Figure 27: Image of the sand box prior to the spill of field DNAPL in hydrophilic sand containing 3 hydrophobic lenses. Note the lighter material is the hydrophobic sand and the black circle below lens I is the reflection of the camera lens.

Figure 28: Time lapse photography of a spill with hydrophobic lenses. A: Box prior to the spill. B: DNAPL overcomes the entry pressure and begins to invade the underlying hydrophilic soil, photo taken 50 minutes after the spill began. $\mathrm{C}$ : DNAPL begins to invade the top hydrophobic lens, photo taken 80 minutes after the spill began. D: Top hydrophobic lens is saturated and begins to drain into the underlying hydrophilic media; photo taken approximately 4 hours after the spill began. E: DNAPL begins to invade the second hydrophobic lens; photo taken 4 hours and 20 minutes after the spill began. F: Photo taken at the end of the spill period, 13.5 hours after the spill began. 
Figure 29: The water, DNAPL and capillary pressure for the spill and the corresponding effective water saturation within the lenses. A: Lens I; B: Lens II and C: Lens III. Note for lenses I and II the pressure axes in the plots have been truncated to allow better visualization of the data; as the strongly negative pressures of the DNAPL prior to the arrival of the DNAPL do not reflect actual DNAPL pressures.

Figure 30: Position of the field DNAPL after a spill (12 hrs). In the right hand photograph is a hand drawn interpretation of the DNAPL distribution. The dark blue areas are where DNAPL saturation is high and the lighter blue indicates areas at which the DNAPL saturation is close to the residual value.

Figure 31: Schematic of the estimated pressure distribution in lens I after the spill.

Figure 32: The water, DNAPL and capillary pressure for the waterflood and the corresponding effective water saturation within the lenses. A: Lens I; B: Lens II and C: Lens III.

Figure 33: Position of the field DNAPL after the waterflood. In the right hand photograph is a hand drawn interpretation of the DNAPL distribution. The dark blue areas are where DNAPL saturation is high and the lighter blue indicates areas at which the DNAPL saturation is close to the residual value.

Figure 34: Time lapse photography of a surfactant flush with hydrophobic lenses. A: Box at the end of the waterflood and prior to the surfactant flush. B: The saturated top lens begins to drain again into the underlying hydrophilic soil; this photo was taken approximately 3 hours after the previous photo. C: The DNAPL pool at the interface between the water and porous media begins to drain, photo taken 3.5 hours after the previous photo. D: The second lens begins to drain to drain into the underlying hydrophobic media, photo taken 1 hour after the previous photo. E: Photo taken at the end of the surfactant flood, 7 hours after the previous photo.

Figure 35: The water, DNAPL and capillary pressure for the surfactant flush and the corresponding effective water saturation within the lenses. A: Lens I; B: Lens II and C: Lens III.

Figure 36: Position of the field DNAPL after a surfactant flush. In the right hand photograph is a hand drawn interpretation of the DNAPL distribution. The dark blue areas are where DNAPL saturation is high, the medium blue designates areas within the hydrophobic lenses from which the DNAPL has drained and the lighter blue indicates areas at which the DNAPL saturation is close to the residual value. 


\section{List of Equations}

$\begin{array}{llr}3.1 & \text { Laplace equation for capillary pressure } & 18\end{array}$

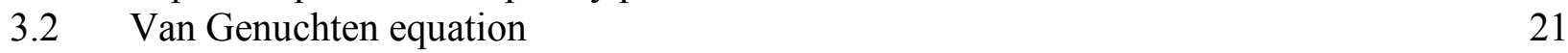

3.3 Van Genuchten equation modified to account for presence of NAPL-wet 21 porous media

$\begin{array}{lll}3.4 & \text { U.S. Bureau of Mines index to determine wettability } & 22\end{array}$

3.5 Amott-Harvey index to determine wettability 23

3.6 Mixing theory to calculate interfacial tension 30

$\begin{array}{lll}3.7 & \text { Trapping number } & 35\end{array}$

$\begin{array}{lll}3.8 & \text { Capillary number } & 35\end{array}$

$\begin{array}{ll}3.9 & \text { Bond number } \\ 3.10 & \text { Dip angle }\end{array}$

$\begin{array}{ll}3.10 & \text { Dip angle }\end{array}$

CCD - Charged coupled device

\section{Acronyms}

$\mathrm{CHC}$ - Chlorinated hydrocarbon

$\mathrm{CMC}$ - Critical micelle concentration

CMOS - Complimentary metal-oxide semiconductor

CTAB - Cetyltrimethylammonium bromide

DDA - Dodecylamine

DNAPL - Dense nonaqueous phase liquid

IFT - Interfacial tension

LNAPL - Light nonaqueous phase liquid

NAPL - Nonaqueous phase liquid

OTS - Octadecyltrichlorosilane

PBA - Polybuteneamine

PCE - Tetrachloroethylene

SEAR - Surfactant enhanced aquifer remediation

TCE - Trichloroethylene

TCA - Trichloroethane

USBM - U.S. Bureau of Mines 
$\mathrm{P}$ - Pressure

\section{Symbols}

$\mathrm{S}$ - Saturation

$\sigma-$ Interfacial tension

$\mathrm{r}-$ Radius

$\Theta-$ Contact angle

$\alpha-$ Fitting parameter for van Genuchten equation

$\mathrm{n}$ - Fitting parameter for van Genuchten equation

$\mathrm{S}$ - Saturation

$\bar{S}$ - Effective saturation

$\tau-$ Fitting parameter to ensure positive capillary pressure

A - Area

$\mathrm{X}$ - Mole fraction

$\mu-$ Viscosity

q - Darcy flow

$\mathrm{k}$ - permeability

$\mathbf{k}$ - Intrinsic permeability

$\mathrm{k}_{\mathrm{r}}$ - Effective permeability

$\mathrm{N}$ - Capillary number

$\mathrm{N}_{\mathrm{T}}$ - Trapping number

$\mathrm{N}_{\mathrm{B}}$ - Bond number

$\gamma-$ Dip (angle of mobilized DNAPL)

c - Capillary

\section{Subscripts}

w - Wetting

nw - Non-wetting

ir - Irreducible

s - Saturated

i - Phase

app - Apparent

eff - Effective 


\section{INTRODUCTION}

\subsection{Problem definition}

Common contaminants found at polluted sites are non-aqueous phase liquids (NAPLs). There are many types of NAPLs found in the environment and these contaminants are usually subdivided into two categories: light non-aqueous phase liquid (LNAPL) or dense non-aqueous phase liquid (DNAPL). When spilled on unsaturated soils both LNAPLs and DNAPLs will move through the unsaturated zone; however, their behaviour differs when in contact with the saturated zone. LNAPLs, which are less dense than water, will float when in contact with the water table. In contrast, DNAPLs, which are denser than water, will move down through the saturated zone until they come into contact with an impermeable boundary. As a result, DNAPLs can migrate a significant distance in the subsurface.

As a large number of sites are contaminated with NAPLs and due to their potentially high toxicity, an understanding of the migration of these chemicals in the subsurface is of great importance. It is extremely difficult to monitor a NAPL spill occurring in the field; therefore, the majority of research on the movement of NAPLs has taken place under laboratory conditions. These laboratory scale experiments include pore-scale micromodels, one-dimensional column experiments and multidimensional aquifer models, which can be two- or three- dimensions (Oostrom et al. 2007). In the majority of the multidimensional models, at least one side of the enclosure is composed of a transparent material, which allows for monitoring of a NAPL spill and in many instances 
the spill is modelled as well (Darnault et al. 1998; Kueper et al. 1989; Conrad et al. 2002).

Recent models examining NAPL spills have focused on the effects of soil wettability on their movement in the subsurface. Originally it was assumed that all sands were water-wet (hydrophilic). In hydrophilic porous material, the grains are first coated by water, then the NAPL and finally air as the least wetting phase. However, research has shown that some materials are naturally intermediate- or NAPL-wet or they can become so through long-term exposure to NAPLs (Denekas et al. 1959; Powers and Tamblin 1995; Powers et al. 1996; Abdallah et al. 2007). This change in wettability can have dramatic effects on the movement of the NAPL through the subsurface.

Early research examining the migration of NAPLs in the subsurface was focused on the removal of oil from reservoir rocks (Denekas et al. 1959). This research was quickly converted into use for the removal of LNAPLs from the subsurface; in fact, many of the methodologies used for contaminant clean-up have a basis in reservoir rock engineering. Two common remediation techniques are waterflooding and surfactant flushing. In waterflooding, large volumes of water are pumped through areas of contamination in an attempt to remove the contamination. Surfactant flushing is similar to water flooding, although a surface active agent (i.e. surfactant) is added to the water which is pumped into the subsurface. The surfactant acts by decreasing the interfacial tension (IFT) between a NAPL and the aqueous phase and can be used to increase the solubility of NAPLs in water.

In many cases, when remediation techniques are first attempted in a laboratory setting, the results appeared promising; however, the results of field tests were not as 
encouraging (Oostrom et al. 1999a \& b; Suchomel et al. 2007). The exact reasons for the discrepancy between the laboratory and field results are unknown, but possible reasons include the porous media and NAPL used in the simulated spills. Under laboratory conditions, spills are usually conducted with one neat (pure) laboratory grade NAPL and clean, very well characterized, water-wet sand. Under field conditions, the NAPL is usually a blend of different NAPLs, surfactants and oils. These additions can alter the properties of the original NAPL. Porous material recovered from the site of a previous spill location is rarely in pristine condition. It may have been in contact with the NAPL for a long period of time which can alter the wettability of the soil, the media may also have a coating of organic material which may affect the retention of the NAPL, and/or the soil could naturally be intermediate- or NAPL-wet (Powers and Tamblin 1995; Powers et al. 1996).

\subsection{Objectives}

The objective of this study was to use pressure transducers and photographic evidence to examine the impacts of wettability on the migration and remediation of DNAPLs under a variety of different spill conditions. A variety of soils and DNAPLs, both laboratory grade and field were used to compare traditional lab experiments with strongly water-wet soils and neat, lab-grade DNAPLs with experiments that use porous media with differing wettability conditions and a field DNAPL. DNAPLs were spilled in a two-

dimensional sandbox and monitored through use of photography and pressure transducers. After the spill the porous media were remediated by a waterflood followed by a surfactant flush. The use of pressure transducers within hydrophobic soils is novel 
and this research will improve our understanding of the migration and subsequent remediation of DNAPL spills in soils with NAPL wet lenses. In particular the pressure transducer data will convey how the capillary pressure changes as the DNAPL front migrates through the box and enters NAPL-wet lenses and how the NAPL-wet lenses impact the migration and remediation of DNAPLs in the subsurface.

\subsection{Scope of research}

This research was conducted in order to better understand the behaviour of DNAPLs during their migration and subsequent remediation under a variety of conditions through the use of pressure transducers and photographic evidence. The DNAPLs used in these experiments were a pure laboratory grade trichloroethylene (TCE) and a field DNAPL collected from a site in Ottawa, Ontario. These DNAPLs were spilled in 2-D sand box using a variety of media:

- Homogenous water-wet sand,

- Homogenous water-wet sand with lenses of fine grade water-wet material, - Homogenous water-wet sands with lenses of NAPL-wet sand.

The spills were then subsequently remediated using a waterflood which was immediately followed by a surfactant flush. 


\section{LITERATURE REVIEW}

\subsection{Non-aqueous phase liquids in the subsurface}

Non-aqueous phase liquids (NAPLs) are common contaminants found at brownfield sites and dissolved in groundwater. They enter the subsurface through leaking underground storage containers, improper storage and surface spills. Some of the released NAPL can enter pore spaces where it can be trapped and, as NAPLs have a low solubility, can act as a long term source for further contamination via dissolution to the aqueous phase (Bedient et al. 1997; Powers et al. 1996).

Non-aqueous phase liquids are of concern as they are hazardous even in minute concentrations. For example, Health Canada has specified a maximum allowable drinking water concentration of only $5 \mu \mathrm{g} / \mathrm{L}$ for benzene, a chemical found in crude oil and gasoline, and $30 \mu \mathrm{g} / \mathrm{L}$ for tetrachloroethylene (PCE), a solvent commonly used in dry-cleaning applications (Health Canada 2008). At these minuscule allowable maximum concentrations, even small spills can be problematic and trapped residuals can act as long term sources of contamination.

In addition to being used in a wide variety of applications, one reason for the large number of sites contaminated with NAPLs is the high volatilities of these compounds. When these compounds were first in use, the typical method of disposal was to release these chemicals onto dry ground and allow the spill to volatilize. However, not all the NAPL would evaporate and some would enter the saturated zone where its high volatilization rate was of little use (Pankow and Cherry 1996). Once NAPLs are in the 
subsurface, they have the added issue of being immiscible in water; however, this statement is misleading as NAPLs do dissolve in water just with a very low solubility limit. For example, trichloroethylene (TCE) has a solubility limit of $1,100 \mathrm{mg} / \mathrm{L}$ (Pankow and Cherry 1996). Furthermore, many of these chemicals tend to have very low degradability. All of these factors lead to NAPLs acting as sources of groundwater contamination for many years and can make the remediation of sites with these contaminants difficult. In fact, it has been suggested that for complete remediation, a minimum of $99 \%$ of the original volume of a contaminant spill needs to be removed from the subsurface (Pankow and Cherry 1996; Bedient et al. 1997). However, others have found that removal of the source zone itself is often not enough to ensure that the subsurface contamination levels can be reduce to achieve drinking water standards. Seyedabbasi et al. (2012) found that through dissolution alone a TCE source zone could be removed within 38 years; however, the back diffusion of the TCE from lower permeable material can extend the longevity of these sources of contamination for 83 years. These results were similar to those of Wilking et al. (2013), who found that DNAPL spills will persist in areas with low permeability zones regardless of the level of DNAPL saturation at the interface between the low and highly permeable media. A high level of saturation at this interface causes flow bypass and the penetration into the low permeability media due to diffusion. If the level of DNAPL saturation was low it resulted in the deeper penetration of the DNAPL into the lower permeable media due to the effects of advection and diffusion. 


\subsection{Migration of non-aqueous phase liquids}

NAPLs can be subdivided into two categories: light non-aqueous phase liquids (LNAPs) and dense non-aqueous phase liquids (DNAPLs). LNAPLs will float on water as they have a specific gravity which is less than that of water. As LNAPLs do not normally penetrate deeply into a water table and are often biodegradable, they are thought to represent a lower environmental risk than a DNAPL release (Bedient et al. 1997). DNAPLs are denser than water and can penetrate an aquifer for some depth until the migration is halted by an impermeable barrier. NAPLs are produced and used in a wide variety of activities and processes. Of particular concern are chlorinated solvents and other organics (Bedient et al. 1997). The following two sections describe the movement of LNAPLs and DNAPLs under ideal conditions, which assumes that the spills are large and the porous material is hydrophilic, meaning that water is the most wetting fluid, followed by the NAPL with air as the least wetting. If the spill is small, all NAPL is held in pores in the unsaturated zone and the NAPL does not come into contact with the water table.

\subsubsection{Migration of light non-aqueous phase liquids (under idealized conditions)}

When LNAPLs are released at the surface or in the unsaturated zone, they immediately begin to migrate vertically due to the effects of gravity. As the LNAPL travels downward, some remains as residual in the pore spaces due to capillary forces and is no longer mobile. Once the mobile fraction of the LNAPL is in contact with the capillary fringe, the capillary fringe will act as a capillary barrier due to buoyancy forces. 
At this barrier, the LNAPL begins to accumulate and flow laterally along the surface of the water particularly in the direction of groundwater flow. However, the LNAPL will cause some collapse to the capillary fringe, which may allow the LNAPL to penetrate a slight distance into the saturated zone (Bedient et al. 1997). Once the source is exhausted, and the LNAPL is no longer accumulating at the capillary fringe, the buoyancy forces of the water will force the mobile portion of the LNAPL to the surface and only the trapped residual will be left in the saturated zone (Mayer and Hassanizadeh 2005).

The most significant problem with LNAPL contamination is the possibility of a smear zone (Mayer and Hassanizadeh 2005). A smear zone develops when the water table drops and/or rises. If the water table is lowered some LNAPL will remain in the pore spaces in the unsaturated zone and the mobile LNAPL can penetrate into areas which were formerly uncontaminated. With a rising water table, the mobile phase will follow the water table with some LNAPL trapped in the saturated zone. This results in conditions which make remediation considerably more difficult.

\subsubsection{Migration of dense non-aqueous phase liquids (under idealized conditions)}

When DNAPLs are released to the unsaturated zone, they will also migrate downward; however, unlike LNAPLs they continue the downward migration through the capillary fringe until the mobile source is exhausted or a low permeability layer is encountered. As the DNAPL migrates downward, residual and entrapped DNAPL remains in some pores in the unsaturated and saturated zones respectively. These 
residuals and entrapped DNAPLs result from capillary forces that occur at the interface between the DNAPL and water, and the surface tension that arises between the DNAPL and air. The amount of residual and entrapped DNAPL which is retained in the subsurface depends on a variety of factors but typically between 5 to 20 percent of the pore spaces are filled by DNAPL (Kueper et al. 2003).

Unlike an LNAPL, the mobile phase of a DNAPL spill will continue to migrate downward until it reaches the capillary fringe at which point the DNAPL will enter the saturated zone. However, this will only occur when enough head is built up to overcome the entry pressure of the soil. Therefore, there can be some lateral spreading at this point as the DNAPL pools on the water surface. If the lateral spreading is not too extensive and enough DNAPL pools to generate the necessary head, it will enter the saturated zone. Once the DNAPL has entered the saturated zone, it will continue to migrate downward until it comes into contact with a low permeability layer at which point the DNAPL will pool. This lower permeability material provides a capillary barrier and, as with the water table, the DNAPL will begin to spread laterally across this barrier (Bradford et al. 1998; Dekker and Abriola 2000). All these factors make it difficult to determine the lateral and vertical extent of DNAPL migration.

\subsection{Factors affecting the migration of NAPLs}

The migration of NAPLs as described in the previous two sections are simplified conceptual representations which occur under specific, ideal conditions which are not often found in the field. Actual migration of NAPLs in the subsurface is controlled by a 
combination of physiochemical properties and geological structures. Initial water saturation, fluid properties, soil heterogeneities and wettability are among the factors that can have drastic impacts on NAPL migration (Dekker and Abriola 2000).

Early work in multiphase flow assumed ideal subsurface conditions, including homogeneous, water-wet porous media (Bedient et al. 1997; Oostrom et al. 2007). However, more recent research has shown the importance of the inclusion of heterogeneous formations, and the resulting variability in permeability and capillary pressure-saturation relationships, in NAPL migration (Kueper et al. 1989; Bradford et al. 1998; Miller et al. 1998; Conrad et al. 2002).

The first in depth studies examining the migration of DNAPLs in the subsurface were conducted by Schwille beginning in 1975 (Schwille 1988). Schwille ran experiments to determine if the use of theoretical hypotheses could be used to predict the behaviour of chlorinated hydrocarbons (CHCs) in the subsurface. Early results were as expected, the CHCs penetrated unsaturated porous media more quickly than water. When the capillary fringe was reached, the water did restrict the movement of the $\mathrm{CHC}$ downward, but did not halt it completely and the downward migration continued after the entry pressure was attained. The permeability of the porous media controlled the speed of the penetration of the DNAPL, as the higher the permeability the more quickly the CHC penetrated the media and in the lower permeability material the sinking of the $\mathrm{CHC}$ was retarded and even stopped. After the small-scale experiments, Schwille moved to large-scale trough experiments in which TCE was spilled into a model groundwater aquifer containing two different layers, a less permeable porous layer $\left(8 \times 10^{-4} \mathrm{~m} / \mathrm{s}\right)$ over a more permeable one $\left(3.2 \times 10^{-3} \mathrm{~m} / \mathrm{s}\right)$. In the first experiment, the water table was located in the upper, less 
permeable layer. Contact with the saturated zone resulted in a slight delay in the downward migration of the TCE and a slight lateral spread. However, as the TCE continued its downward migration, the lateral spread decreased as the TCE penetrated the more permeable layer and did not encounter the same resistance in entering the pore spaces. Eventually, the bottom of the trough halted the downward movement of the fluid, but it pooled in this location and spread laterally along the bottom. In the second experiment, the saturated zone was located in the more permeable layer. This resulted in a spill that migrated downward with no lateral spread until it came into contact with the capillary fringe. The spill then experienced a slight lateral spread and continued to migrate downward until the bottom of the trough was reached. In addition, groundwater flow did not significantly affect the distribution of the TCE. The results of these experiments demonstrate that with an adequate infiltration rate and high soil permeability, a $\mathrm{CHC}$ can quickly penetrate an aquifer; however, saturated low permeability material can impede or completely halt the downward migration of DNAPL.

In order to determine the relationship between pore size and entry pressure of the DNAPL, Schwille (1988) organized a series of glass frit experiments. The results indicate that to enter water saturated pores, the entry pressures required increases quickly as the pore size drops below $0.5 \mathrm{~mm}$. Macroscopic experiments were also conducted to determine what happens at the pore level as a $\mathrm{CHC}$ infiltrates pore spaces. If the pore spaces contain water, the $\mathrm{CHC}$ can only enter pores which are not completely saturated. In a dry porous medium, the $\mathrm{CHC}$ invades the pore spaces, but if water is then added, it begins to displace the $\mathrm{CHC}$ and the water is then wetting to the beads. After the spill has ceased, larger pools of $\mathrm{CHC}$ may exist and some will remain entrapped in the pore 
spaces. Groundwater flow can eventually remove the $\mathrm{CHC}$; however, the entrapped phase cannot be displaced as a mobile phase and remains trapped in the pore spaces. Removal only occurs as the $\mathrm{CHC}$ dissolves and diffuses into the surrounding water and the rate of dissolution depends on the solubility limit of the DNAPL and groundwater flow rate. In addition, $\mathrm{CHCs}$ are lipophilic and as such will easily adsorb onto soils with high organic content making their removal even more difficult.

When DNAPL spills were modelled by Dekker and Abriola (2000), they found that if the heterogeneity, treated as the variance of intrinsic permeability in the model, was increased the model produced results indicating that more lateral spread would occur; maximum DNAPL saturation levels would be greater and the depth of penetration of the spill would not be as great. In addition, the variability between trials with increased heterogeneity was greater. They also found that the depth and extent of migration of a DNAPL spill in the subsurface is influenced by spill rate. Increasing the spill rate resulted in an increased maximum DNAPL saturation, a decrease in the depth of penetration and decrease in the lateral spread of DNAPL. However, the effects of spill rate are diminished by changes in intrinsic permeability, suggesting that in a heterogeneous formation, the spill rate will not likely be an important parameter in determining spill behaviour. The authors also examined the effect of capillary pressuresaturation $\left(\mathrm{P}_{\mathrm{c}}-\mathrm{S}\right)$ relationships scaled with permeability and found that changes to the permeability field did change the $\mathrm{P}_{\mathrm{c}}$-S relationship but the type of scaling also influenced the results. The model results further implied that spill migration was not affected by entrapped DNAPL and water saturation. However, the authors did stress that they used 
the van Genuchten equation to determine the $\mathrm{P}_{\mathrm{c}}$-S relationship and the results could change if an alternative function were used.

Kueper et al. (1989) ran a sandbox experiment containing four sands of different permeabilities to examine the effects of heterogeneity, and the associated capillary effects, on the migration of TCE. They investigated the infiltration of the DNAPL into groundwater where there had previously been no DNAPL, the lateral flow of the DNAPL along low permeability layers and the vertical build-up of DNAPL on these layers, and the subsequent penetration when sufficient displacement pressure was generated. Immediately after injection, the TCE began to migrate downward and exhibit some minor lateral movement in the most permeable sand which made up the bulk of porous media in the box. The authors believed that the lateral movement at this stage of the experiment was likely due to the arrangement of sand grains in the box. When the TCE reached a lens of finer porous material, it began to pool and spread laterally across the lens surface until it reached the edges of the lens at which point the TCE began to cascade over the sides and continue to migrate downward. During the course of the experiment, the only lens which was penetrated by the TCE was composed of the most permeable lens material, as this was the only material for which the vertical build-up of TCE generated enough capillary pressure to overcome the displacement (or entry) pressure. Once the TCE permeated the lens, it began to flow laterally and vertically through the lens. Although the TCE did penetrate this lens, it also continued to pool on the surface of the lens. At no time during the experiment did the TCE penetrate into the remaining two less permeable sands. 
Oostrom et al. (1999a \& b) conducted flow cell experiments in order to monitor spill behaviour and remediation of a TCE spill in a saturated, heterogeneous porous medium. The authors found that the TCE spill initially progressed as thin fingers through the coarse-grained material until it reached a fine-grained lens at which point it began to accumulate and spread laterally across the surface of the lens. At no point did the TCE penetrate this lens although the TCE did cascade over the edges of the lens. When the TCE spill encountered the medium-grained lenses, it also began to accumulate; however, a close visual inspection and later gamma radiation readings indicated that the TCE did penetrate these lenses. The majority of the TCE spilled accumulated at the top of the layer of fine-grained material at the bottom of the tank. Sampling ports in the flow cells allowed for sampling of the dissolved TCE concentrations which indicated that the concentrations were well below the solubility limit of TCE. This is a signal that the groundwater flow rate was quite high and/or the possibility of rate limited mass transfer. In addition, the concentration measurements suggest that abnormally large vertical dispersivity (greater than $0.005 \mathrm{~m}$ ) would be required to generate the concentrations recorded. These results suggest that the dissolved TCE concentrations measured are the result of not only dissolution from the pooled TCE but also the entrapped TCE within the flow cell. 


\subsection{Wettability}

Wettability is the property that describes the preference for a fluid to spread across a solid surface when in contact with other fluids (Abdallah et al. 2007). When a fluid is the wetting fluid, it will spread across the solid surface and will displace a less wetting fluid. In contrast, if a non-wetting fluid is applied to a solid surface, in the presence of a more wetting fluid, it will form a bead in an attempt to minimize its contact with the surface. The interface between the solid surface and the two fluids forms an angle known as the contact angle; this angle is used as a measure of wettability. As seen in Figure 1, the contact angle is commonly measured through the water. A small contact angle (usually less that $75^{\circ}$ ) indicates that a surface is strongly water wetting, a large angle (between $180^{\circ}$ and $105^{\circ}$ to $120^{\circ}$ ) denotes a strongly NAPL wetting surface and an angle near $90^{\circ}$ indicates the surface is neutral or intermediate wetting (Anderson 1987a; Abdallah et al. 2007). The actual cut-off angle for each wetting classification varies between authors (Powers et al. 1996).

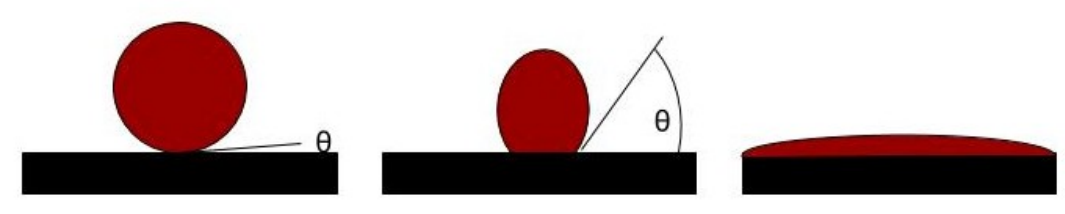

Figure 1: Measurement of contact angle ( $\theta$ ). A NAPL drop surrounded by water on a strongly water-wet surface forms a bead and its contact angle is approximately $0^{\circ}$ (left). When placed on a strongly NAPL-wet surface (right), the drop collapses resulting in a contact angle around $180^{\circ}$. An intermediate-wet surface (middle) also forms a bead, but the contact angle is determined from the force balance between the water and NAPL surface (adapted from Abdallah et al. 2007). 
Porous media which have water as the preferential wetting fluid are known as waterwet or hydrophilic and porous media with NAPL as the favoured wetting fluid are known as NAPL-wet, oil-wet or hydrophobic. Many surfaces display an intermediate wetting surface; they are neither strongly water-wet nor NAPL-wet. When the migration of NAPLs was first investigated, it was assumed that all subsurface porous media were water-wet. This would mean that water would be the fluid closest to the soil grains, followed by the NAPL, with air as the least wetting fluid. However, as early as 1959, petroleum engineers recognized that exposure to crude oil can alter the wettability of porous media (Denkas et al. 1959). It is now recognized that in the field porous media can range from strongly water-wet to strongly NAPL-wet (Powers and Tamblin 1995; Powers et al. 1996; Abdallah et al. 2007).

Differences in wettability have significant effects on the distribution of NAPLs in the subsurface (O'Carroll et al. 2004; Abdallah et al. 2007). In a water-wet medium, the smallest pores are filled with water, which also coats the soil grains, and the larger pores tend to be occupied by NAPLs. This inclination is reversed in a NAPL-wet medium, with the largest pores containing water and the NAPL is found in the smaller pores and coating the soil grains.

One important factor to note when describing wettability is that it refers to a medium's wetting preference, not the fluid which may be saturating the porous material (Abdallah et al. 2007). A medium which is in fact water-wet may be saturated by NAPL and vice versa; however, when in contact with the fluid of preference, the favoured fluid will be imbibed and the less preferred fluid expelled. 
Most research studies into the migration of NAPLs in the subsurface have used porous media which are either strongly water-wet or, less often, strongly NAPL-wet. However, in the natural environment, it is most likely that the subsurface is a combination of the two at varying strength, known as a fractionally wet system. AlRaoush (2009) investigated the impact of different proportions of NAPL-wet media (100, 50, 25 and $0 \%$ NAPL-wet fractions) on the pore-scale characteristics of entrapped NAPLs. He found that the percentage of NAPL-wet media has a significant impact on the distribution of entrapped NAPL. With an increasing proportion of water-wet material, the size and length of entrapped NAPL blobs increased as a result of entrapment in the pore spaces. Generally, the smallest entrapped NAPL saturations were found in the fractionally wet systems, with the entrapped NAPL increasing as the system moved towards a completely NAPL- or water-wet system. O'Carroll et al. (2004) conducted a series of flow cell experiments to model the effects of having lenses of different wettability and pore sizes on NAPL migration. During these experiments, lenses of finegrained water-wet material acted as barriers, inhibiting the downward migration of the NAPL. However, PCE did spontaneously imbibe into the NAPL-wet lenses and resulted in the retardation of the downward migration of the NAPL. These results show that the presence of media of differing wettability and permeability can have significant impacts on the migration of NAPLs in the subsurface. 


\subsubsection{Capillary pressure and wettability}

Capillary pressure is the pressure jump which occurs at an interface in order to balance the interfacial tension (IFT) forces and it is affected by the local pore geometry, wettability, saturation and saturation history (Anderson 1987a). Interfacial tension occurs at the interface between two immiscible fluids and is the result of the increase in potential energy of the molecules at the interface (Harrold et al. 2003). In a multiphase system, capillary pressure can be expressed as the pressure at which a non-wetting fluid will invade pore spaces and displace the wetting fluid. The relationship between capillary pressure and wettability, as determined through contact angle, can be explained mathematically using the Laplace equation:

$$
P_{c}=P_{n w}-P_{w}=\frac{2 \sigma \cos \theta}{r}
$$

where $\mathrm{P}_{\mathrm{c}}$ is the capillary pressure $(\mathrm{Pa}), \mathrm{P}_{\mathrm{nw}}$ is the pressure of the non-wetting phase $(\mathrm{Pa})$, $\mathrm{P}_{\mathrm{w}}$ is the pressure of the wetting phase $(\mathrm{Pa}), \sigma$ is the interfacial tension between two fluids $\left(\mathrm{N} \mathrm{m}^{-1}\right), \mathrm{r}$ is the radius of the pore $(\mathrm{m})$, and $\Theta$ is the contact angle. This equation illustrates that capillary pressure is the difference in hydrostatic pressures between the wetting and non-wetting phases. In addition, changes in wettability will change the capillary pressure required for a fluid to imbibe into a porous material.

Contact angles can be advancing, liquid is pushed along the surface which creates the largest contact angle, or receding, liquid is pushed back. The difference between these angles is known as contact-angle hysteresis. This contact angle hysteresis is one of the causes of capillary pressure hysteresis and the effects can be seen when the two basic types of capillary pressure, drainage and imbibition, are examined (Anderson 1987a). 
Drainage is the increase in saturation by a non-wetting phase and imbibition is the increase in saturation of the wetting fluid (Anderson 1987a; Abdallah et al. 2007).

An understanding of capillary pressure-saturation $\left(\mathrm{P}_{\mathrm{c}}-\mathrm{S}\right)$ relationships (Figure 2) is necessary in multiphase models as they can be used to predict the distribution of fluid in the subsurface. When capillary pressure-saturation curves are prepared, the initial conditions include a capillary pressure of zero and an initial water (wetting fluid) saturation of 100 percent $\left(S_{w}=1\right)$. Capillary pressure is obtained by subtracting the pressure of the wetting fluid from the pressure of the non-wetting fluid. Drainage pressure is measured first by slowly increasing the capillary pressure which decreases the level of saturation in the sample. Eventually, irreducible wetting saturation is reached $\left(\mathrm{S}_{\mathrm{w}}=\mathrm{S}_{\mathrm{wr}}\right)$. At $\mathrm{S}_{\mathrm{wr}}$ any further increase in capillary pressure no longer affects the saturation level as the wetting phase is now only a thin film coating the soil grains and can no longer drain from the sample. This part of the curve is known as the primary drainage curve. At any point along this curve, the capillary pressure can be reduced; this is often done by decreasing the pressure of the non-wetting fluid, resulting in imbibition as the wetting fluid re-enters the sample. However, the wetting fluid saturation will never reach $100 \%$ as some of the pore spaces will now be occupied with non-wetting fluid. To determine the irreducible non-wetting saturation, the capillary pressure can be further decreased into negative values. At this stage, the wetting phase is forced into the porous medium. As with the drainage curve, when the imbibition curve is vertical, the irreducible non-wetting saturation has been reached. Therefore, differences in the imbibition and drainage curves, including the negative capillary pressures necessary to 
achieve non-wetting irreducible saturation, can be used to assess the wettability of a given system (Powers and Tamblin 1995; Powers et al. 1996).

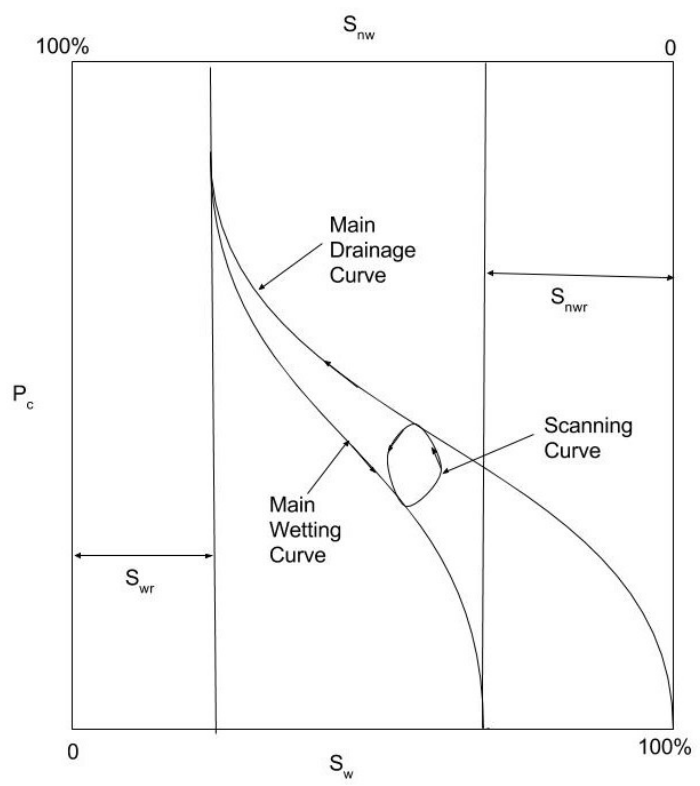

Figure 2: Capillary pressure-saturation curve, of a water-wet medium, showing effects of hysteresis; where $\mathrm{P}_{\mathrm{c}}$ is the capillary pressure, $\mathrm{S}_{\mathrm{w}}$ is the wetting fluid saturation, $\mathrm{S}_{\mathrm{nw}}$ is the non-wetting fluid saturation, $S_{\mathrm{wr}}$ is the irreducible wetting phase and $\mathrm{S}_{\mathrm{nwr}}$ is the residual non-wetting phase (adapted from Pankow and Cherry 1996).

Capillary pressure-saturation curves are obtained using empirical models (Brooks and Corey 1964; van Genuchten 1980). A major drawback of these models is that they are only valid when the capillary pressure is positive. However, when NAPL-wetting pores are present in the sample, the level of water saturation at any given capillary pressure decreases, effectively lowering the entire curve, and can result in forced imbibition for which the capillary pressure is negative. In order to account for the negative capillary readings, a simple transformation of the data can be used. For example, the original van Genuchten (1980) equation: 


$$
P_{c}=\frac{1}{\alpha}\left[\left(S_{w}\right)^{n /(1-n)}-1\right]^{1 / n}
$$

where $\alpha$ and $\mathrm{n}$ are fitting parameters, can be modified to account for negative capillary pressures (Bradford and Leij 1995):

$$
P_{c}+\tau=\frac{1}{\alpha}\left[\left(\overline{S_{w}}\right)^{n /(1-n)}-1\right]^{1 / n}
$$

where $\bar{S}$ is the effective saturation. The $\tau$ parameter is usually taken as the lowest $\mathrm{P}_{\mathrm{c}}$ to ensure that the left side of the equation is always greater than zero and is a function of the percentage of the porous media which is NAPL-wet (Figure 3).

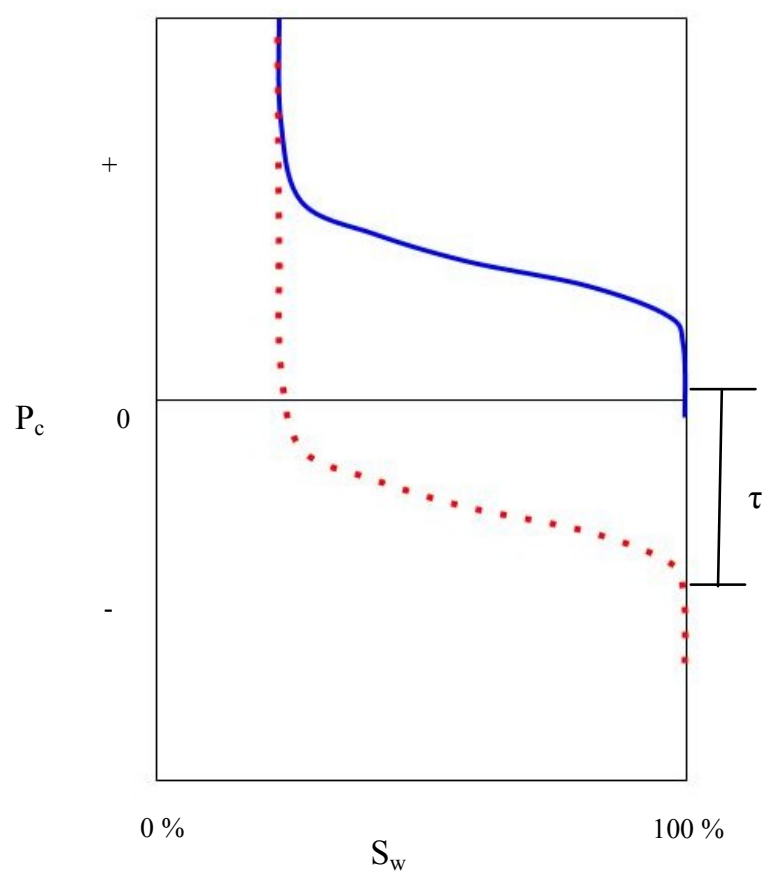

Figure 3: Capillary pressure-saturation drainage curve of a water-wet $(-)$ and NAPLwet ( $" . \cdot$ ) media. The $\tau$ symbol indicates the value needed to modify the van Genuchten equation per Bradford and Leij (1995). 
As previously stated, the wettability of the porous material will affect the behaviour of the pressure-saturation curves. Imbibition of water into a water-wet sample requires little energy; however, drainage, when water (the wetting fluid) is replaced by NAPL (the non-wetting) fluid, requires energy. The energy required is a function of the area under the capillary pressure-saturation curve. For the strongest water-wet materials, the residual and irreducible non-wetting saturations are indistinguishable. The opposite occurs when the sample is strongly NAPL-wet. In this case, the primary drainage curve requires a much lower capillary pressure to reach irreducible saturation and a significant level of energy is required for imbibition of the wetting fluid. This means that when a system is strongly wetting to a fluid, it is relatively easy for that fluid to spontaneously imbibe into the system. As the system approaches neutral conditions, the drainage and imbibition curves turn out to be more similar as the energy needed to fill the pores with either the wetting or non-wetting fluid approach one another (Anderson 1987a).

With research showing that the subsurface is often not strongly wetting to any fluid (Powers and Tamblin 1995; Powers et al. 1996), there has been a corresponding increase in studies examining the effects of fractional wettability on capillary pressure. There are two indices commonly used to quantify wettability of soils: USBM or Amott-Harvey (Figure 4). The USBM (U.S. Bureau of Mines) method uses the principal that more work is required to displace a wetting fluid than the non-wetting fluid. By determining the area under the $\mathrm{P}_{\mathrm{c}}-\mathrm{S}$ curve for the water (positive capillary pressure, $\mathrm{A}_{\mathrm{w}}$ ) and the second fluid (negative capillary pressure, $\mathrm{A}_{\mathrm{nw}}$ ), the USBM index can be calculated:

$$
I_{U S B M}=\log \left(\frac{A_{w}}{A_{n w}}\right)
$$


Therefore, the index is positive when the system is water-wet. However, Bradford and Leij (1995) found that the $\mathrm{I}_{\text {USBM }}$ can be positive even when a substantial fraction (up to $75 \%$ ) of the porous media is NAPL-wet. They believe that this result may be due to the experimental set-up as the measurements were taken over a narrow range of capillary pressures to ensure the equipment was not damaged. This resulted in relatively high residual saturations and may have introduced bias into the $\mathrm{I}_{\text {USBM }}$ calculations. In addition, the $\mathrm{I}_{\mathrm{USBM}}$ results and the fraction of hydrophobic sands in the sample produced a linear relationship. Therefore, the $\mathrm{I}_{\mathrm{USBM}}$ of a porous medium could be used to estimate the fraction of NAPL-wet material. The Amott-Harvey $\left(\mathrm{I}_{\mathrm{A}}\right)$ imbibition test compares the Amott water index $\left(\mathrm{I}_{\mathrm{w}}\right)$ and the Amott oil index $\left(\mathrm{I}_{\mathrm{o}}\right)$ :

$$
I_{A}=I_{w}-I_{o}
$$

These indices are ratios of the spontaneous imbibition to the total saturation change. An Amott-Harvey index of +1 indicates a strongly water-wet medium and an index of -1 for a strongly NAPL-wet medium (Abdallah et al. 1997). Both the USBM and the AmottHarvey method use the $\mathrm{P}_{\mathrm{c}}-\mathrm{S}$ relationship to evaluate the wettability of a porous medium, which is necessary, when attempting to ascertain the migration of NAPLs in the subsurface. 


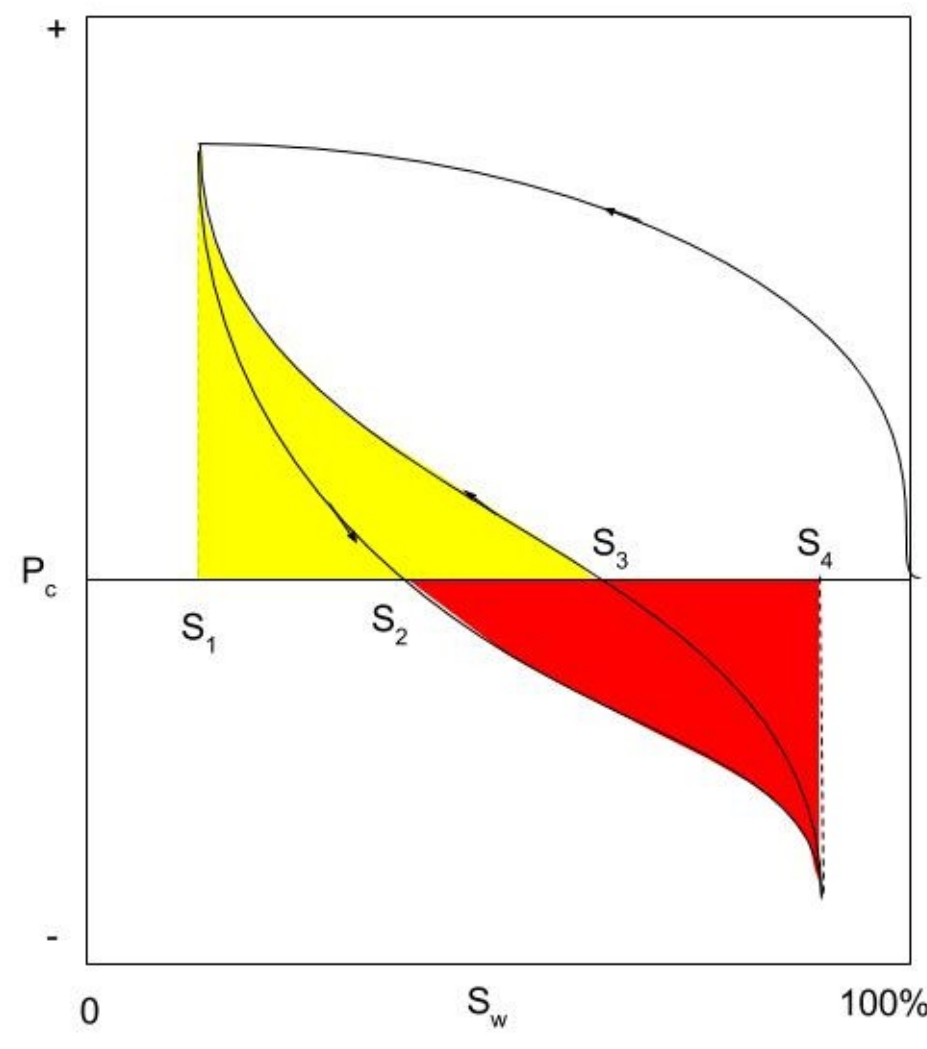

$$
I_{w}=\frac{S_{2}-S_{1}}{S_{4}-S_{1}}
$$$$
I_{o}=\frac{S_{4}-S_{3}}{S_{4}-S_{1}}
$$

$$
I_{A H}=I_{w}-I_{o}
$$$$
I_{U S B M}=\log \left(\frac{\square}{\square}\right)
$$

Figure 4: Methods to determine the USBM and Amott-Harvey wettability indices. The yellow region is the positive and the red is the negative areas of the capillary pressure saturation curves (adapted from Abdallah et al. 2007).

\subsubsection{Changes in wettability}

When the migration of NAPLs was first investigated, it had been assumed that all subsurface porous media were water-wet. However, although most porous media are naturally water-wet, the wettability of the soil grains can be altered. Factors which can affect wettability include: the composition of the subsurface, exposure to molecules and organics which sorb onto the solid surface, and exposure to surfactants and additives (Powers and Tamblin 1995; Anckner and Powers 1996; Powers et al. 1996; Harrold et al. 2001). Therefore, it is extremely important to know the saturation history of the material. 
Much of the work investigating NAPL spills uses laboratory grade solvents; however, the majority of NAPLs in the subsurface are combinations of many different chemicals and are likely to contain solutes as a result of their intended use (Pankow and Cherry 1996; Dou et al. 2008). These additions can include additives, surfactants, oils and greases.

As previously stated, NAPLs are a common contaminant found in groundwater and they can remain in the subsurface for long periods of time. Research has shown that exposure to certain NAPLs can alter the wettability of porous media. Powers et al. (1996) examined the effects of exposure to a variety of NAPLs on contact angle and capillary pressure-saturation curves. To determine if exposure to a NAPL would alter the wettability of quartz slides, the slides were left to equilibriate with the NAPL for seven days. The results indicate that a large range of wettability conditions could occur after NAPL spills. They found that the neat organic solvents had no significant effect on wettability, the petroleum products resulted in weakly water- to intermediate-wet conditions and the coal derived products resulted in NAPL-wet conditions. However, when capillary pressure-saturation curves were prepared, they did not clearly indicate the presence of NAPL-wet conditions even for those samples which resulted in NAPL-wet conditions according to changes in contact angle. The authors believe that this may be the consequence of mixed wetting conditions, a system containing both water- and NAPL-wet pores. Furthermore, the capillary pressure-saturation results are likely more indicative of actual subsurface conditions. When creosote first invades the saturated zone, the pores are likely to be water-wet; therefore, the creosote will invade the largest pores and is likely to only sorb to these pore surfaces. Some of the wettability changes observed in these experiments were unexpected, such as intermediate-wetting conditions 
that were found when the quartz slides were exposed to a gasoline/oil mixture obtained from the field. Based on the composition of these substances, prior to use, the quartz slides should have remained water-wetting. The authors have postulated three possible causes for the resulting wettability change: it may be the result of incomplete knowledge about the history of the site from which the sample was collected, it may also be due to the presence of other dissolved contaminants and finally the NAPL was in the subsurface for a period of time and had undergone weathering.

Anckner and Powers (1996) examined the changes in wettability of quartz surfaces when exposed to a variety of petroleum products over a 48 hour period. The chemicals tested included neat organic solvents and more complex commercially available NAPLs. They found that exposure to neat organic solvents had no significant effects on the wettability of the quartz and the surfaces remained strongly water-wet. However, some of the more complex NAPLs created surfaces which ranged from weakly water-wet to strongly NAPL-wet. Previous research had shown that asphaltenes and other medium to high molecular weight polar molecules were the major components in crude oil which could alter soil grain wettability (Powers et al. 1996). Anckner and Powers (1996) determined that although the higher weight molecules did alter the wettability of the quartz towards a more NAPL-wet surface, the surfaces exposed to gasoline also displayed a shift in their wetting characteristics from strongly water-wet to weakly waterwet. In this case, the change in wettability is believed to be due to the presence of polar species, particularly the addition of a surfactant.

Even very short term exposure, from 1 minute to 24 hours, to spent non-petroleum based NAPLs has also been shown to alter the wettability of soils. Harrold et al. (2001) 
performed experiments examining the changes to the wettability of sandstone and quartz slides exposed to DNAPLs recovered from the field, laboratory grade TCE and laboratory grade TCE mixed with two types of grease. The results indicate that exposure to field DNAPL had the greatest effect on wettability. After only one minute of exposure, the contact angle of the quartz slide changed from an average of $18.8^{\circ}$ on an untreated slide to an average of $112^{\circ}$, signifying a change from a strongly water-wet surface to a weakly NAPL-wet surface. The wetting behaviour did not change significantly with a longer exposure time. When exposed to the two synthetic waste samples, mixtures of TCE and grease, the changes in wettability were not as pronounced, ranging from a contact angle of $36.1^{\circ}$ to $51.7^{\circ}$ after 24 hours. These samples also exhibited a more gradual increase in contact angle with exposure time. This is in contrast to the exposure to laboratory grade TCE which made no difference in the contact angle until the slides were exposed for 24 hours, at which point the contact angle increased to $43.2^{\circ}$. The authors believe that the wetting changes may be the result of sorption of the NAPLs to the surface of the quartz slides. Furthermore, they state that the changes in wettability could be a result of the difference in IFTs of the samples. However, this is unlikely with these samples as the variations in the IFTs are at most $4.5 \mathrm{mN} \mathrm{m}^{-1}$. These results suggest that chlorinated solvents may have the ability to alter the wettability of porous material that they come into contact with even after short term exposure and that laboratory grade solvents can have effects on wettability although it is to a lesser degree and they require a longer exposure.

Surfactants have also been shown to alter wettability of porous material. The change in wettability is due to the interaction of the polar heads of the surfactant molecules with 
the pore surface. Work by Powers and Tamblin (1995) examined the effects of additives in gasoline on their ability to alter the wettability of quartz surfaces. Common additives in gasoline, including a surfactant, a corrosion inhibitor, a de-icer and a chemical used in deposition control, were mixed with pure-phase isooctane as the base. Results revealed that the surfactant (Dodecylamine - DDA) and the chemical used in deposition control (Polybuteneamine - PBA) had significant effects on contact angles, and thus wettability. These chemicals rendered the originally water-wet quartz slides intermediate-wet. When very high concentrations of the surfactant were used in the synthetic gasoline, it caused the slides to become NAPL-wet; however, the concentration required for this change is unlikely to occur in commercial gasolines. Imbibition tests, in which the porous medium is exposed to fluids and the wettability of the porous medium is determined by the phase which is most imbibed by the medium, were also performed and in these experiments only the addition of the surfactant resulted in a sample which presented a significantly different imbibition rate. The synthetic gasoline with the surfactant resulted in a sand which imbibed very little water, with less than $10 \%$ saturation after five hours. In contrast, the other synthetic gasoline samples did not produce results which were significantly different from the control case of quartz sand treated with isooctane alone. These results were surprising as the PBA contact angles suggested the porous media would be intermediate-wetting after exposure. The authors theorized that as PBA is a very insoluble chemical, the initial water saturation in the samples would significantly affect its ability to sorb onto the quartz surface. Further experiments seem to indicate that this hypothesis may be true as when the initial water saturation was decreased the final saturation level also decreased. 
Similar experiments were conducted by Harrold et al. (2005) in which the effects of additives in TCE on the wettability of quartz were studied. Four additives were examined: cetyltrimethylammonium bromide (CTAB), octadecyltrimethylammonium bromide (OTMAB), dodecylamine and octadecylamine. No changes in wettability were seen in the CTAB and OTMAB samples even after aging for 30 days. Dodecylamine was the only additive that had an effect on wettability with no aging, with a decrease in water saturation with increasing aging. With the octadecylamine samples, changes to wettability only occurred when the samples were aged. These researchers have shown that pure laboratory grade DNAPL is not likely to alter the wettability of porous media; however, spent DNAPLs have the potential to do so even in the short term. When postulating the movement of a DNAPL in the subsurface the potential for altered wettability should be addressed.

\subsection{Laboratory versus field dense non-aqueous phase liquids}

To date the majority of experiments which have examined the migration of NAPLs in the subsurface have used laboratory grade NAPLs that are likely to be dyed for ease of visual identification in flow cells (Oostrom et al. 2007). However, it has been shown that pure laboratory grade NAPLs can have very different properties than industrial NAPLs or spent NAPLs (Dwarakanath et al. 2002; Dou et al. 2008) and that the dyes used to

facilitate flow cell experiments can alter the properties of laboratory grade NAPLs (Jeong et al. 2002; Tuck et al. 2003).

Non-aqueous phase liquids are used for a variety of purposes including dry cleaning, degreasing and manufacturing. Each formulation of NAPL is specific to the job and to 
the supplier. Unlike a laboratory grade NAPL, industrial NAPLs are mixtures which can contain more than one NAPL, additives, surfactants, and if used, by-products of the job for which they were designed. It is these enhancements and extras which can alter the expected behaviour of a NAPL. Seo and McCray (2002) investigated multicomponent DNAPLs and developed a method to estimate the resulting interfacial tension. This method was a simple linear ideal mixing theory which uses the pure organic phase IFT of each component to estimate the resulting IFT:

$$
\sigma_{\text {mixture }}=\sum_{i=1}^{n} \sigma_{i} X_{i}^{N}
$$

where $\sigma_{\text {mixture }}$ is the IFT of the mixture, $\sigma_{\mathrm{i}}$ is the IFT of component $\mathrm{i}$ and $X_{i}^{N}$ is the mole fraction of component $i$. This method accurately predicted the IFT of mixtures of up to four laboratory grade DNAPLs with an average relative error of $9.3 \%$. Although this method was successful for combinations of pure laboratory grade NAPLs, it did not work for a spent DNAPL (Dou et al. 2008).

Dou et al. (2008) characterized a DNAPL recovered from a site which had formerly been used for the manufacture of nuclear materials. The area from which the samples were recovered was used as a hazardous material dumping site for several waste streams. A Gas Chromatography-Flame Ionization Detector analysis of the DNAPL indicated a sample which was $94.7 \%$ PCE and $4.7 \%$ TCE on a molar basis. This analysis also tentatively identified carboxylic derivatives and fuel hydrocarbons. When the samples were equilibrated with water, the $\mathrm{pH}$ dropped suggesting that the DNAPL contained some acid. An Inductively Coupled Plasma Mass Spectroscopy analysis disclosed that 18 metal species were present in the sample. Some of these species could be a result of the local mineralogy and/or groundwater while others are used in plutonium production 
or other processes which occurred on-site. In addition, the authors found that this DNAPL sample did not adhere to the linear IFT estimation method. In fact, the IFT of the field sample $(2.3 \pm 0.3 \mathrm{mN} / \mathrm{m})$, which was predominately PCE, was significantly lower than that of pure phase PCE $(44.2 \pm 0.4 \mathrm{mN} / \mathrm{m})$ or the expected IFT of the PCE/TCE mixture $(\approx 42 \mathrm{mN} / \mathrm{m})$ using the method of linear IFT estimation. These results suggest that minor constituents in DNAPL mixtures can significantly alter IFTs.

Dwarakanath et al. (2002) obtained samples of spent NAPLs from a variety of sources: degreasing solvents from two Air Force Bases in the U.S., a dry cleaning solvent from a U.S. Marine Corp Base, weathered jet fuel from a fire training area at an Air Force Base and weathered gasoline from a former refinery. They found that the interfacial tensions of these field NAPLs were between 8 and $18 \mathrm{mN} / \mathrm{m}$. These readings were significantly lower than those of comparable laboratory grade NAPLs. For example, the PCE rich DNAPL field samples had interfacial tensions of only 10.4 and $14.6 \mathrm{mN} / \mathrm{m}$; however, the interfacial tension of pure laboratory grade chlorinated solvents are usually between $30-45 \mathrm{mN} / \mathrm{m}$ (Dou et al. 2008). The authors suggest that the field DNAPLs may contain surfactants which can lower interfacial tension.

Harrold et al. (2003) examined the behaviour of lab grade PCE and TCE, a field DNAPL and industrial waste chlorinated solvent mixtures when they flow through a variety of unsaturated materials including organic rich and chalk samples. The results indicated that IFT is affected through contact with soil grains. During the majority of the experiments, the IFT of the DNAPL increased after flowing through unsaturated soil; however, the lab grade DNAPLs showed a decrease in IFT after contact with the organic rich soil sample. The increase in IFT for the lab grade DNAPLs in the non-organic rich 
soils is likely due to the removal of stabilizers (added by manufacturers to ensure the product remains stable) by adsorption to the porous material. This is similar to the results of the spent DNAPLs; however, in these cases, the increase in IFT is a result of the additives in the DNAPLs sorbing to the surface of the soil grains. In contrast, the decrease in the IFT of lab grade DNAPLs after contact with the organic rich soil is believed to be the result of the organic material desorbing from the soil grains and mixing into the solvent.

Other chemicals, including dyes, can affect IFT. Dyes are used to stain at least one fluid phase in visualization experiments and are generally assumed to have no effect on NAPL flow behaviour. Harrold et al. (2005) found that when TCE was mixed with octadecylamine, which has been shown to alter the wettability of quartz surfaces, the interfacial tension decreased. Tuck et al. (2003) examined the effects of a dye (Sudan IV) on the surface chemistry of laboratory grade NAPL. Most dyes used in NAPL research have Sudan III as the base molecule and the adding of methyl groups creates different types of dyes. The surface activity of these dyes is the result of the hydroxyl group and azo (nitrogen to double nitrogen) bonds. Results show that as the concentration of dye increased, the IFT and contact angle measured through the dyed fluid decreased. This also serves to decrease the entry pressure required to enter pore spaces. The authors felt that as the addition of dyes alters the flow behaviour of the NAPL, dyed NAPLs can serve as practical surrogates for field NAPLs. Jeong et al. (2002) examined the effects of dying PCE with Oil-red-O on the properties of remedial solutions. These experiments indicate that not only does the presence of the dye decrease the IFT of the PCE in water and ethanol, it can also increase the density and viscosity of 
the remedial solutions, which can decrease the effectiveness of the remediation. When any chemical is added to a DNAPL to be used in experiments, including the use of dyes for visualization, it should be documented and the potential alterations to the IFT of the mixture and wettability of the porous medium should be determined.

There have not been many studies on the effects of remediation techniques on field DNAPLs under laboratory conditions. Thakur et al. (1995) investigated the effects of using a field versus laboratory grade DNAPL on the effectiveness of waterflooding as a remediation technique. The field DNAPL was a complex mixture of chlorinated hydrocarbons, aromatic hydrocarbons, oils and dissolved plastic, with a density of approximately $1.3 \mathrm{~g} / \mathrm{cm}^{3}$. The laboratory grade DNAPL selected for comparison was 1,1,2 - trichloroethane (TCA), as its density, $1.44 \mathrm{~g} / \mathrm{cm}^{3}$, was similar to that of the waste sample. When first injected into saturated sand columns, the resulting waste DNAPL saturations were notably higher than those created using pure laboratory TCA. This meant that the waste NAPL was responsible for displacing more water from the columns. In addition, although the recovery efficiencies were similar for the two samples, the amount of water needed to displace the waste DNAPL (after 2.4 pore volumes waste was still being removed) was much greater than the amount required for the TCA (only an average of 1.0 pore volume). The authors believe that the differences in remediation were due to the effects of the significantly different viscosities of the two samples ( 0.119 $\mathrm{cP}$ for the TCA and $69.8 \mathrm{cP}$ for the waste sample) and the oily films which formed on glassware which suggested that the waste sample could have the potential to alter the wettability of porous media. Based on the viscosities, it was believed that the TCA would be removed in a uniform process as it was less viscous than the remediation fluid 
and that the waste DNAPL would be very difficult to remove. However, as previously stated, the recovery efficiencies were similar which the authors assume was likely a result of a change in wettability. Further experiments were conducted to confirm that the waste DNAPL could in fact lower the water-wetting preference of sandstone to near neutralwetting from water-wetting conditions. The authors concluded that the use of laboratory grade DNAPLs as a surrogate for field DNAPLs will introduce several errors into any model of flow behaviour.

\subsection{Remediation of non-aqueous phase liquids}

Groundwater contamination from non-aqueous phase liquids is a mounting concern. In fact, even with remediation, the majority of these contaminated sites do not meet clean-up criteria established by regulatory agencies (Pankow and Cherry 1996). As previously stated in this work, NAPLs can be problematic as they have low solubilities, low degradabilities, low interfacial tension and can be toxic at minute concentrations (Pankow and Cherry 1996). As a result of these properties, the remediation of a NAPL spill can be difficult.

Early work in NAPL remediation was simply an extension of the work conducted by petroleum engineers in an effort to increase oil production (Craig 1971). In these cases, the goal was to increase the profitability of oil wells, not complete removal (or remediation to drinking water standards) of the oil from the subsurface as is the case for many contaminated sites. There have been many techniques developed for the mitigation of NAPL contamination, including methods to contain the spill and/or remove the contamination. A common technique used in DNAPL remediation is mass-removal. 
Mass-removal technologies involve the injection of water, with or without chemicals, into the subsurface in an attempt to remove NAPL from the subsurface (Pankow and Cherry 1996). Two of the most common techniques are waterflooding and surfactant enhanced remediation (SEAR). Waterflooding involves the injection of water into the contaminated subsurface and its withdrawal from locations downstream. Surfactant enhanced remediation (also known as surfactant flushing) is similar to waterflooding; however, surface active agents (surfactants) are added to the injected water.

The greatest concern in remediation, other than reaching remediation goals, is the migration of NAPLs into previously uncontaminated areas. A method used in mass removal remediation to determine the potential for migration of NAPLs is the trapping number, $\mathrm{N}_{\mathrm{T}}$ (Pennell et al. 1996; Jin et al. 2007; Suchomel et al. 2007):

$$
\begin{aligned}
& N_{T}=\left|N_{C}+N_{B}\right| \\
& N_{C}=\frac{q_{w} \mu_{w}}{\sigma \cos \theta} \\
& N_{B}=\frac{\Delta \rho g \boldsymbol{k} k_{r w}}{\sigma \cos \theta}
\end{aligned}
$$

where $\mathrm{N}_{\mathrm{C}}$ is the capillary number, $\mathrm{N}_{\mathrm{B}}$ is the bond number, $\mathrm{q}_{\mathrm{w}}$ is the Darcy velocity of the aqueous phase, $\mu$ is the dynamic viscosity, $\sigma$ is the IFT, $\Theta$ is the contact angle, $\mathbf{k}$ is the intrinsic permeability and $\mathrm{k}_{\mathrm{r}}$ is the effective permeability. The larger the trapping number the more likely mobilization of the NAPL. In experiments conducted by Pennell et al. (1996), the critical trapping number for the removal of PCE appeared to be $2 * 10^{-5}$. Jin et al. (2006) found similar results in which DNAPL would not mobilize at trapping numbers less than $10^{-5}$, some mobilization occurred between $10^{-5}$ and $10^{-2}$ and complete mobilization occurred at any higher trapping number. 


\subsubsection{Waterflooding}

Waterflooding is one of the earliest remediation techniques used for clean-up of contaminated sites; the first instance of a waterflood was in 1865 to increase oil production (Craig 1971). This technique was particularly popular as water is generally readily available, the technology is easy to implement and use, usually water will freely move through a contaminated site and water is efficient at replacing oil (Craig 1971). Researchers quickly noted that for a successful waterflood, it was important to have a thorough understanding of the subsurface and the fluid properties. For example, heterogeneities in the soil will cause the injected water to move along preferential pathways of more permeable materials. Another factor which is known to strongly affect waterflooding efficiency is the viscosity ratio, $\mu_{\mathrm{nw}} / \mu_{\mathrm{w}}$ (Craig 1971). Due to factors such as these, there exists no specific optimum flood rate; it will be site specific. In fact, Oolman et al. (1995) found that an increase in the velocity of a waterflood did not significantly improve recovery efficiency.

The efficiency of a waterflood is known to be affected by the wettability of the porous media (Anderson 1987b; Dwarakanath et al. 2002). As previously discussed, the wettability of the subsurface can vary from water-wet to NAPL-wet conditions. These differences in wettability result in variances in flow and recovery of NAPLs from the subsurface. Waterfloods, under water-wet conditions, result in better recovery efficiencies. As conditions move to more NAPL-wet conditions, water will flow more easily through the system in comparison with the NAPL and result in lower recovery of the NAPL (Anderson 1987b). Under water-wetting conditions, when water is injected into the system, NAPLs are forced from small- and medium-sized pores into the center of 
the larger pores where the NAPL is more easily displaced. The expelled NAPL will move through the system along continuous channels. However, this also results in NAPLs remaining trapped as discontinuous blobs which are nearly impossible to remove with further waterflooding. In contrast, in NAPL-wet media water is trapped in the larger pores and the NAPL is found in smaller pores and as a thin film around the soil grains. When a waterflood occurs under these conditions, the water will flow along channels joining those larger pores and some water may invade and expel the NAPL from the smaller pores. However, the quantity of water required will be much greater and the recovery efficiency may never be as large as that under water-wet conditions. This was seen in work by Dwarakanath et al. (2002) through their examinations of the effects of wettability on the recovery DNAPLs from columns containing field soils which were remediated using a waterflood and then a surfactant flush. Waterflooding displaced more NAPL under water-wetting conditions than mixed- or NAPL-wetting conditions.

Oostrom et al. (1999a \& b) used a two-dimensional flow cell to determine possible explanations for the failure of a surfactant flush of a contaminated field site. As part of the experimental set-up, waterflooding and surfactant flushes were alternated. During the first waterflood the TCE which had accumulated on the medium-grained lens was removed or greatly reduced; however, the TCE which had pooled on the bottom finegrained layer was relatively untouched. The difference in removal was determined to be a result of the type of DNAPL, either entrapped or continuous. Entrapped DNAPL exists as isolated ganglia which provide greater surface area for dissolution and removal when compared with the continuous phase. Similar results were obtained by Fountain et al. (1991). In these experiments, waterfloods did not remove significant amounts of PCE 
from soil columns containing pools of PCE. Thus, although waterflooding can be used as a remediation technique, it is likely to only be suitable under specific conditions.

\subsubsection{Surfactant enhanced remediation}

Due to the low solubilities and high interfacial tensions of DNAPLs, waterflooding alone is usually not sufficient to achieve desirable levels of remediation. In order to improve the recovery efficiency of waterflooding, surface active agents, or surfactants, can be added to the injected water. This technique is known as surfactant enhanced remediation (SEAR). Each surfactant monomer is typically composed of a hydrophilic (polar) head and a hydrophobic (non-polar) tail. By having both hydrophilic and hydrophobic segments, surfactants are soluble to some extent in both polar and non-polar solvents (Lowe et al. 1998). Due to the dual nature of these molecules, they often accumulate with the hydrophobic tails facing each other, as they have very low solubility in water, and the hydrophilic head facing out towards the water (Lowe et al. 1998). When surfactants are used to remove contaminants, the tails will accumulate around nonpolar substances increasing their solubility due to the outward facing hydrophilic heads. In addition, surfactants are classified based on their hydrophilic group as either anionic (which are negatively charged) or nonionic (positively charged).

In an aqueous solution, surfactants will migrate towards any interface and orient themselves to minimize the contact between the hydrophobic phase and water. Surfactants work by altering the properties of the interfaces of solutions. They do so by changing surface tension, solubility and interfacial tension (Lowe et al. 1998). The 
changes in fluid properties can facilitate remediation through two methods: solubilisation and mobilization.

The orientation of the surfactant monomers will affect the method of remediation (Lowe et al. 1999). At high surfactant concentrations, the monomers form micelles. With the formation of micelles the remediation fluid results in a Winsor Type I system. These micelles are the accumulation of surfactant monomers around contaminant molecules. The threshold at which these micelles appear is known as the critical micelle concentration (CMC). At the CMC, the solubility of the NAPL begins to rapidly increase; surface and interfacial tensions are at a minimum. Any increase in surfactant concentration beyond that required to reach the $\mathrm{CMC}$ is incorporated into the formation of addition micelles. By creating units which are hydrophilic with a center which is hydrophobic, these micelles are solubilised and increase the dissolution of the NAPL. Therefore, during remediation under this system, NAPL is removed through partitioning into micelles, which are only found in the water phase. This results in a system containing two phases: the NAPL/water microemulsion phase (in which the NAPL is trapped within the micelles) and the excess NAPL. A Winsor Type I system is used when solubilisation of the NAPL is the major goal of remediation. When this system is used, cosolvents, such as alcohols or electrolytes, can be added to a surfactant flush to increase the solubility and effectiveness of the surfactant (Lowe et al. 1998).

As the concentration of surfactant is reduced, the solution transforms to a Winsor Type III system. In this system, three phases coexist: water, DNAPL and a surfactantrich middle phase. The middle phase results in a contaminant which is removed through solubilisation and mobilization. With a further reduction in surfactant concentration, the 
system will move towards micelles which form with the hydrophobic ends of the monomers pointing outwards, which is known as a Winsor Type II system. In this system, two phases exist: a DNAPL phase containing micelles filled with water and a water phase. Attempts must be made to avoid this system during remediation as the added surfactant is distributed into the NAPL with no increase in NAPL solubilisation or mobilization.

Mobilization arises due to the reduction in the interfacial tension between the NAPL and water. The reduction in the IFT will also diminish the effects of capillary forces which allows for greater migration of the NAPL. Surfactants decrease IFT by moving to the interface between two fluids and replacing original molecules. Many surfactants are available which can lower IFT; however, only certain surfactants are known to reduce IFT to levels conducive to mobilization of significant quantities of NAPL (Lowe et al. 1998).

There is one major undesirable side effect of mobilization, the uncontrolled downward migration of the NAPL (Fountain et al. 1991; Oostrom et al. 1999b; Jin et al. 2007). This will drive the NAPL deeper into the subsurface and there exists the possibility that previously uncontaminated areas may become so. To estimate the potential for uncontrolled downward migration, Jin et al. (2007) identified the dip angle $(\gamma)$, which is the angle which mobilized DNAPL will make with the $\mathrm{x}$-axis:

$$
\gamma=\arctan \left(\frac{N_{B}}{N_{C}}\right)
$$

Using this equation, to prevent unwanted downward mobilization of the DNAPL the lateral viscous forces must be greater than the buoyancy force. Under mobilization conditions, the direction of flow will be influenced by the dip angle which can allow the 
flow direction to be further subdivided into low downward risk (small gamma $<45^{\circ}$ ), transition zone and high downward risk (gamma $>70^{\circ}$ ). Unwanted downward mobilization was seen in experiments by Oostrom et al. (1999b). A two-dimensional flow cell was used to determine possible explanations for the failure of a surfactant flush of a contaminated field site. When surfactant flushes were utilized, the TCE dissolved more readily into the aqueous phase and created a plume which partially sank into the fine-grained bottom layer in unstable patterns. At the end of the experiment, it was determined that only approximately $60 \%$ of the TCE was removed. Part of the remaining TCE had migrated into the fine-grained bottom layer, which did not occur during the preceding waterflood.

Suchomel et al. (2007) examined the differences in recovery efficiencies of two surfactants: Tween 80 , which acts as a solubilising agent, and Aerosol MA, which acts as both a solubilising and mobilization agent. After seven pore volumes of Tween 80 were flushed through a contaminated 2-D box almost $90 \%$ of the TCE was removed. However, when only two pore volumes were used, the recovery efficiency was reduced to only $66.2 \%$ when the flow rate was maintained and removal efficiency was $83.1 \%$ when the flow rate was reduced. The reduction in flow rate increased the contact time between the surfactant and the TCE increasing the rate of solubilisation. In contrast with the Tween 80 findings, a higher flow rate with Aerosol MA resulted in a greater recovery, $81.3 \%$ versus $56.3 \%$ for the lower flow rate. The differences in removal efficiencies is thought to be the result of TCE penetration into a fine grained confining layer at the bottom of the box. This was expected as the total trapping number for this experiment was relatively high and the dip angle was likewise high. Surfactants can 
increase the recovery of NAPLs from contaminated areas; however, due to the possibility of unwanted migration of the NAPL, an appropriate surfactant must be carefully selected and the hydrogeology of the area characterized.

\subsubsection{Remediation of field non-aqueous phase liquids}

Although there have been many experiments to examine recovery efficiencies for a number of remediation technologies, the majority use only laboratory grade material (Oostrom et al. 2007). As previously stated, field NAPL and field soils are likely to have significantly different properties than laboratory grade materials. Due to these differences, techniques which work adeptly in the lab do not necessarily translate to a successful remediation in the field (Fountain et al. 1991; Oostrom 1999a \& b).

In studies, in which recovery efficiencies were studied under laboratory conditions using both laboratory and field materials, efficiencies between the two materials were quite different. Thakur et al. (1995) investigated the use of a laboratory grade DNAPL as a substitute for a field DNAPL in determining the effectiveness of a waterflooding scenario. The authors noted that although the experimental set-up was the same for each run, the recovery of the field DNAPL exhibited significantly more variation than the laboratory results. In fact, in the original experiments, the mobilization of the field DNAPL required a much greater pore volume to achieve any significant removal. The waterflood would be stopped at the end of the day and then restarted the following morning which resulted in a small slug of waste product when the experiment was restarted the following morning. To address this problem, the authors then preformed the experiments with continuous flow and the reproducibility improved. Other differences 
between the field and lab DNAPL include: original DNAPL saturations were greater for the field DNAPL, more water was required to achieve similar levels of DNAPL recovery for the field material and the TCA was displaced as a plug while the field DNAPL was only partially displaced as a plug. These results indicate that laboratory grade DNAPLs are not suitable alternatives to simulate field DNAPLs.

Heterogeneities in the subsurface will also influence the effectiveness of a surfactant flood. In experiments by Taylor et al. (2001), the effects of subsurface heterogeneity on surfactant enhanced remediation were examined. When 2-D box experiments were conducted, a surfactant flush promptly removed entrapped PCE; however, the pools of PCE above the fine grained lenses were relatively untouched through the surfactant flood. These results were similar to those obtained by others (Dekker and Abriola 2000; Conrad et al. 2002). The use of surfactants has been proven to increase the recovery efficiency of NAPLs. In fact, Childs et al. (2006) used a surfactant composed of Aerosol-MA, isopropanol and calcium chloride to achieve an overall PCE removal rate of $65 \%$ without unwanted migration. However, the use of surfactants can also cause complications and may not perform as well in the field as they do in the lab. Therefore, it is important to fully grasp the properties of the fluids and porous media present in the area of concern prior to the design of the surfactant flush. 


\subsection{Imaging analysis}

Ascertaining how NAPLs behave in the subsurface is difficult to accomplish in the field; therefore, scientists have created simulated environments into which contaminants could be spilled and be continuously monitored using non-invasive and non-intrusive techniques. Many types of laboratory techniques exist to both monitor and determine the level of saturation in porous media: gamma radiation, X-ray attenuation and methods using light (Oostrom et al. 2007). The most common methods used in image analysis are methods using light, as these methods are easy to use and the equipment required has the lowest associated costs (Niemet and Selker 2001).

When using image analysis methods that employ light, at least one side of the subsurface model is made of a transparent substance which allows the spill to be monitored and photographed. There are two methods which use light: light transmission and light reflection. In light transmission, both the front and the back of the subsurface model are transparent and the lights are placed at the back of the model allowing the light to shine through the model. This method is the most commonly used in quantitative and qualitative analysis (Oostrom et al. 2007); as the entire width of the model is illuminated, it provides a good representation of the entire model (Goswami et al. 2009). When using light reflection methods, only the front of the model is transparent and the lights are placed to illuminate the front of the model. This method is not as common (Oostrom et al. 2007), but it is useful when the porous media in the box are not translucent enough to allow enough light through for any type of useful image or if one side of the box is to contain instrumentation. One drawback of the light reflection method is the fact that the placement of the camera and lights means that the photos are only representative of what 
occurs at the front of the box and this raises the concern that the observed migration patterns may be the result of wall effects. To address this potential problem, van Geel and Sykes (1994) inserted pressure transducers in the back of a two-dimensional sandbox to confirm that an LNAPL had migrated to locations as it appeared from the front of the box.

Models using image analysis techniques can be sub-divided into three categories: pore-scale micro-models, one-dimensional column experiments and multidimensional aquifer scale models (Oostrom et al. 2007). Pore-scale micromodels are only useful in describing fluid movement at the pore scale as they only contain a few pores and are as wide as the typical pore space for the porous medium of interest. Column experiments are useful as they provide a preliminary introduction to the distribution of fluids in porous material. However, both pore-scale and column experiments are not characteristic of what would occur at the field scale. Therefore, to fully appreciate and properly model the distribution of any contaminant in the subsurface, a two- or three-dimension model is required.

These dimensional models had been used in oil engineering for a number of years. Fredich Schwille (1988) was among the first to use all three dimensional model categories to photograph what transpires when chlorinated hydrocarbons (CHCs), a type of dense non-aqueous phase liquid, are spilled in porous media. These experiments were then used in conjunction with physical models to outline the principles that regulate the movement of CHCs in the subsurface. Microscopic models were used to monitor the movement of both water and PCE through the pore spaces between glass beads. Column experiments were used to determine the infiltration velocities and retention capacity of 
TCE and PCE in a number of different sand packs of differing permeabilities. Twodimensional experiments were conducted in a large trough. The trough allowed for the monitoring of a PCE spill in a model aquifer with two layers of differing permeabilities. These experiments were used to monitor the effects of groundwater flow on the distribution of the dyed PCE. Like the work by Schwille (1988), the earliest NAPL flow experiments to use image analysis techniques were only qualitative in nature. These experiments would describe how the NAPL would flow in the subsurface given certain conditions with descriptions of the NAPL stating only if it was present in a location or not.

Qualitative techniques can only be used to generate a very broad idea of migration in the subsurface; quantitative techniques generate much more useful data. In 1981, Hoa was the first to use a light transmission visualization technique to quantify water saturation in a heterogeneous air-water-solid system. His method was based on knowledge that a ray of light will take one of three paths through the system: an empty pore (air filled pore), a fluid filled pore (saturated pore) or a partially saturated pore (airwater pore) and that the light intensity reaching an observer is a function of the level of saturation in each pore. This method is simple and easy to use; however, it does require a calibration curve between the level of saturation and light intensity.

By the 1990's, most image analysis involving the migration of NAPLs began to include quantitative as well as qualitative analysis (Oostrom et al. 2007). As the majority of NAPLs are clear in their pure phase, the majority of experiments using any type of light visualization techniques involve the addition of a dye to the NAPL or water phase (van Geel and Sykes 1994; Darnault et al. 1998; Conrad et al. 2002). The addition of a 
dye is necessary to distinguish the NAPL from water within the system. Even with the addition of a dye, early work in quantitative analysis could only differentiate large classes of concentration. For example, Schincariol and Schwartz (1990) could only differentiate areas of high tracer concentration, $50-100 \%$, and low concentration areas, $10-25 \%$. However, they later used the photographs obtained during that study to determine the dye intensity in each pixel. The pixel intensity was then converted into solute concentration (Schincariol et al. 1993). The methods used in this analysis were time consuming, as they used a gray scale in each photograph to convert transmittance into optical density, which was then linked to concentration. In order to focus on the NAPL, the authors subtracted a photo of the original tank (water and porous media only) from all subsequent photos; therefore, only the NAPL itself was left in the photographs. The use of background subtraction, the removal of the tank with only water and porous media from all subsequent photos, is a common technique in light visualization methods (Schincariol et al. 1993). This method is particularly useful as it helps to account for difference in lighting across the surface of the tank.

As the use of photography in image analysis is gaining popularity, it is important to understand what happens when a photo is taken. Although the basics of how images are recorded in both digital and film photography are very similar, the following explanation of how images are captured with a camera will focus on digital media as most of the cameras used in image analysis are digital providing both time and monetary savings.

When light arrives at a camera lens, it is incoherent; the rays arrive from every possible direction. The lens is used to focus the light and it is for this reason that lenses are the most important and expensive part of any camera; however, what we see as one 
camera lens is actually a series of simple lenses. The specific arrangement of lenses, predominantly the focusing element, is particular to the objective of the lens. Regardless of the lens used, as the light hits the lens, it is bent and the camera is designed so the beams will focus on an image sensor. To ensure the camera can focus without the photographer needing to move, a focusing ring is included within the barrel of the lens. The focusing ring allows the lens focusing element to be moved (White 2006). In a digital camera, these focused light beams are directed at an imaging array. These arrays are divided into two categories: charged coupled device (CCD) or complimentary metaloxide semiconductor (CMOS). Both of these types of sensors function in the same manner, although the CCD sensor is more sensitive. It can provide better images in dim lighting and, most importantly in image analysis, $\mathrm{CCD}$ cameras tend to produce images which contain less noise than CMOS cameras (King 2005). When these sensors are struck by light, they emit an electrical charge; the strength of which is determined by the amount of light. Photodiodes are used to measure light intensity; however, intensity alone does not produce a colour image. Therefore, in image sensors a combination of filters of three colours red, blue and green are placed before the photodiodes. The most common filter array is known as the Bayer array. This ensures that no two filters of the same colour are placed next to each other. A side effect of this type of array is that there are double the number of green filters compared with the blue and red. This is important as light in the green spectrum is closest to the middle of the visible spectrum and although green light is hardest for the photodiodes to register, it is also the colour which records more information than the other colours (White 2006). The number of pixels in a 
photo is a measure of the number of sensors. A higher number of pixels will provide an image with a higher resolution.

There have been a number of different uses of colour in quantification of a NAPL spill; however, they can be divided into two major categories: RGB and HSI. Both scenarios treat colour as a vector. In RGB, colour is a vector of three components: red, blue and green. HSI describes colour based on hue, saturation and intensity. Hue is the description of the colour, saturation is a measure of the dilution of the colour and intensity relates to the gray scale (Darnault et al. 1998). Van Geel and Sykes (1994) spilled n-heptane dyed with Sudan III and found that using a method based on the RGB scenario did not add any extra information to that obtained using black and white photos which only considered intensity. The LNAPL saturation was determined using a linear regression which was based on a grey scale value. These results were similar to those obtained by Darnault et al. (1998). In their experiments, Soltrol 220, an isoparaffine solvent, was spilled into dyed water. When the RBG vector was used to determine the water saturation, the colour yellow (which indicated that no water was present) had the same blue vector element as the blue colour which specified full saturation. Therefore, although to the naked eye the colours were significantly different, the RBG method was unable to adequately differentiate between colours in order to be useful in determining saturation levels. Instead, the hue of the colour was used in a linear regression to determine the water saturation levels. As colour itself is not a particularly useful mechanism in image analysis, photos are often converted into a greyscale prior to analysis. When this technique is used, each colour is equivalent to a certain intensity and the colours are easily distinguishable. 
Most image analysis techniques, regardless of the method of quantification used, follow a five step process. The first step is to gather data which is used for calibration; this consists of gathering images that contain a known property (such as saturation or concentration). These images are then processed to remove as many potential sources of error as possible, such as background substitution. In the third step, the processed images are then used to determine the relationship between some image property (usually intensity) and the known property. The actual physical experiments are then conducted and the images gathered during this time undergo the same processing techniques as were used for the calibration photos. After processing the photos, the calibration relationship is used to determine the property, e.g. saturation, which occurs during the experiments. The actual specifics of each step are particular to the experiments and method of data collection (Goswami et al. 2009).

Although image analysis techniques are a common method for quantification of saturations during simulated contaminant spills, they are prone to errors. One of the most common errors is non-uniformity in lighting (Goswami et al. 2009). If the lighting is uneven, it can result in errors in reading intensity at any given point and at any given time. The best ways to minimize these errors are to ensure that while each experiment is running the lighting is not altered and to use the background subtraction method. Errors may also occur with the images themselves if problems with the hardware or software arise. The set-ups used in image analysis are usually remotely controlled; this is done so the operator can arrange the experiment and then let it run with minimal input. One of the drawbacks of the remote system is that problems are not immediately noticed and problems may never be noticed if they correct themselves or are not apparent when the 
data collected is reviewed. Additional errors can occur with the calibration relationship; this is particularly problematic as calibration is mostly based on an approximate relationship (Goswami et al. 2009). If the calibration has any errors, all subsequent analysis will also be incorrect.

There have been attempts to both verify and quantify any errors associated with image analysis. In some cases, the image analysis results are compared with results obtained using other quantification techniques such as $\mathrm{x}$-ray attenuation or gamma radiation (Oostrom et al. 2007). Others have attempted to compare the results of the image analysis to a mass balance for the systems (Schincariol and Schwartz 1990). Another method involves a comparison of the dispersion coefficient, which is determined based on the image analysis, with previously published results (Niemet and Selker 2001). Each of these techniques have limitations. Goswami et al. (2009) found that using dispersion coefficients to quantify errors is not possible, although the results can be used to make qualitative statements. They also found that the mass balance method could not quantify errors either. In fact, with the mass balance method, a complete mass balance did not guarantee that the results were error free. The most significant problem with either method is that exact error is difficult to quantify as each data set should be compared with an analytical solution or exact physical measurements which are usually not available (Goswami et al. 2009). 


\subsection{Summary}

Non-aqueous phase liquid contamination is a widespread concern, as these contaminants can be toxic in minute quantities, can remain in the subsurface for long periods of time and are used in a wide variety of processes which can lead to their release to the environment. As such, considerable research has been done in efforts to improve remediation of NAPL contaminated sites. However, many of the remediation and modelling techniques which are tested successfully under laboratory conditions prove to perform poorly under field conditions. As clearly highlighted in the preceding review of the literature, there is a need to better understand how wettability, particularly intermediate wettability, and DNAPL composition impact the migration, distribution and remediation of DNAPLs under conditions that more accurately reflect real world conditions. This knowledge will result in improved remediation of sites contaminated with DNAPLs. In evaluating DNAPL spills in a variety of media using both pressure transducers and photographic evidence it is hoped that this research will provide a better understanding of the pressure changes within porous media, particularly in hydrophobic soils, and how that will affect both the migration patterns of the DNAPL and its subsequent remediation. 


\section{Materials and Methods}

In order to monitor the movement of a non-aqueous phase liquid in a porous medium, a thin two dimensional sand chamber was designed to allow for monitoring of DNAPL spills, both a laboratory grade dyed TCE and a DNAPL collected from the field, with each spill replicated. Pressure transducers were inserted into the back of the box to monitor both water and DNAPL pressure during the course of each experiment and photographs were taken to confirm the arrival of the DNAPL front and the corresponding pressure readings. The DNAPL was spilled into a variety of media, including: clean hydrophilic sand, clean hydrophilic sand which contained finer grained sand lenses and clean hydrophilic sand which contained hydrophobic sand lenses. The box was later subjected to a waterflood immediately followed by a surfactant flush.

\subsection{Sandbox}

In order to monitor the movement of a nonaqueous phase liquid in a porous medium, a thin two dimensional sand chamber was designed to allow for monitoring of NAPL spills. This chamber was largely based on the design used by Conrad et al. (2002) to visualize the effects of surfactant floods on DNAPLs. The box consists of a glass plate, $1.5 \mathrm{~cm}$ thick $\times 64 \mathrm{~cm}$ high $\times 64 \mathrm{~cm}$ wide, and stainless steel plate, of the same dimensions, separated by a $1 \mathrm{~cm}$ thick square aluminum spacer, composed of four separate rails: top, bottom and 2 side rails (Figure 5). A stainless steel plate forms the back of the box and can be equipped with up to 19 pressure transducers (Figures 6 and 7). When the chamber is assembled, the total external dimensions of the box are $4 \mathrm{~cm}$ thick $\mathrm{x}$ 
$70.4 \mathrm{~cm}$ wide $\mathrm{x} 64 \mathrm{~cm}$ high and the internal dimensions are $1 \mathrm{~cm}$ thick $\mathrm{x} 60 \mathrm{~cm}$ high $\mathrm{x} 60$ $\mathrm{cm}$ wide. The pane of glass and back plate were sealed to the spacer with the use of a Viton 1/8" O-ring embedded in channels in the spacer. These channels were $2 \mathrm{~mm}$ wide and placed $3 \mathrm{~mm}$ from the inside edge of the spacer. To ensure a proper seal between the spacer and the glass, U-shaped clamps were placed along the chamber sides, top and bottom.

To allow for vertical flow and gravity drainage, three 1/16" NPT holes were drilled in the bottom spacer. In order to ensure the media do not escape from the bottom, discs of 7 $\mathrm{mm}$ diameter of $200 \times 200$ corrosion-resistant phosphor bronze woven wire cloth were counter-sunk into the holes. For injection of the NAPL and to allow for vertical flow, three 1/16" NPT holes were drilled into the top section of the spacer. In both the top and bottom rails (Figure 8), the center hole is located in the center of the rail and other holes are placed $16.4 \mathrm{~cm}$ from either side of the center hole. Five 1/16" NPT holes were drilled in the two side rails of the spacer (Figure 9). To ensure an even horizontal flow could be achieved, manifolds, which were $6 \mathrm{~mm}$ wide and $58 \mathrm{~cm}$ long covered by $200 \times 200$ corrosion-resistant phosphor bronze woven wire cloth, were placed along the inside portion of each rail.

To obtain the desired flow conditions, fittings were used to allow water flow to the holes which were required and plugs were used to ensure that water would not escape from holes which are not in use for the experiment. The fittings used were Swagelok connectors (B-200-1-1) with 1/8" vinyl tubing (Swagelok LT-2-4).

The box is put together by first laying flat the back plate and placing the assembled spacer so the edges of the spacer and plates joined. Once properly positioned, the glass 
pane is placed on top of the spacer, again ensuring that the pane is centered and the $\mathrm{O}$ ring in the spacer is within the channel. At this stage, U-shaped clamps were attached tightly to the sides and bottom of the box to ensure a tight seal. The pressure transducers were then inserted into the back plate. Once the transducers were attached, the box was placed in an upright position and the top rail carefully removed to allow the box to be filled with the desired porous media. After the box was filled, the top rail was reattached, the top clamp affixed and all clamps tightened. The box can then be positioned for the experiments.

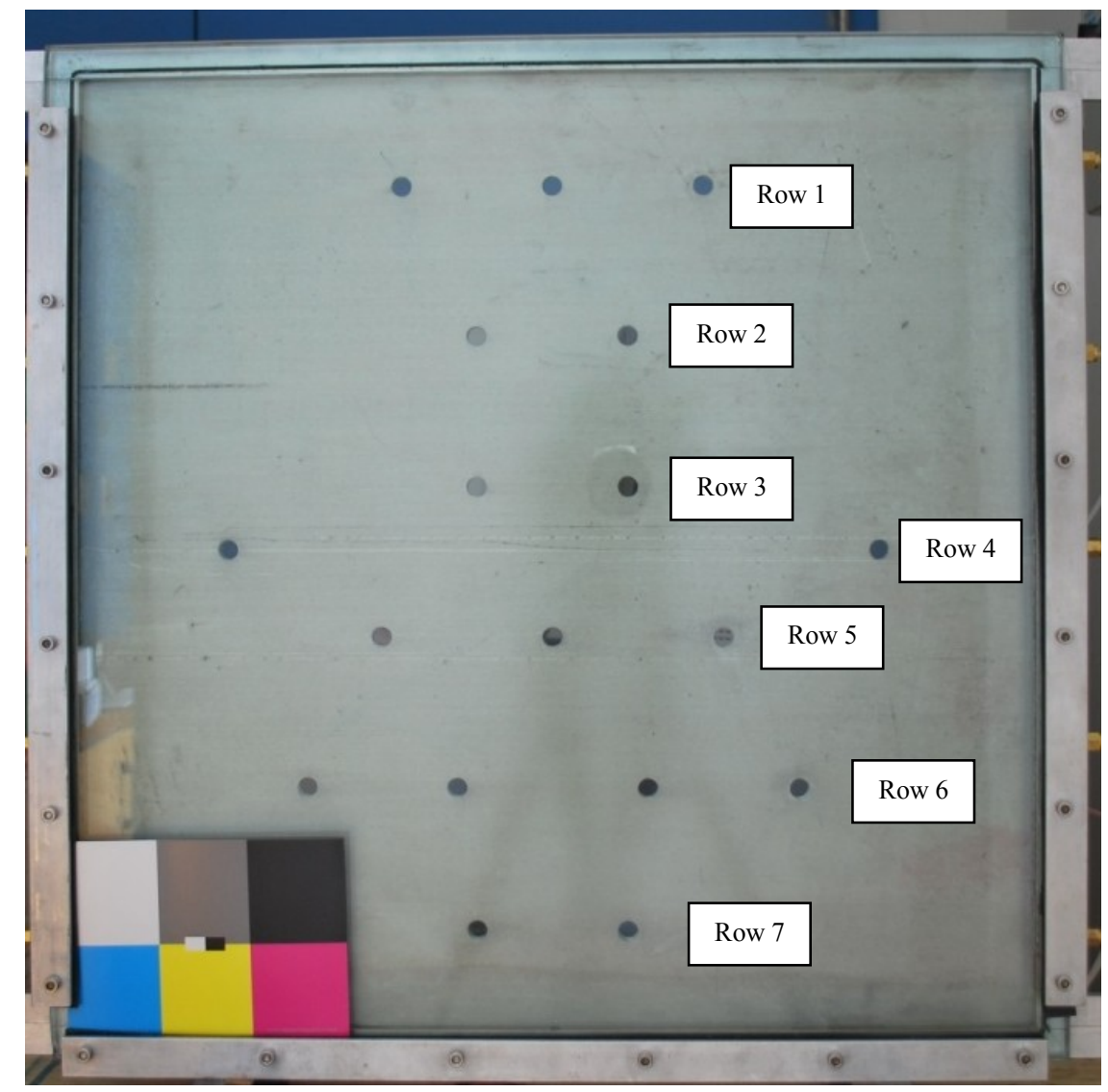

Figure 5: Assembled box, prior to placement of pressure transducers and porous media. 


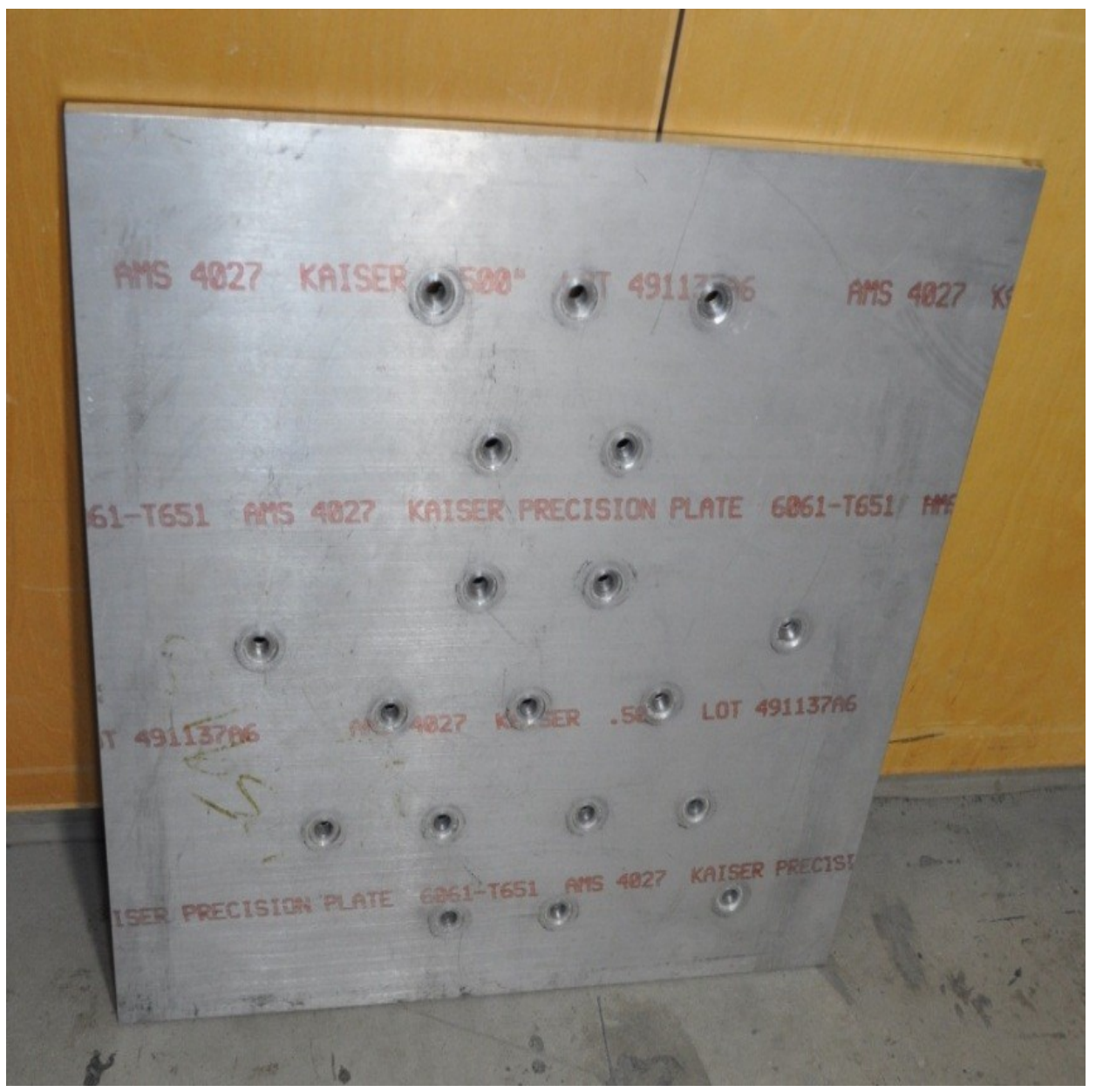

Figure 6: Back plate of sand box. 


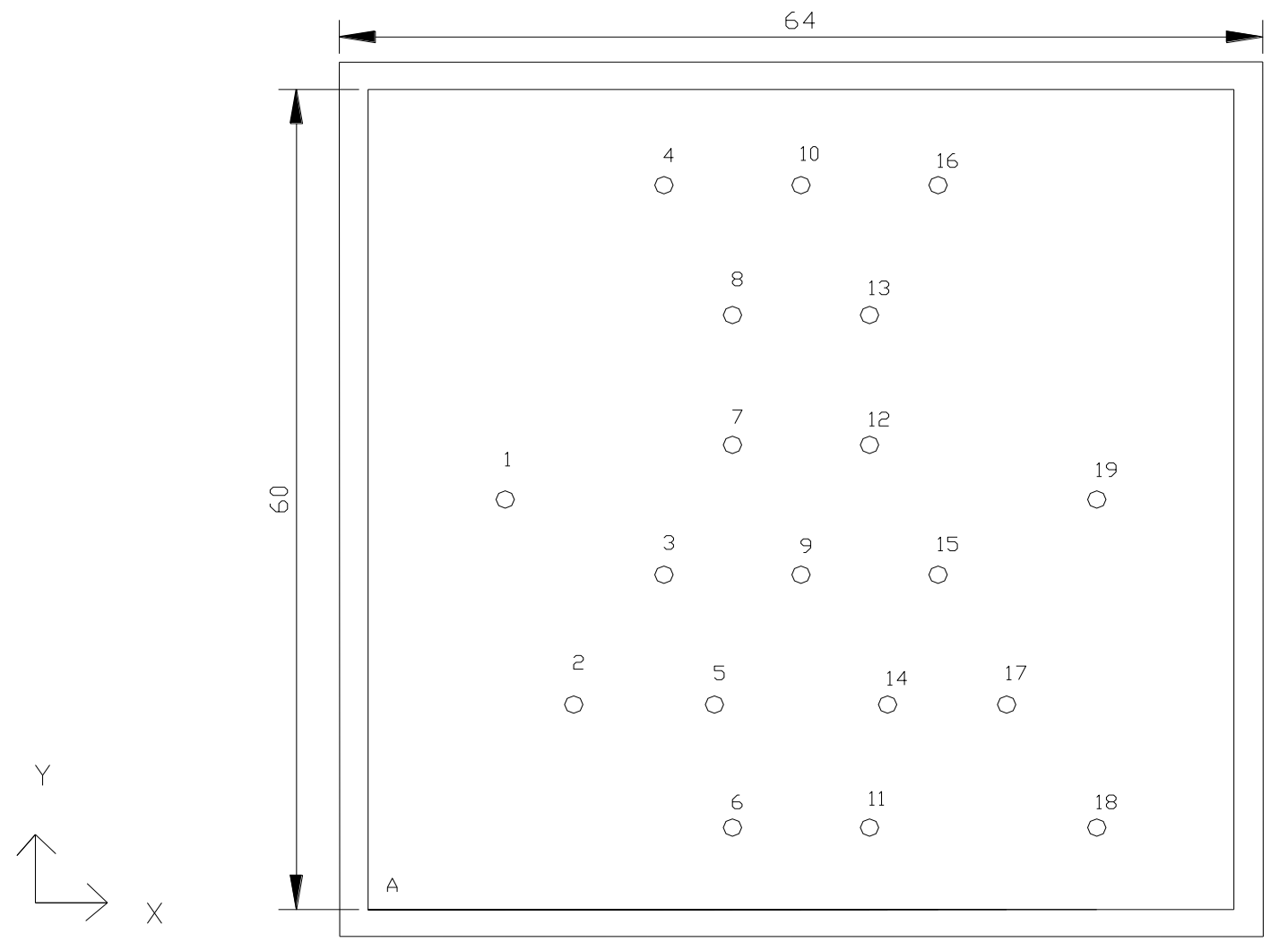

\begin{tabular}{|ccc|ccc|}
\hline Point & $\mathrm{X}(\mathrm{cm})$ & $\mathrm{Y}(\mathrm{cm})$ & Point & $\mathrm{X}(\mathrm{cm})$ & $\mathrm{Y}(\mathrm{cm})$ \\
\hline A & 0 & 0 & 10 & 30 & 53 \\
1 & 9.5 & 30 & 11 & 34.75 & 6 \\
2 & 14.25 & 15 & 12 & 34.75 & 34 \\
3 & 20.5 & 24.5 & 13 & 34.75 & 43.5 \\
4 & 20.5 & 54 & 14 & 36 & 15 \\
5 & 24 & 15 & 15 & 39.5 & 24.5 \\
6 & 25.25 & 6 & 16 & 39.5 & 53 \\
7 & 25.25 & 34 & 17 & 44.25 & 15 \\
8 & 25.25 & 43.5 & 18 & 50.5 & 6 \\
9 & 30 & 24.5 & 19 & 50.5 & 30 \\
\hline
\end{tabular}

Figure 7: Schematic of back plate for sandbox; all holes are 1/4" NPT. The back plate is $64 \mathrm{~cm} \mathrm{X} 64 \mathrm{~cm}$; however, when assemble the visible area is $60 \mathrm{~cm} \mathrm{X} 60 \mathrm{~cm}$. 

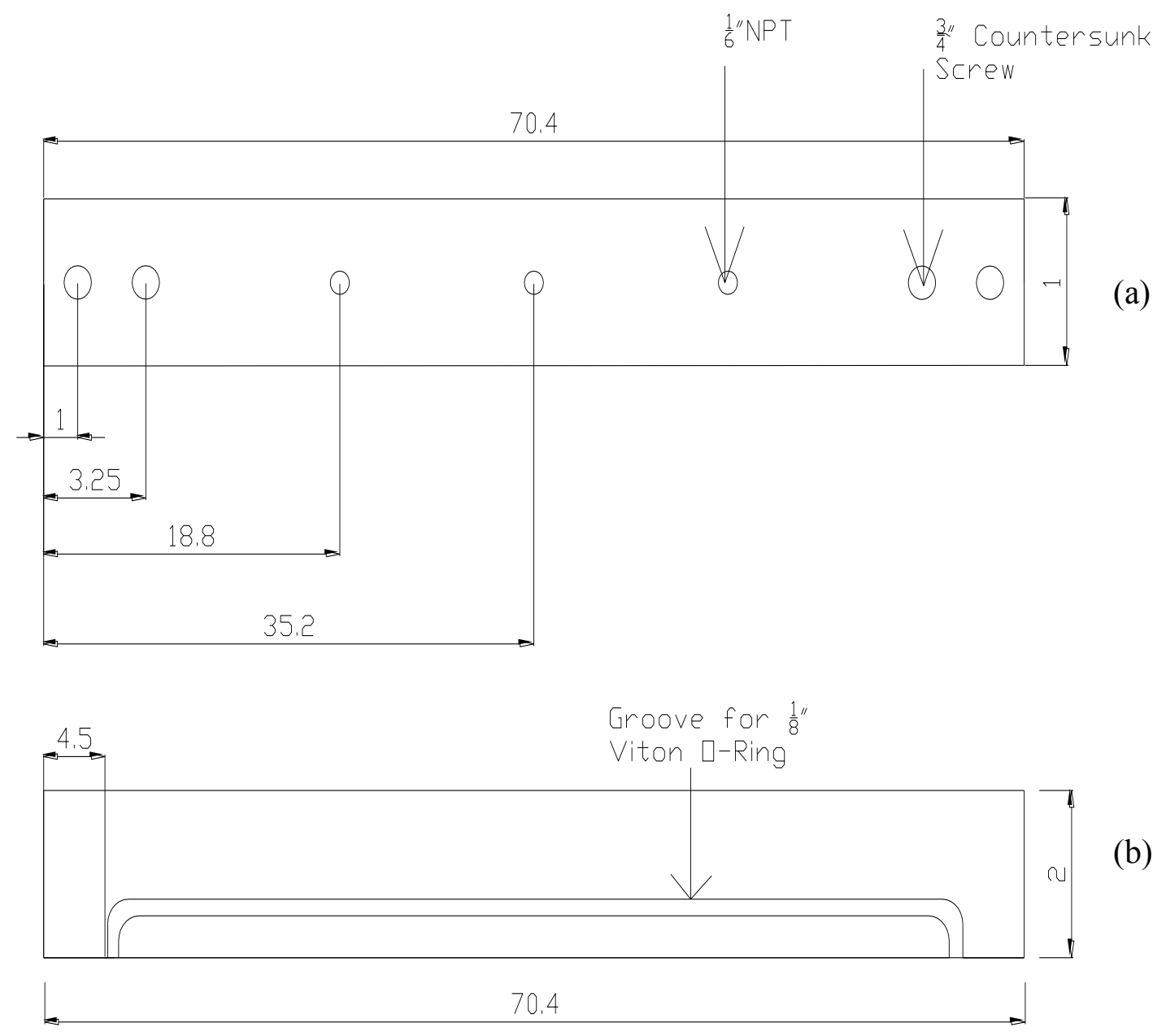

(b)

Figure 8: Schematic of top and bottom spacers; these spacers are identical, except for the placement of corrosion-resistant phosphor bronze woven wire in the $31 / 6$ " NPT holes in the bottom spacer to ensure no porous media escape the sand box; (a) is the top view of the spacer and (b) is the side view. All units in cm unless otherwise indicated. 


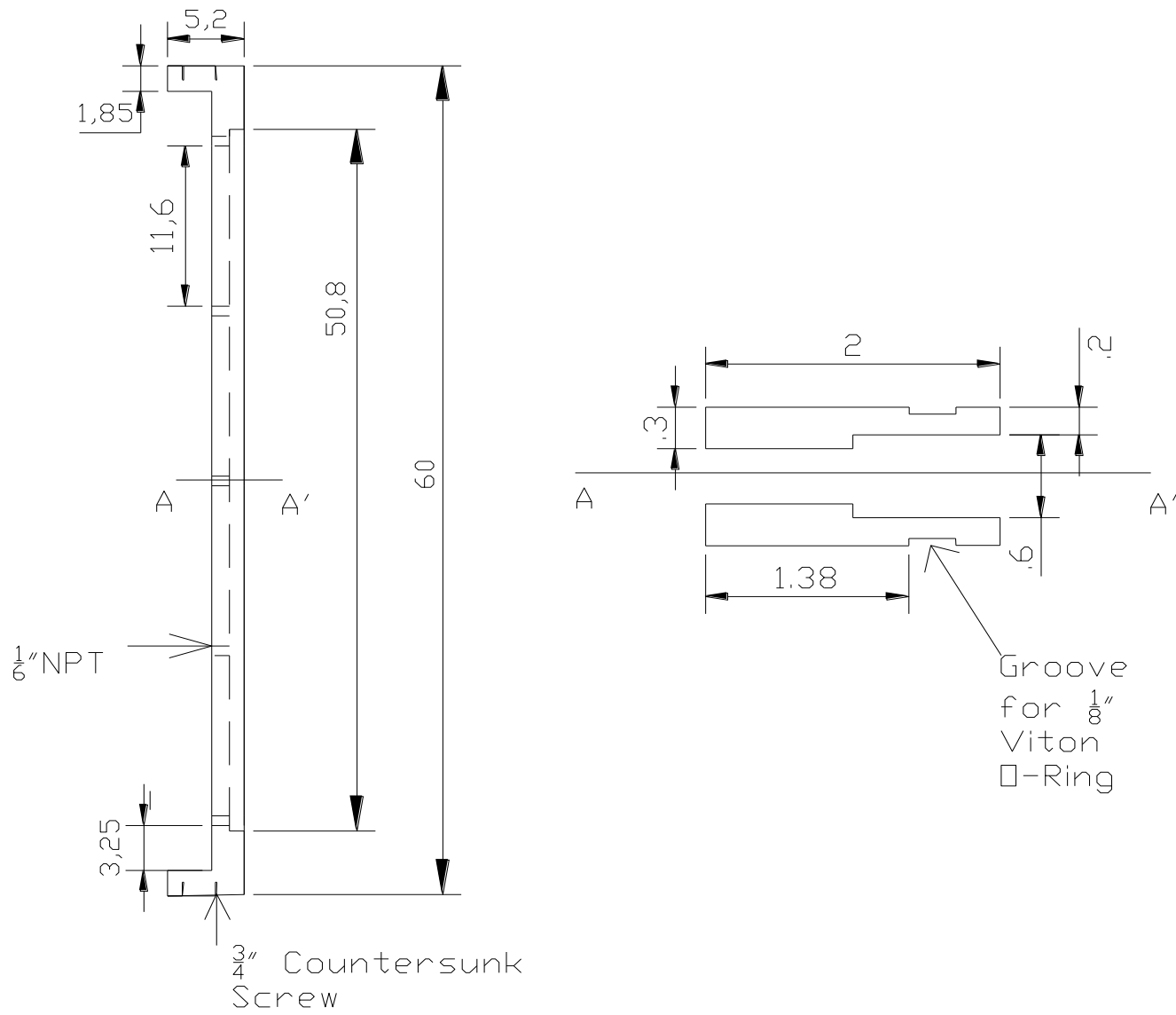

Figure 9: Schematic of side spacer used in the sand box construction. Units are in $\mathrm{cm}$ unless otherwise indicated.

\subsection{Pressure transducers}

The pressure transducers used in this work are a mix of 060-Model TJE with a range of -2.0 psig to $2.0 \mathrm{psig}$ and those with a range of -2.0 psig to $3.4 \mathrm{psig}$, both with an accuracy of $0.1 \%$ of full scale (Figure 10 ). Each transducer was filled with the fluid of choice, either water or the DNAPL of concern. This was done carefully to ensure that no air remained trapped within the cavity of the transducer. The transducers were attached to the back of the box with stainless steel Swagelok connectors (SS-400-1-4-OR and SS- 
4-TA-14ST). In order to measure the pressure within the box, the open end of the fittings within the box were sealed with membranes, either hydrophobic or hydrophilic dependent on the fluid in the cavity. These membranes were attached to the fitting through the application of Viton Fluoroelastomer-liquid, from Peaseal Technologies. Prior to attachment to a transducer, the fittings were slowly and carefully filled with the required fluid to ensure they were free of air. The pressure transducers were tested and calibrated prior to the experimental run and a random selection of transducers were selected to be tested at the end of the experimental run to ensure that they continue to meet specifications.

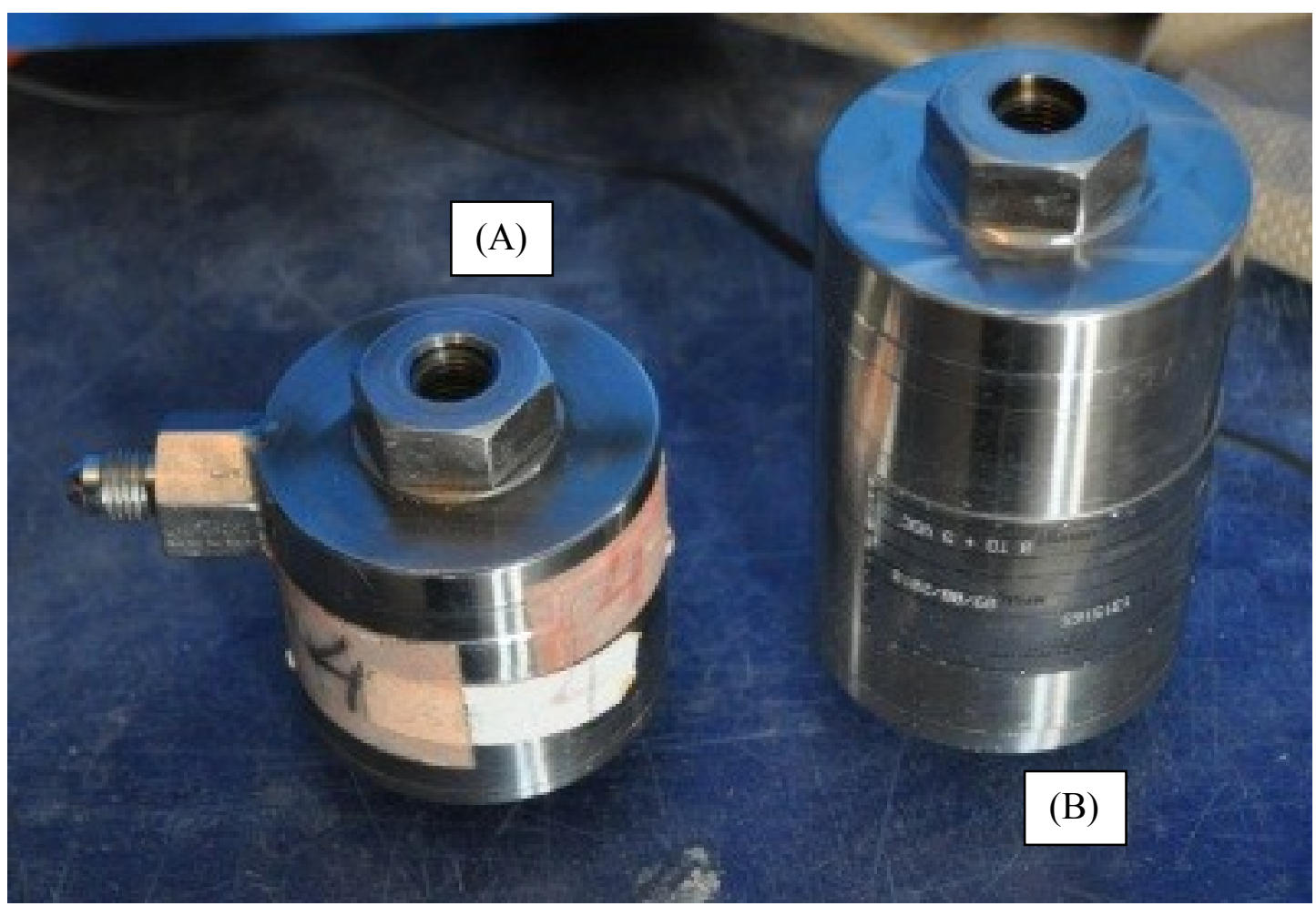

Figure 10: Pressure transducers used in experiments; (A) -2.0 to 2.0 psig and (B) -2.0 to 3.4 psig. 


\subsection{Nonaqueous phase liquids}

The nonaqueous phase liquids to be used in this investigation include: pure laboratory grade TCE and a field DNAPL. Table 1 lists some of the properties of the DNAPLs. The TCE was dyed with $0.05 \mathrm{~g} / \mathrm{L}$ Sudan III in order to allow for visualization of the spills. The field DNAPL was not dyed as it is already a dark colour.

Table 1: Properties of DNAPLs used in experiments.

\begin{tabular}{lcc}
\hline & TCE & Field DNAPL \\
\hline Density (g/mL) & 1.41 & 1.45 \\
Viscosity (cP) & $0.604(0.018)$ & $0.626(0.028)$ \\
Interfacial Tension (dynes/cm) & $29.79(0.12)$ & $26.78(1.88)$ \\
\hline
\end{tabular}

The DNAPL field sample was obtained from an industrial area in Ottawa, Ontario. There was no information available regarding the exact location of the contamination, site history, area geology or previous uses of the DNAPL. It is known that the sample was obtained from the bottom of a contaminated well. It was originally believed that this DNAPL was a spent DNAPL (had been used for the intended purpose). The field DNAPL appears as a dark, slightly-turbid sample. The DNAPL was characterized by Farah Hasson as a fourth year project in Chemistry at Carleton University. To characterize the DNAPL, it was first filtered to remove sand particles and the lightercoloured top most layer was disposed of. The actual characterization of the field DNAPL was conducted via a volatile organic compounds analysis (VOCs) by Paracel Laboratories (Ottawa, ON) using Gas Chromatography-Mass Spectrometry. The results of which specify the sample is $72 \%$ TCE, $27 \% 1,1,1$-TCA and approximately $1 \%$ PCE. Further testing by Paracel Labs determined that surfactants were not present in the 
sample; anionic MDL $<10 \mathrm{ppm}$ and nonionic MDL $<20 \mathrm{ppm}$. The density of the sample was determined gravimetrically and viscosity was measured using an Ostwald Viscometer. Interfacial tension was determined using an Axisymmetric Drop-Shape Analysis System. When the results of these tests were compared with the known density, viscosity and IFT of the pure laboratory grade sample, it suggests that although the sample was obtained from the field, it is unlikely that it had been used and was more likely the result of a spill or leak from a storage container of unused product.

\subsection{Surfactant selection}

The surfactant mixture used in these experiments was comprised of $4 \%$ Tween 80 (polyoxyethylensorbitan monooleate $-\mathrm{C}_{64} \mathrm{H}_{124} \mathrm{O}_{26}$ ), obtained from Sigma-Aldrich, and $0.5 \mathrm{~g} / \mathrm{L}$ calcium chloride $\left(\mathrm{CaCl}_{2}\right)$. Tween 80 is a nontoxic, nonionic surfactant and has been shown to highly solubilise TCE (weight solubilisation ratio of $1.47 \mathrm{~g} \mathrm{TCE} / \mathrm{g}$ surfactant) but does not result in a strong mobilization of TCE (Suchomel et al. 2007). It was chosen for this work as it is commonly used in both experimental and field surfactant flushes (e.g.: Taylor et al. 2001; Conrad et al. 2002; Franzetti et al. 2006; Suchomel et al. 2007).

Conrad et al. (2002) used a solution containing 5\% Tween 80 to achieve a recovery rate of almost $90 \%$ and no migration into lower permeability material for TCE dyed with Oil-Red-O spilled in a heterogeneous material. Taylor et al. (2001) used a mixture of tracer tests followed by a surfactant solution composed of $4 \%$ Tween 80 with calcium chloride added at a concentration of $0.5 \mathrm{~g} / \mathrm{L}$ to examine the effects of recovery of PCE 
from a porous medium with lenses of low permeability. They found that although the surfactant solution did well at removing the entrapped PCE, it did little to reduce the mobile phase pooled above the lenses of fine grained material. Suchomel et al. (2007) used a $4 \%$ Tween 80 solution to obtain TCE removal rates of 66 to $85 \%$. In addition, experimental work has shown that Tween 80 can have a high affinity for soil particles and high biodegradability (Franzetti et al. 2006).

The addition of the cosolvents, alcohols and electrolytes, increases the solubility and/or mobilization of the DNAPL when compared with the surfactant alone (Dwarakanath et al. 1999). Alcohols are added to surfactant mixtures in an effort to reduce the formation of gels, reduce equilibration times and reduce the viscosity of the resulting microemulsion. Electrolytes affect the salinity of the surfactant mixture and, based on their concentration, they can result in a Winsor Type I (water external microemulsion) or Type III (NAPL external microemulsion) phase behaviour.

\subsection{Porous material}

The porous media used in these experiments were C190 Accusand and C109 Accusand. The physical properties of the sands can be found in Table 2 .

Table 2: Properties of soil grains to be used in experiments.

\begin{tabular}{|l|c|c|}
\hline & C-190 & C-109 \\
\hline Median grain size, $\left.\mathbf{d}_{\mathbf{5 0}} \mathbf{( m m}\right)^{\mathbf{a}}$ & 0.73 & 0.37 \\
${\text { Uniformity index, } \mathbf{U}_{\mathbf{i}}^{\mathbf{a}}}_{\text {Porosity }^{\mathbf{b}}}$ & 1.21 & 1.95 \\
Permeability $_{\left(\mathbf{m}^{\mathbf{2}}\right)^{\mathbf{b}}}$ & 0.338 & 0.404 \\
\hline
\end{tabular}

a - Unimin, manufacturer of Accusand

$\mathrm{b}$ - O'Carroll et al. (2004) 
Sand is naturally hydrophilic; however, in many cases, a hydrophobic surface which is wetting to NAPLs is desired. A common method used to render porous media hydrophobic is treatment with 5\% (by volume) octadecyltrichlorosilane (OTS) in ethanol, after which the media is rinsed using ethanol. Work by Ahmed and van Geel (2009) has found that the use of this traditional method results in media which are hydrophobic but also contain excess OTS. The surplus OTS can be released to the surrounding hydrophilic sands rendering them hydrophobic as well.

Ahmed and van Geel (2009) developed a new method which significantly reduces the excess OTS in the treated sands. A solution of $5 \%$ (by volume) OTS ( $90+\%$, SigmaAldrich) in ethanol (Anhydrous Ethyl Alcohol) was prepared in a large sealable container. Enough solution was prepared to saturate the sample and completely cover the sand. The solution was stirred for three to five minutes after which the sand sample was added; the container was then tightly sealed and placed in a shaker. After 12 hours, the container was removed and the excess solution drained. The sand sample was then washed eight times with ethanol and air dried for 24 hours.

To ensure all excess OTS is removed, the sand was further washed using a Soxhlet extractor. The solvent used in this washing procedure was hexane (spectranalyzed 99.9\%, Fisher Scientific). The extractor was run for 12 hours after which the hexane used in the flushing procedure was examined and if cloudy, which indicates the presence of excess OTS, was then replaced with fresh solvent. After another 12 hour period (for a total of 24 hours), the thimble containing the sand was removed from the extractor and the sand sample allowed to air dry for 24 hours. When all the washings have been 
completed, the excess OTS on the sand will be significantly reduced (Ahmed and van Geel 2009).

\subsection{Experimental set-up}

A series of six experiments and a replicate of each were conducted, as follows, for a total of twelve experiments:

- Homogeneous water-wet sand (C-190) spill of dyed TCE

- Homogeneous water-wet sand (C-190) spill of field sample

- Heterogeneous water-wet sand (C-190 + lenses of C-109) spill of dyed TCE

- Heterogeneous water-wet sand (C-190 + lenses of C-109) spill of field sample

- Water-wet sand (C-190) with NAPL-wet lenses (C-190) - spill of dyed TCE

- Water-wet sand (C-190) with NAPL-wet lenses (C-190) - spill of field sample

After the box was assembled, it was filled with water. Once filled and examined

for any leaks, the box was filled with a series of lifts of sand, each approximately $1 \mathrm{~cm}$ deep, with agitation of the box occurring between each lift to ensure even distribution of the porous media. For the placement of the hydrophilic sand, each lift was poured as evenly as possible across the top of the box through a funnel (Figure 11) that extended from one side of the box to the other. To place the sand lenses (fine grained media and hydrophobic media), two semi-rigid pieces of plastic were inserted down through the funnel into the box and driven into the underlying layer of porous material (Figure 12). The hydrophobic sand was poured between the pieces of plastic in the funnel, in lifts of 1 $\mathrm{cm}$, until it reached a depth of approximately $4 \mathrm{~cm}$. Because the plastic spacers had to be inserted through the funnel, they were narrower that the width of the box chamber and 
some hydrophobic sand escaped between the plastic spacers and front and back plates causing the lenses to appear tapered, resulting in areas where the strongly hydrophobic and hydrophilic media were mixed. The pieces of plastic were left in place as hydrophilic sand was added on either side of the lens until the levels were equal. Then the pieces of plastic were carefully removed and lifts of hydrophilic sand continued until the next lens was added. During the placement of the sand, the top most holes in the side rails of the box were left open to allow for drainage of displaced water. Figure 12 is a schematic of the sand box with the placement of the lenses.
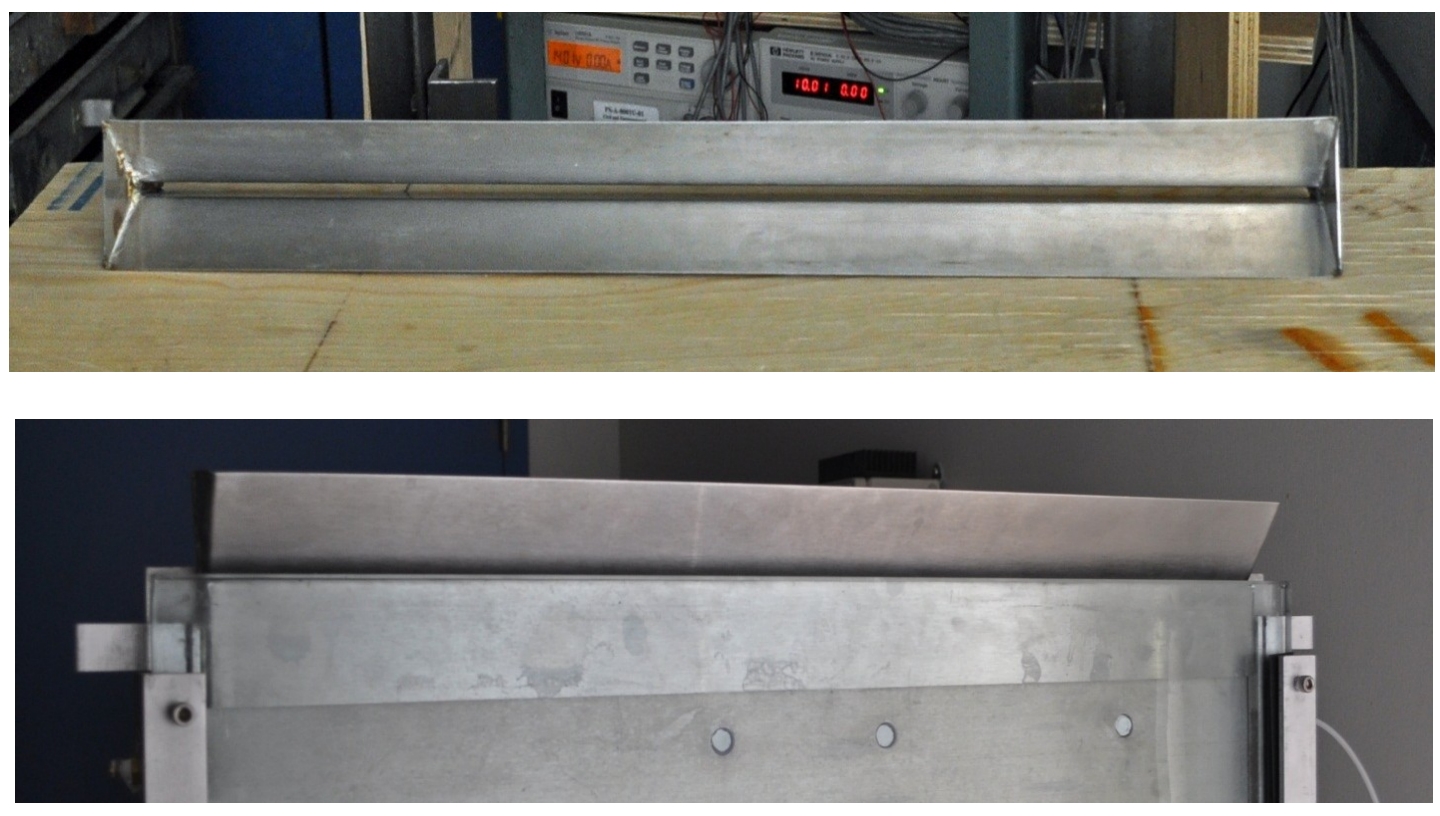

Figure 11: Funnel used to fill box with porous media. 


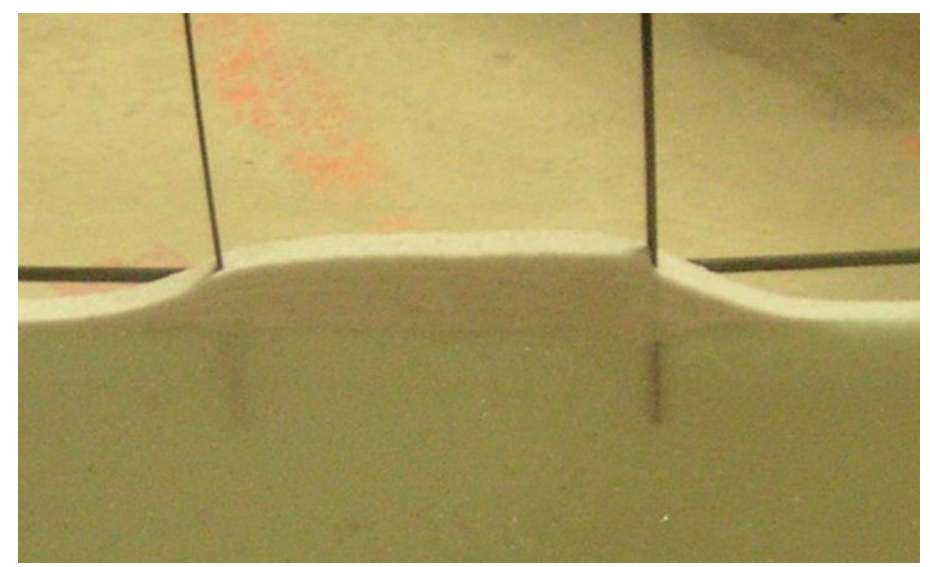

Figure 12: Placement of hydrophobic/heterogeneous lens; the stainless-steel back plate has been replaced by a pane of glass.

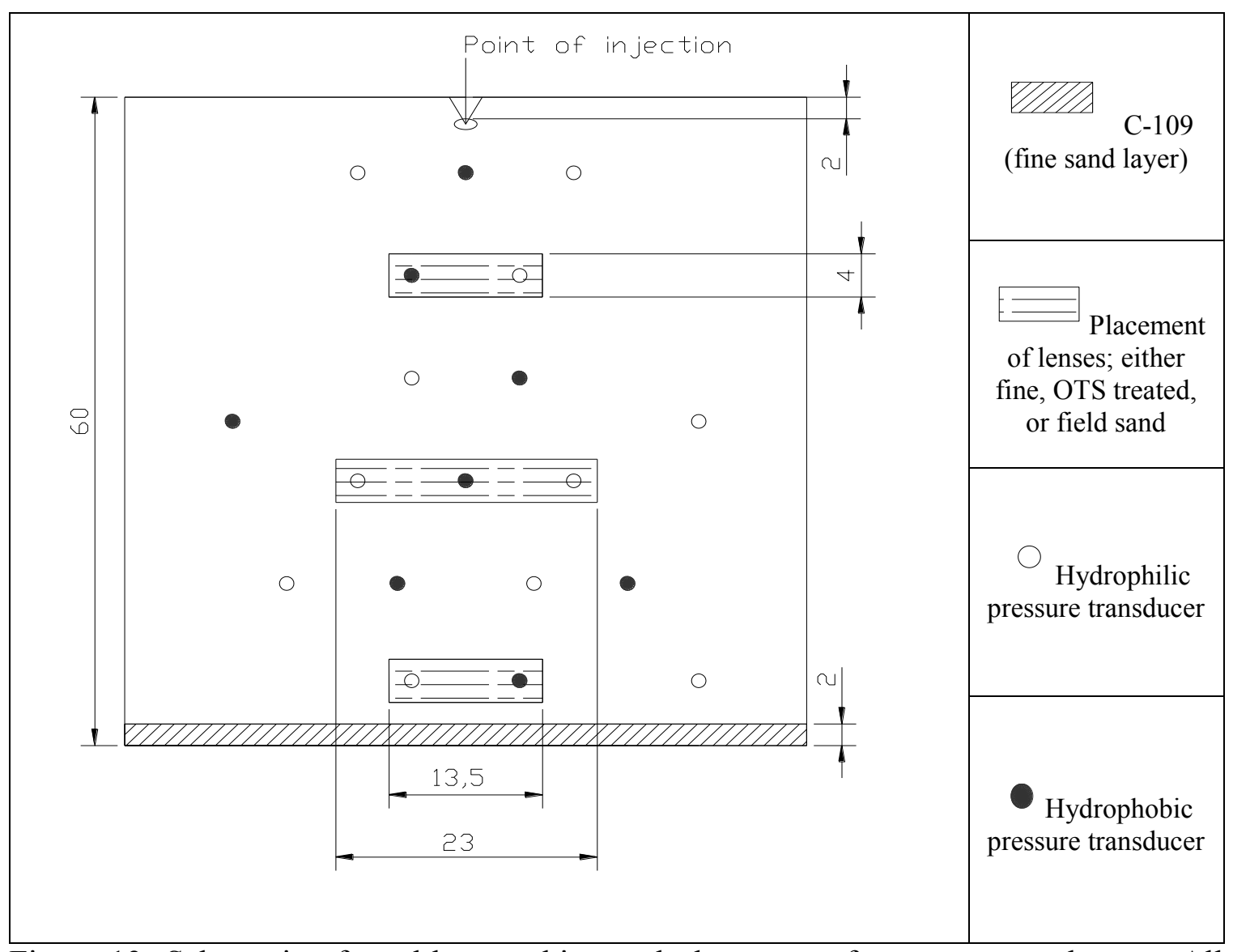

Figure 13: Schematic of sand-box packing and placement of pressure transducers. All units in $\mathrm{cm}$. 
When the box was completely filled with sand, the top spacer was replaced and the box sealed. It was then flushed with 4-5 pore volumes of water to maximize water saturation within the box. After the water flush, $75 \mathrm{~mL}$ of the DNAPL of choice was injected, using a syringe pump set to $0.5 \mathrm{~mL} / \mathrm{min}$. The spill occurred over a 2.5 hour period and the water displaced by the DNAPL was allowed to drain via the constant head wells on either side of the box. After the spill, the DNAPL was allowed to migrate and redistribute over a minimum 12 hour period after which a waterflood, at a rate of 5 $\mathrm{mL} / \mathrm{min}$, was initiated. This corresponds to a Darcy velocity of $0.08 \mathrm{~cm} / \mathrm{min}$ which reflects typical values for a waterflood or surfactant flush in the field (Conrad et al. 2002). For the waterflood and subsequent surfactant flush, the water entered the box through the vertical well on the left side of the box and drained via the vertical well on the right side. The waterflood was conducted for a minimum of 29 hours, which is equivalent to flushing 7 pore volumes through the box. Immediately following the conclusion of the waterflood, a surfactant flush at the same flow rate was started and continued for a further 29 hours minimum. The entire experiment was monitored using a camera to capture real time images of the movement of the DNAPL and pressure transducers were used to monitor the water and DNAPL pressures. To minimize reflection in the images captured by the camera, a white sheet was hung in front of the lighting system, consisting of a bank of three fluorescent lights, and the camera lens protruded through the sheet. 


\section{RESULTS}

Twelve spills in total were conducted; two spills each of the field and pure phase DNAPLs in three different porous media. There were no substantial differences noted between the spills with dyed laboratory grade TCE and the field DNAPL; in fact, there was as much variation between the replicate experiments as there was between experiments with the pure and lab grade DNAPL. Therefore, for each porous media type a representative spill was selected; based on both the photographic and pressure transducer data, which provided results typically seen in all four spills. In addition, the water pressures within the hydrophilic sand did not vary significantly throughout any of the experiments; therefore, the discussions will focus on the changes to DNAPL and capillary pressures. For every spill as the DNAPL was injected into the box, it began to pool at the surface of the saturated sand. The pool continued to increase until approximately $63 \mathrm{~mL}$ of the DNAPL had been spilled and the required entry pressure, corresponding to a pool height of approximately $2 \mathrm{~cm}$, was achieved. In all photographs of the box, the darker circle located approximately $16 \mathrm{~cm}$ below the top of the box is the reflection of the camera lens on the front of the box. 


\subsection{Homogeneous hydrophilic sand}

Figure 14 is an image of the box just prior to the commencement of the spill.

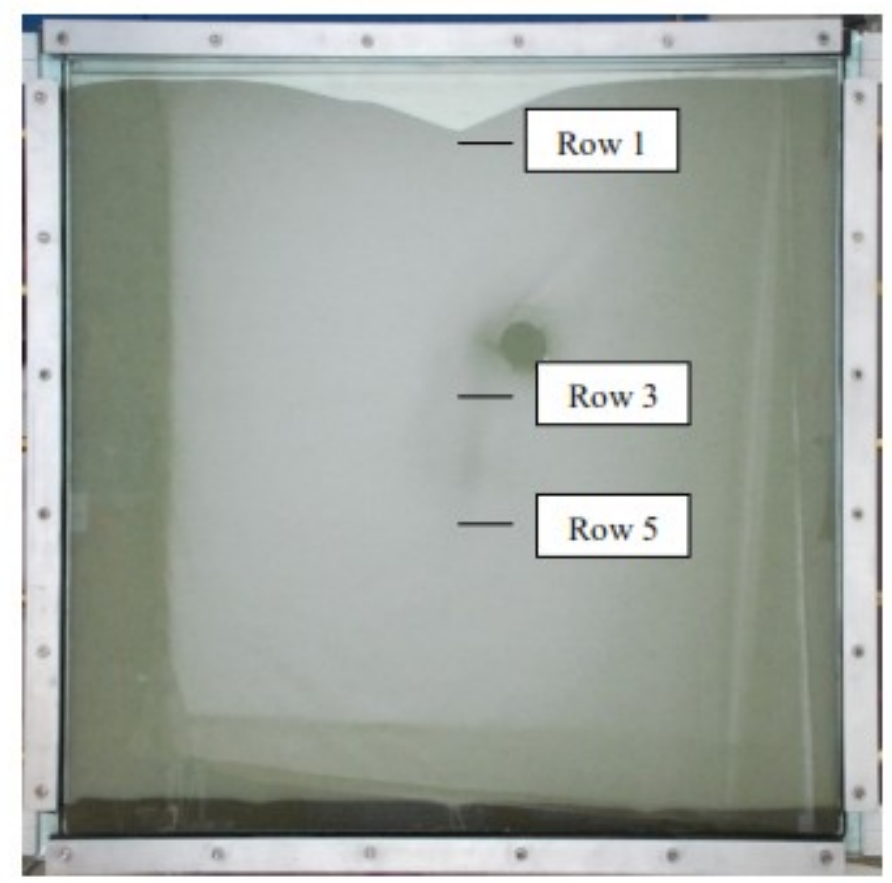

Figure 14: Image of the box prior to the spill in homogeneous hydrophilic sand, the black lines indicate the rows for which the pressure transducer data will be described.

After the entry pressure was achieved the DNAPL began its downward migration. This was accomplished as a thin front which was approximately $1.5 \mathrm{~cm}$ wide near the point of release and slowly spread laterally until it reached a maximum width of approximately $11 \mathrm{~cm}$ at the bottom of the box. When it came into contact with the layer of fine grained media at the bottom of the box, the DNAPL continued to spread laterally until it reached both edges of the box and continued to pool at the interface of the fine grained material. At the end of the spill, in most cases, there was a small pool of pure phase DNAPL at the interface of the water and porous medium at the top of the box. Some residual DNAPL formed a thin spill zone between the top and bottom of the box 
and the majority of the DNAPL as mobile phase pooled at the interface between the C190 sand and the fine grained media at the bottom of the box. The greatest concentration of DNAPL occurred where the spill zone came into contact with the fine grained material and the edges of the box (Figure 15). In addition, with no obvious cause when in contact with the outer edges at the bottom of the box the DNAPL appeared drawn upwards along those edges. The pool that formed at the bottom of the box varied depending on the trial - as it was highly dependent on the placement of the fine grained material which was never completely smooth. The pressure changes within the box for the homogeneous material were the most variable between trials when compared with the other porous media. This is because in some cases - due entirely due to the unpredictable sand grain placement - the DNAPL would come into contact with the pressure transducers.
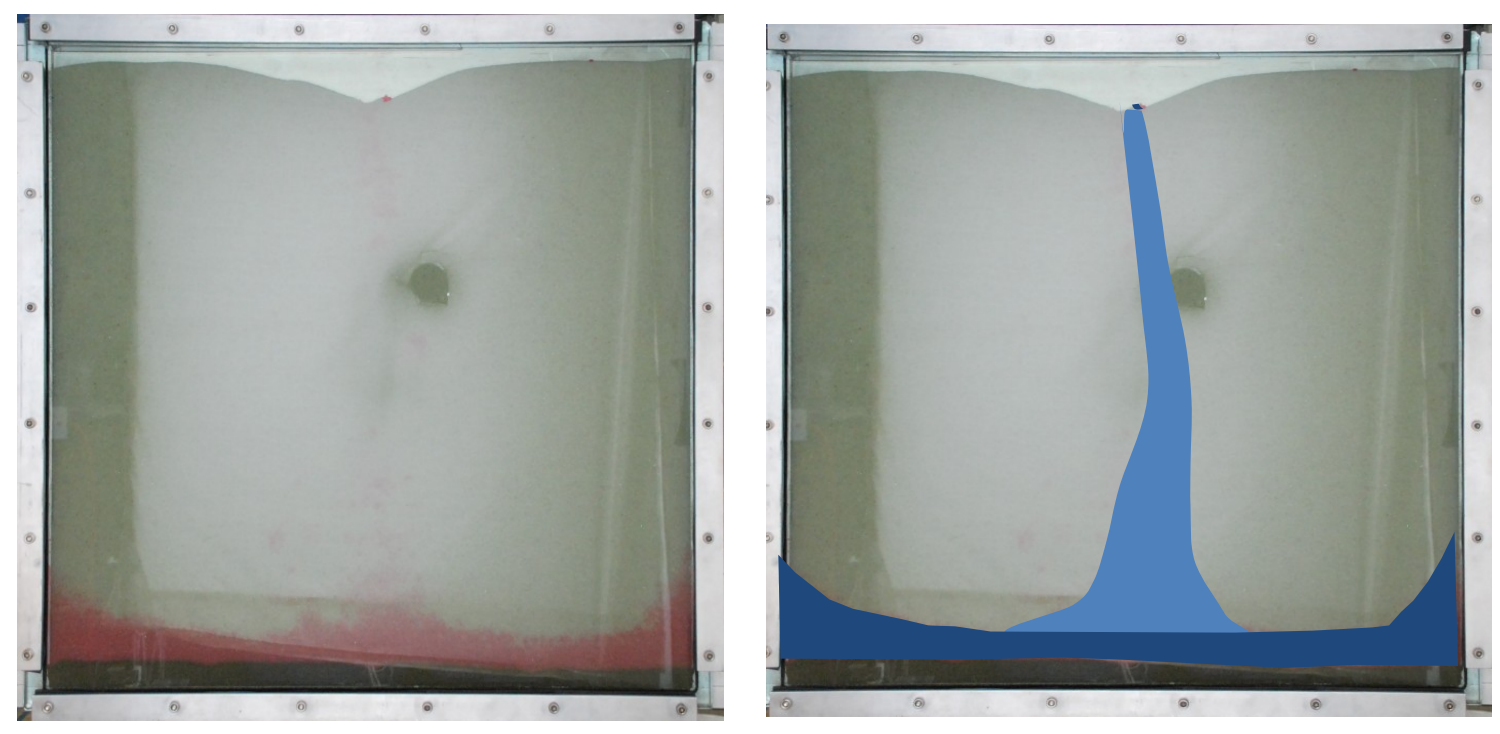

Figure 15: Position of the laboratory grade dyed TCE (red) after a spill (12 hrs). In the right hand photograph is a hand drawn interpretation of the DNAPL distribution. The dark blue areas are where DNAPL saturation is high and lighter blue areas indicates areas at which the DNAPL saturation is close to the residual value. 
In this particular case significant changes to the DNAPL pressure were seen in rows 2 (43.5 $\mathrm{cm}$ from bottom of box), 3 (34 $\mathrm{cm}$ from bottom of box,) and $5(24.5 \mathrm{~cm}$ from bottom of box). Row 1 (Figure 16A) shows the arrival of the DNAPL front approximately 1.5 hours after the start of the spill, once in contact with the transducer the increase in DNAPL pressure to its maximum was almost instantaneous. During the spill period, although the DNAPL pressure increased the resulting capillary pressure never rose above 0 , indicating an effective water saturation of $100 \%$. Surprisingly the DNAPL front came into contact with the transducers of row 3 (Figure 16B) earlier, approximately 0.5 hours after the start of the spill. This discrepancy is likely due to the slow lateral spread of the DNAPL front as the spill progressed. There was a spike in the DNAPL pressure when the front first arrived at the transducer but it quickly settle into a constant reading (approximately $43 \mathrm{~cm}$ of water pressure) for the remainder of the spill period. The effective water saturation in row 3 reached a minimum value of 0.02 , which is close to the maximum effective DNAPL saturation. However, after the spill ended (approx. 2.5 hours), the estimated capillary pressures do not indicate that the DNAPL drains from this location. The DNAPL front arrived at row 5 (Figure 16C) approximately 4 minutes after arriving at row 3 and the overall shape of the curves were similar to row 3 ; although the DNAPL did start to drain from the area prior to the end of the migration period. In addition, the effective water saturation in row 5 never got below 0.24 . It should be noted that at all three locations, the DNAPL reached the transducer locations causing the sharp increase in DNAPL pressures. It is unclear from the data whether just a film of DNAPL reached the transducers causing the sharp pressure change or whether a significant DNAPL saturation was reached at the transducer locations and the accuracy of the 
transducers impacted the estimated capillary pressure and corresponding DNAPL saturation. Overall, the pressure transducer data are supported by the photographic evidence; showing the arrival of the DNAPL to specific locations within the box during the spill at approximately the same time. However, it must be noted that the soils used in these experiments had very flat capillary pressure - saturation curves, the difference between $100 \%$ water saturation and the residual water saturation was less than $6 \mathrm{~cm}$. Therefore, even small changes to the capillary pressure could result in considerable changes to the level of saturation. The pressure transducers used in this study had an error range of $0.1 \%$ of full scale - which translates to an error of $+/-0.4 \mathrm{~cm}$ or $0.3 \mathrm{~cm}$ (depending on transducer type), additional errors include measurement error $(+/-0.5 \mathrm{~cm})$ during calibration of the transducers; resulting in a total pressure reading error of roughly $+/-0.9 \mathrm{~cm}$. This may be a reason for the inconsistency seen in some experiments between the photographic evidence and calculated saturation level. 

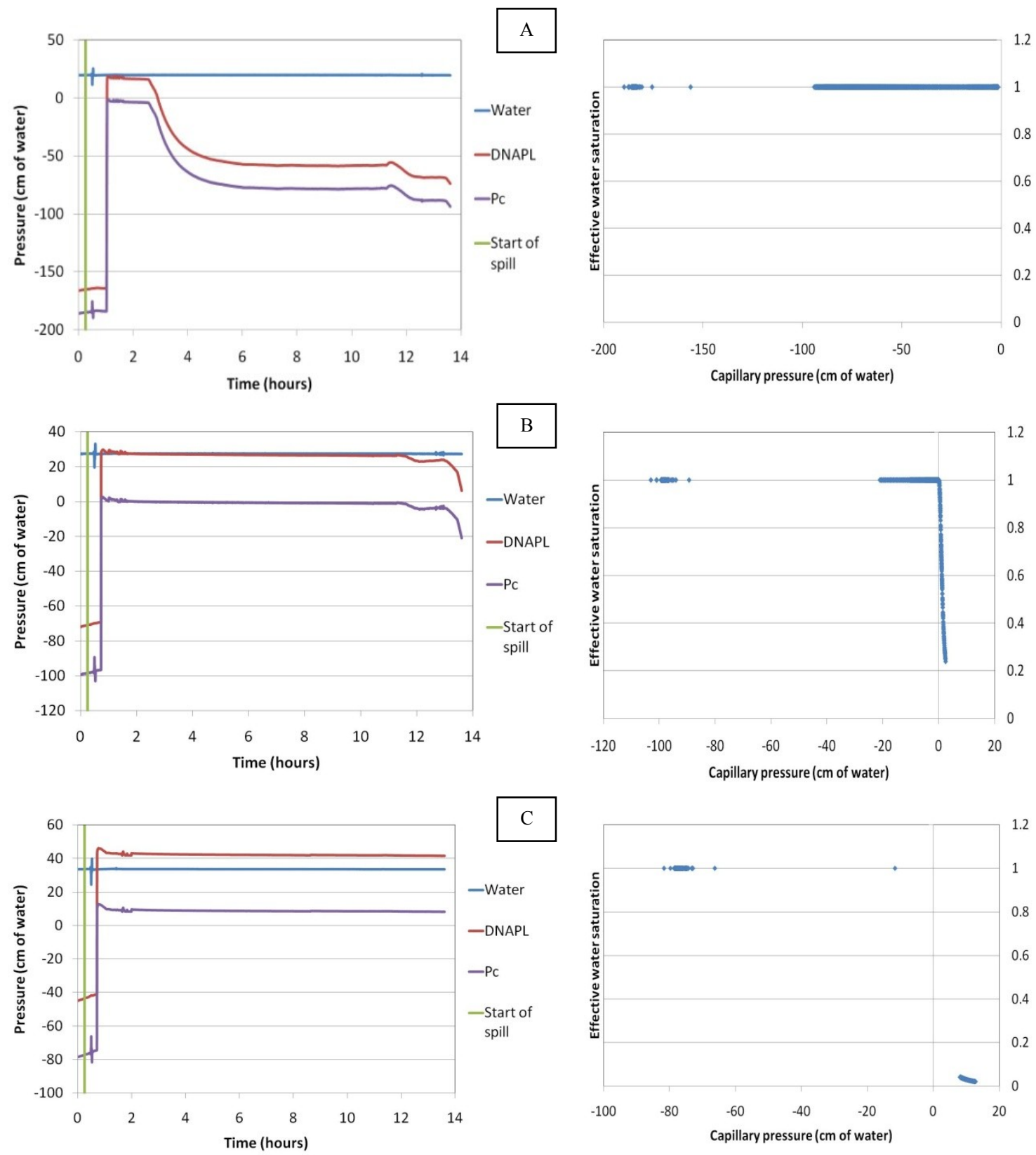

Figure 16: The water, DNAPL and capillary pressure for the spill and the corresponding effective water saturation within the row. A: Row 2, B: Row 3 and C: Row 5, as per Figure 14.

The waterflood did result in the removal of some of the mobile phase DNAPL pooled at the bottom of the box (Figure 17). The amount recovered varied between trials but was between 20 and $9 \mathrm{~mL}$. The photograph indicates that the DNAPL was likely 
removed from the areas where it pooled higher at the sides and middle of the box; there doesn't appear to be any significant removal from the residual DNAPL. This is not unexpected as even at the solubility limit of TCE, $1100 \mathrm{mg} / \mathrm{L}$, only $11 \mathrm{~g}$ would dissolve in the aqueous phase. Resulting in the removal of $7.6 \mathrm{~mL}$, of the $75 \mathrm{~mL}$, of TCE over the entire course of the waterflood. The pressure transducer results (Figure 18), remained relatively constant for row 2 and 5 throughout the entire waterflood. In contrast, although the water pressure was relatively constant the DNAPL pressure in row 3 varied widely throughout the waterflood.
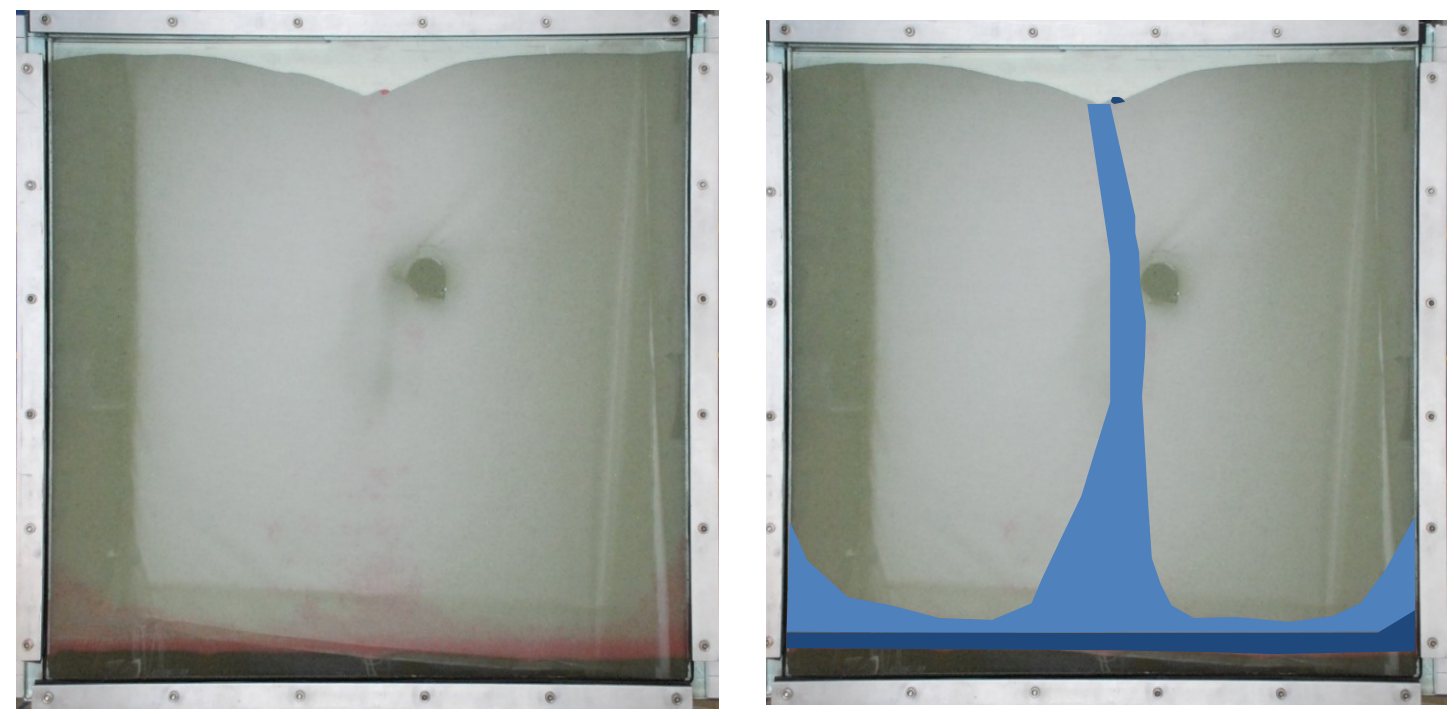

Figure 17: Position of the laboratory grade dyed TCE after a waterflood. In the right hand photograph is a hand drawn interpretation of the DNAPL distribution. The dark blue areas are where DNAPL saturation is high and lighter blue areas indicates areas at which the DNAPL saturation is close to the residual value. 

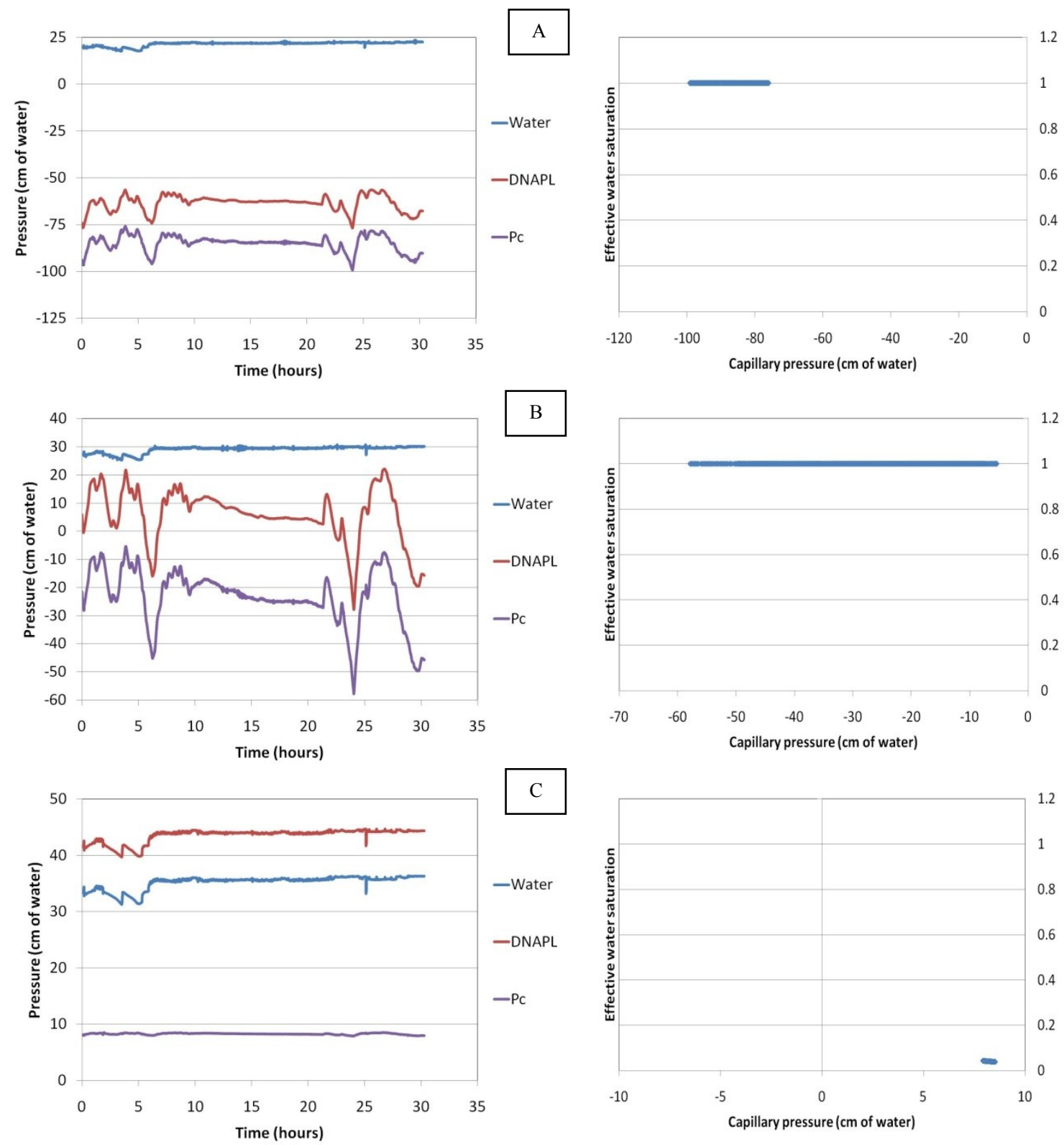

Figure 18: The water, DNAPL and capillary pressure for the waterflood and the corresponding effective water saturation within the row. A: Row 2; B: Row 3 and C: Row 5.

The surfactant flush also resulted in the removal of some the mobile phase DNAPL from certain trials, with a maximum removal of $11 \mathrm{~mL}$, while others had no additional pure phase DNAPL removal. Figure 19 shows the majority of the removal did occur from the pooled DNAPL at the base of the box, although some of the residual DNAPL 
has been removed as well. Pressure transducer results also imply the removal of the residual DNAPL from the middle of the box (Figure 20). Row 5 showed constant pressures until 15 hours after the start of the surfactant flush when the DNAPL pressure decreased and then varied widely for the remainder of the flush. Row 2 showed a slight decrease in DNAPL pressure. Row 3 actually illustrated a large jump in DNAPL pressure after the start of the surfactant flush which quickly decreased and continued that trend for the majority of the rest of the flush. The surfactant flush also resulted in the formation of white foam in the pooled DNAPL (Figure 21).
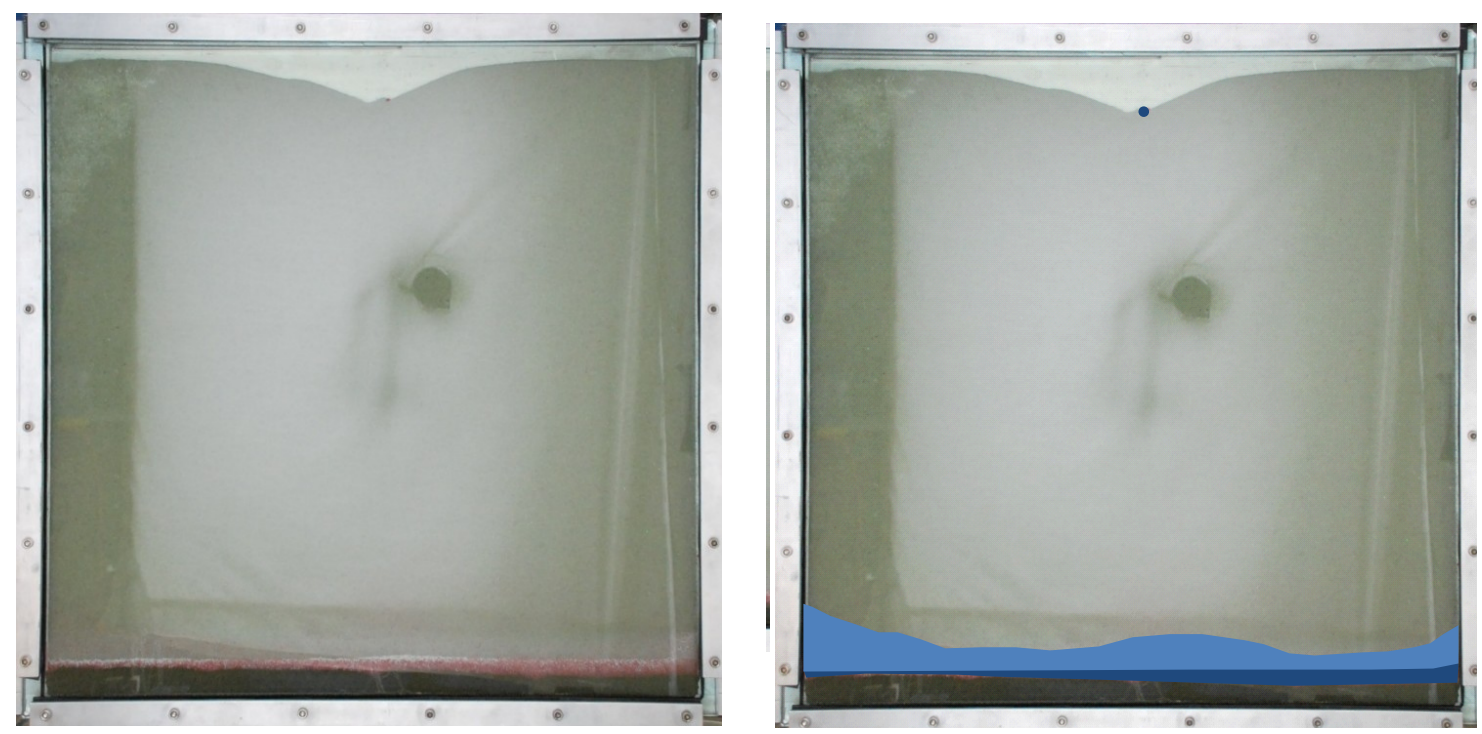

Figure 19: Position of the laboratory grade dyed TCE after a surfactant flush. In the right hand photograph is a hand drawn interpretation of the DNAPL distribution. The dark blue areas are where DNAPL saturation is high and lighter blue areas indicates areas at which the DNAPL saturation is close to the residual value. 

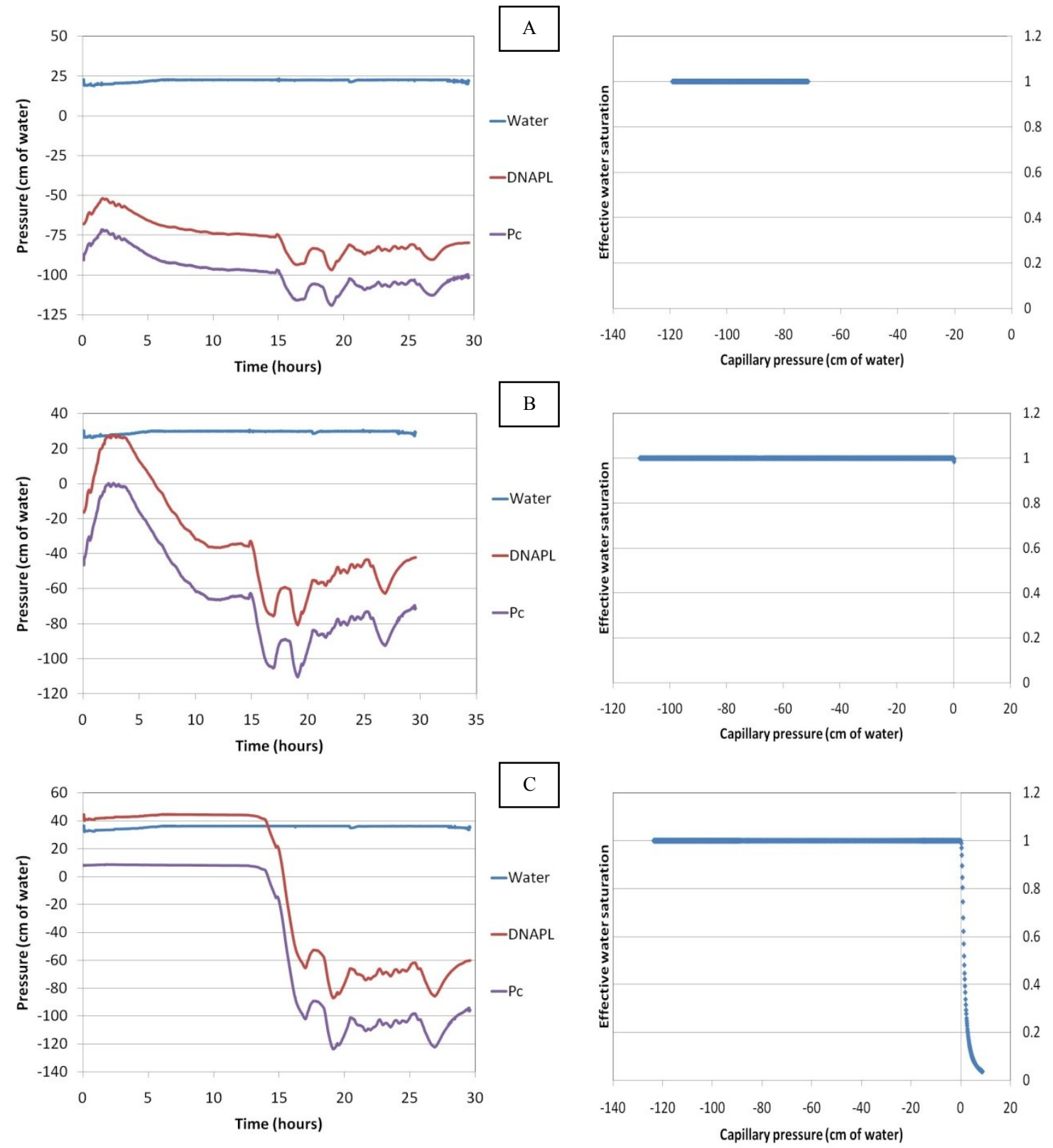

Figure 20: The water, DNAPL and capillary pressure for the surfactant flush and the corresponding effective water saturation within the row. A: Row 2; B: Row 3 and C: Row 5. 


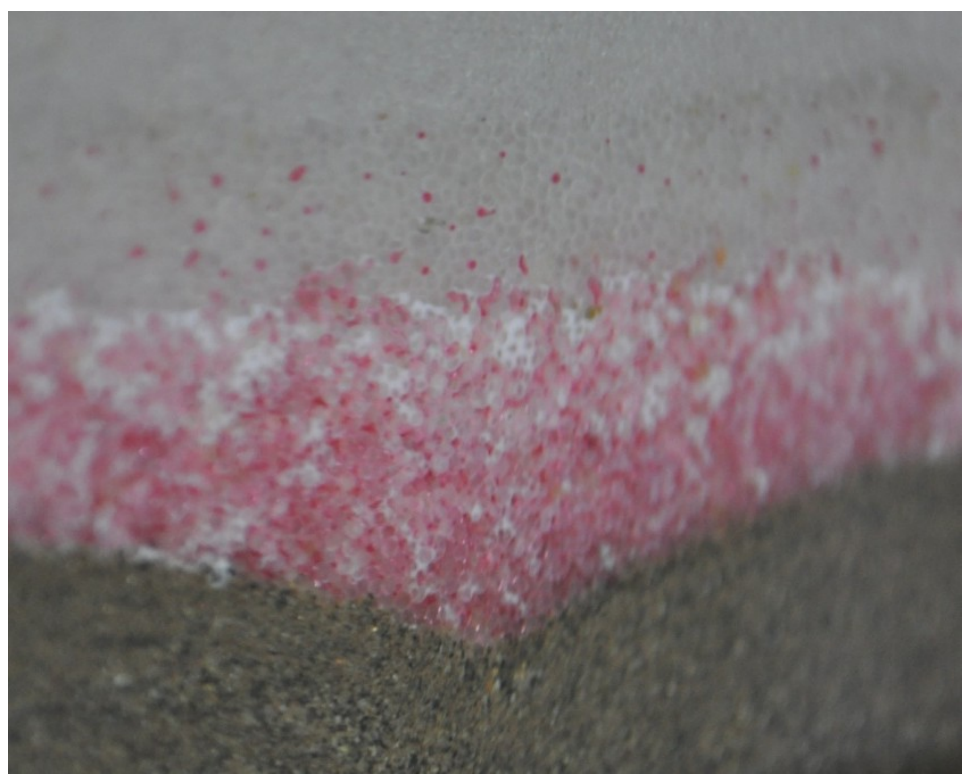

Figure 21: White foam which formed within the pooled DNAPL at the bottom of the box.

\subsection{Hydrophilic sand with fine grained lenses}

Figure 22 was taken prior to the spill, the lighter sand is the fine grained medium. The migration of the DNAPL through the box was very dependent on the shape of the fine grained lenses. Like the homogenous sand the DNAPL migrated downward in a fairly thin line until it came into contact with the top most lens, at which point it began to pool. As the pool height increased the DNAPL also began to spread laterally until it reached the edges of the lens; in one trial the lens was angled in such a way that the DNAPL only cascaded over one side of the lens. After reaching the edge of the lens the DNAPL continued its downward migration to the second lens, where once again it began to pool and migrate laterally to the closest edge of the lens. Once again the DNAPL continued its downward migration and depending on the length of the lenses, it would 
either hit, pool and migrate laterally to the edge of the lens or migrate directly to the bottom of the box where it would pool and then migrate laterally (Figure 23).

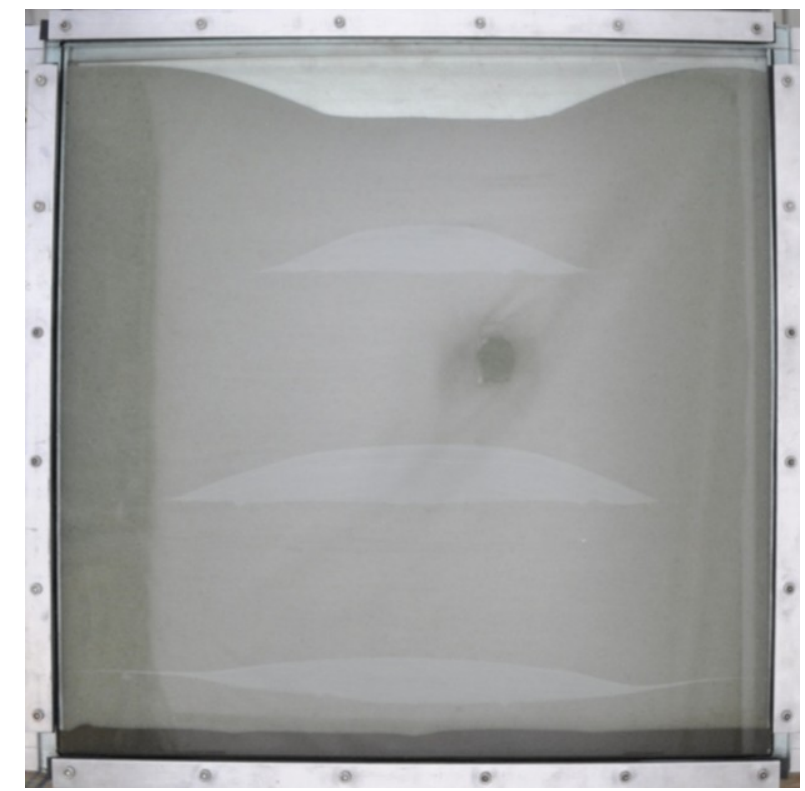

Figure 22: Image of the box prior to the spill in homogeneous hydrophilic sand with fine grained lenses, the lighter coloured areas are the fine grained lenses.
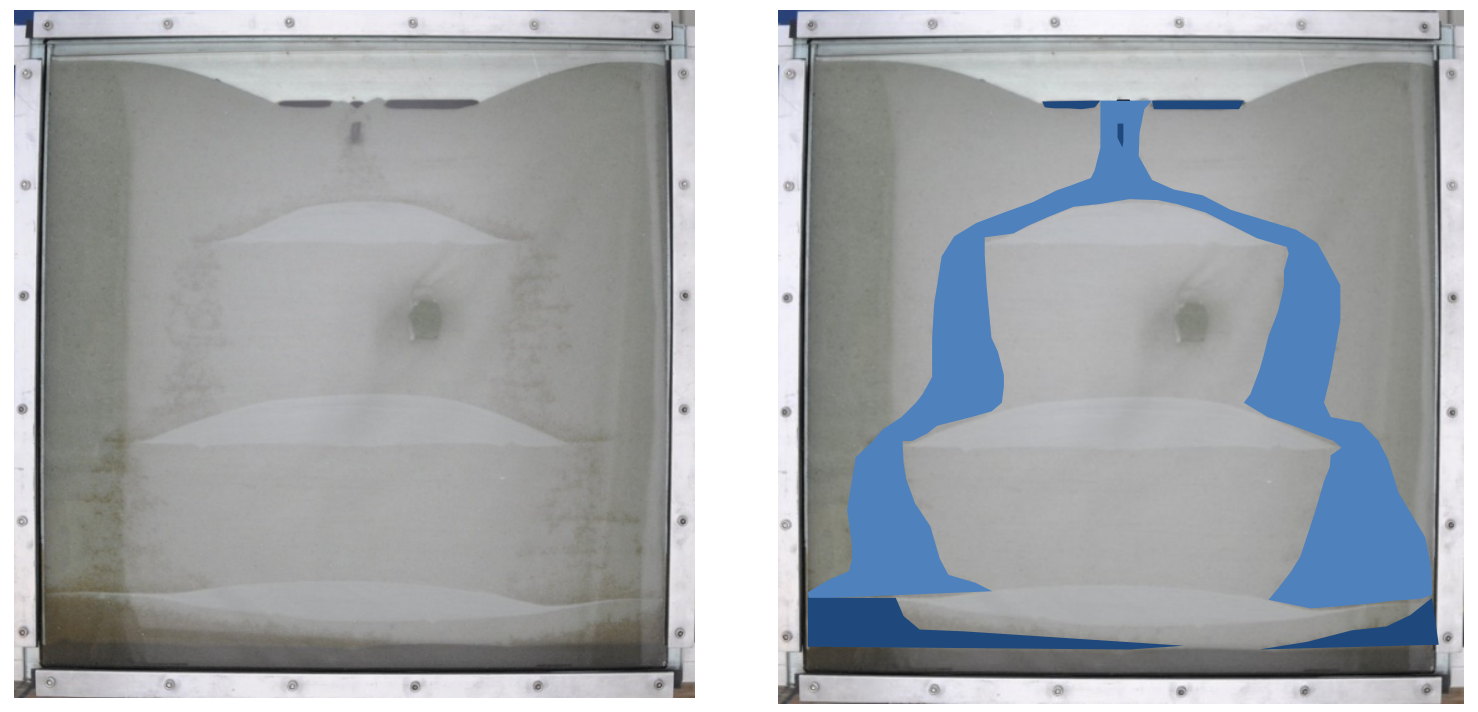

Figure 23: Position of the field DNAPL after a spill in hydrophilic media with fine grained lenses (12 hrs). In the right hand photograph is a hand drawn interpretation of the DNAPL distribution. The dark blue areas are where DNAPL saturation is high and lighter blue areas indicates areas at which the DNAPL saturation is close to the residual value. 
The waterflood did not result in any significant changes in the DNAPL distribution in the box (Figure 24). Although in half the trials some free phase DNAPL was recovered it was a minimal amount, less than $5 \mathrm{~mL}$; this was likely a result of the DNAPL pooling higher closer to the outlet and likely entering the manifold prior to the start of the waterflood.
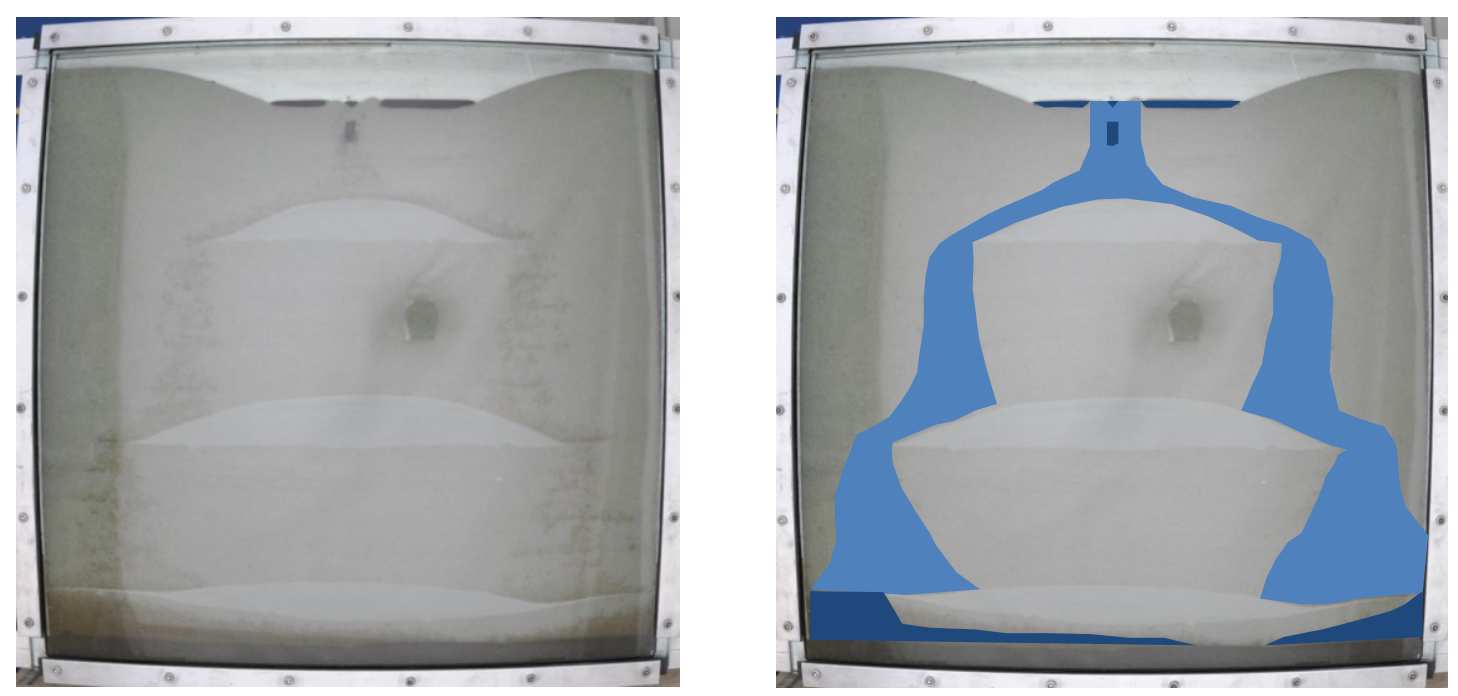

Figure 24: Position of the field DNAPL after a waterflood in hydrophilic media with fine grained lenses. In the right hand photograph is a hand drawn interpretation of the DNAPL distribution. The dark blue areas are where DNAPL saturation is high and lighter blue areas indicates areas at which the DNAPL saturation is close to the residual value.

The surfactant flood did result in the removal of some of the DNAPL pooled at the base of the box and some of the residual within the box; although some DNAPL was left on the fine grained lenses (Figure 25). Figure 26 illustrates the common transducer behaviour during the experiments, some trials did show more variations but there were no trials in which the DNAPL pressure reached a level resulting in positive capillary pressure. 

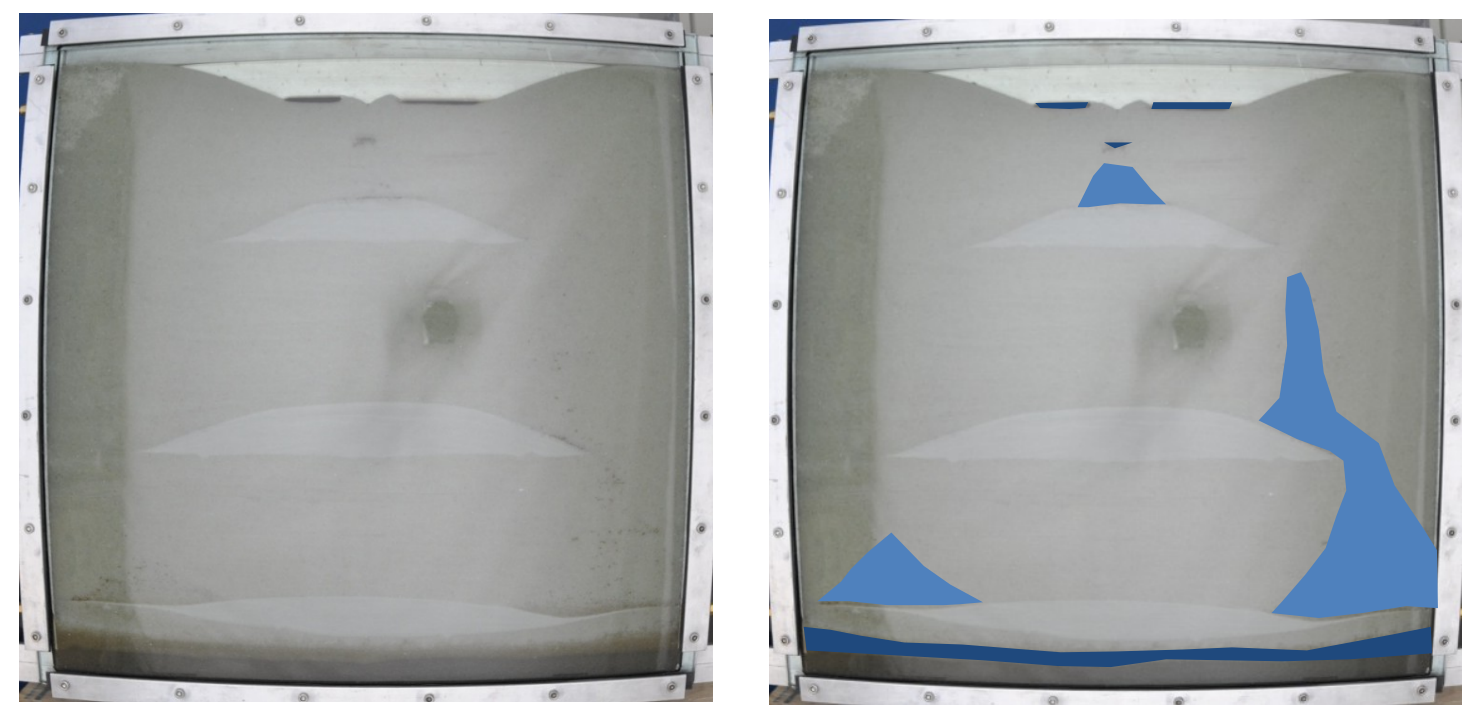

Figure 25: Position of the field DNAPL after a surfactant flush in hydrophilic media with fine grained lenses. In the right hand photograph is a hand drawn interpretation of the DNAPL distribution. The dark blue areas are where DNAPL saturation is high and lighter blue areas indicates areas at which the DNAPL saturation is close to the residual value. 


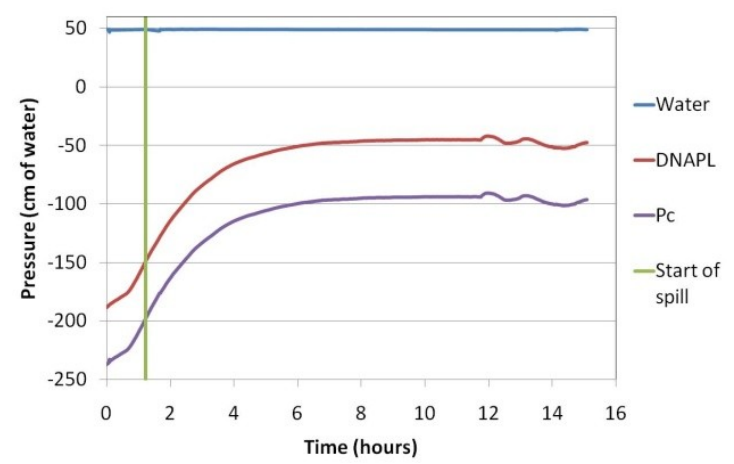

Spill

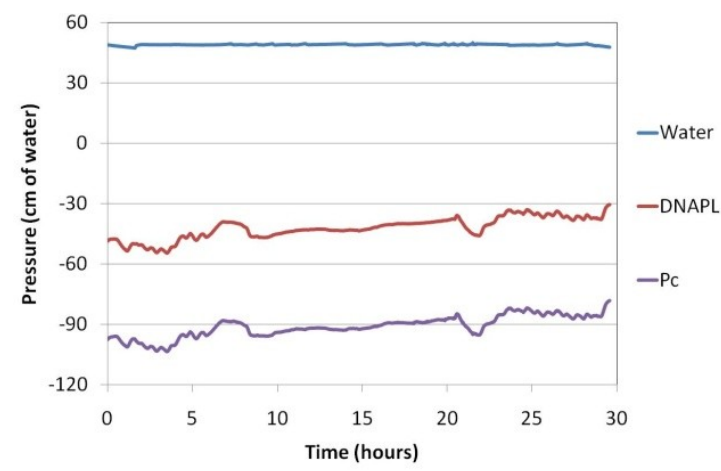

Waterflood

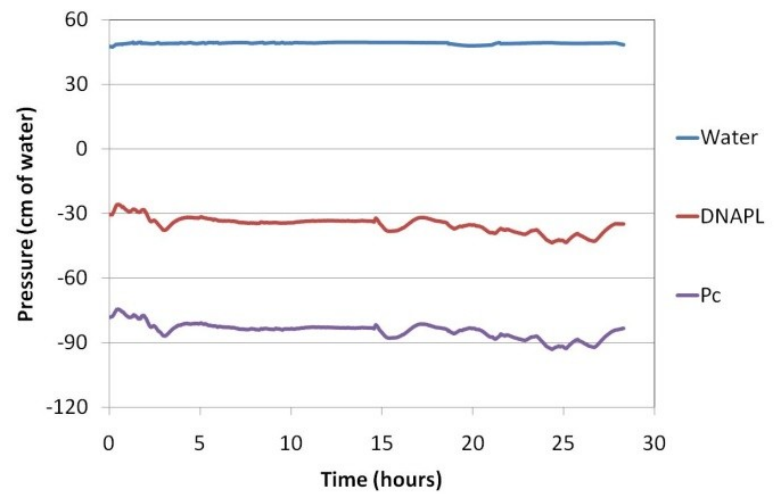

Surfactant flush

Figure 26: The water, DNAPL and capillary pressure for Row 7 (located $9.5 \mathrm{~cm}$ from the bottom of the box) during the entire experiment. The pressure remained fairly constant throughout which was common for all rows during all trials. 


\subsection{Hydrophilic sand with hydrophobic lenses}

Figure 27 and Figure 28A contain images of the experimental box just prior to the commencement of the spill; the lenses of hydrophobic material were slightly different in colour due to the OTS treatment.

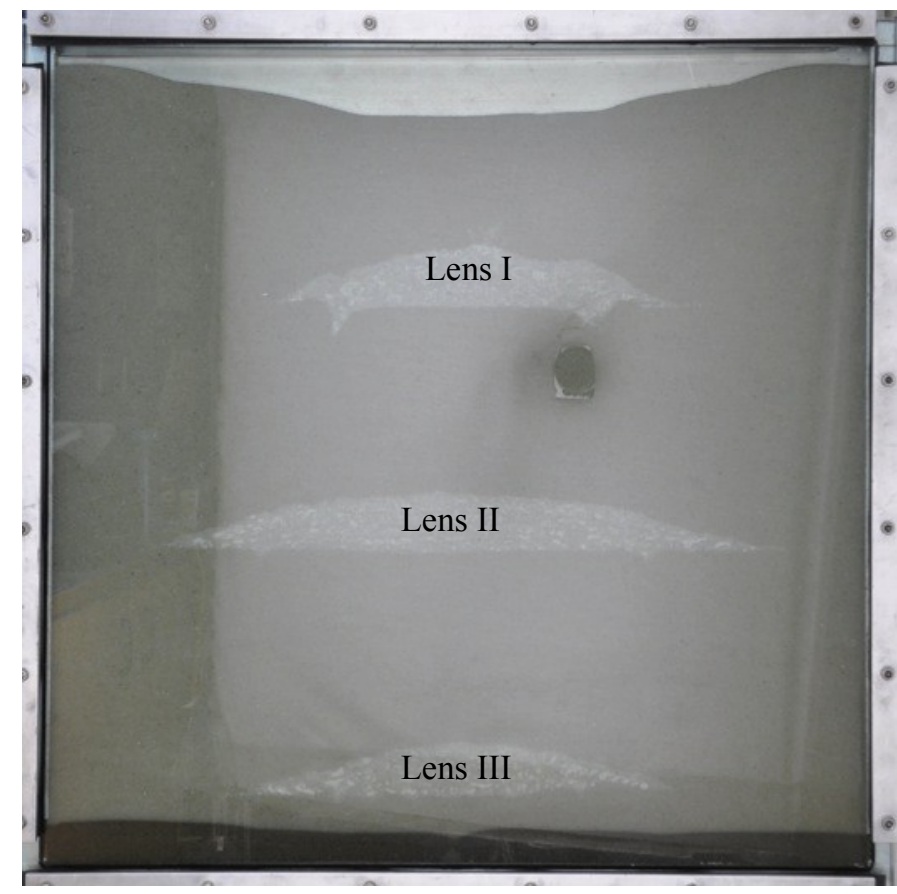

Figure 27: Image of the sand box prior to the spill of field DNAPL in hydrophilic sand containing 3 hydrophobic lenses. Note the lighter mateial is the hydrophobic sand and the black circle below lens I is the reflection of the camera lens. 


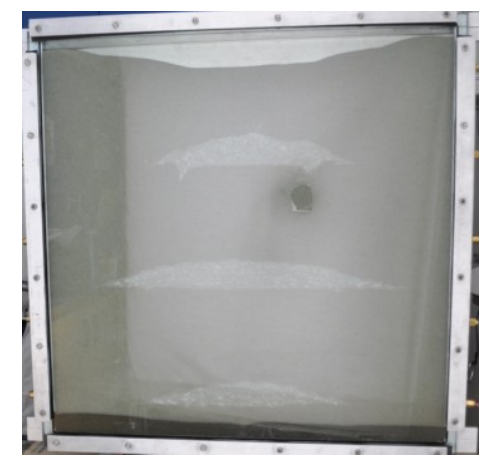

A

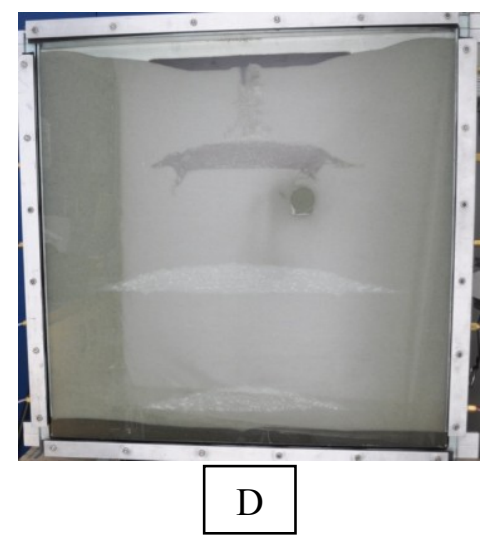

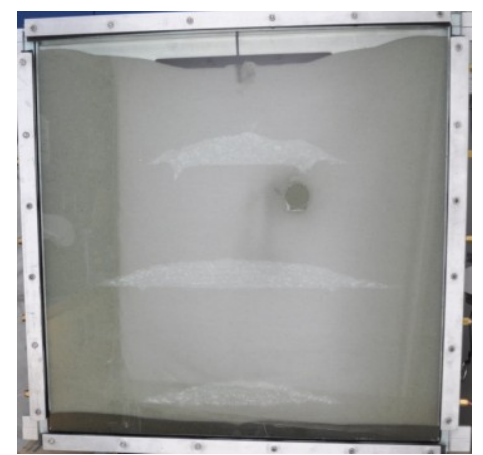

$\mathrm{B}$

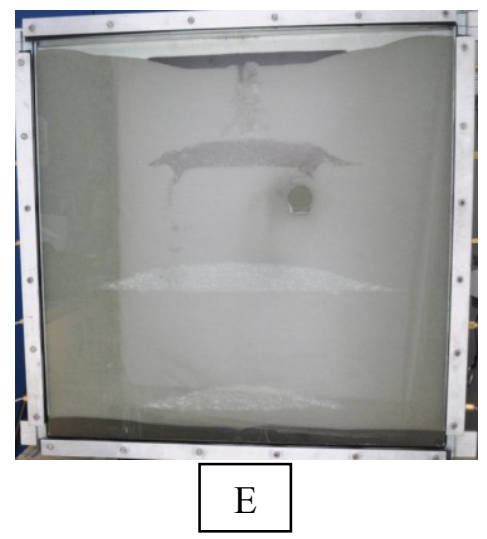

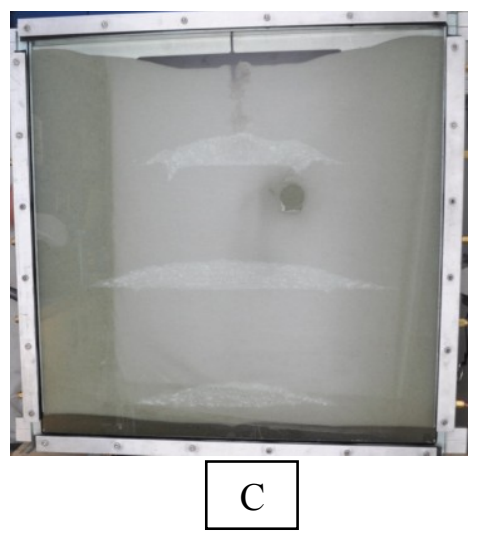

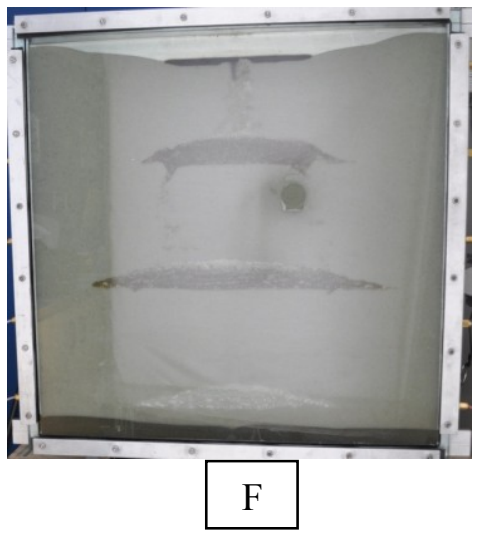

Figure 28: Time lapse photography of a spill with hydrophobic lenses. A: Box prior to the spill. B: DNAPL overcomes the entry pressure and begins to invade the underlying hydrophilic soil, photo taken 50 minutes after the spill began. C: DNAPL begins to invade the top hydrophobic lens, photo taken 80 minutes after the spill began. D: Top hydrophobic lens is saturated and begins to drain into the underlying hydrophilic media; photo taken approximately 4 hours after the spill began. E: DNAPL begins to invade the second hydrophobic lens; photo taken 4 hours and 20 minutes after the spill began. F: Photo taken at the end of the spill period, 13.5 hours after the spill began.

After the entry pressure was achieved, the DNAPL flowed downward towards lens I (Figure 28B). In this particular experiment, the DNAPL came into contact with the lens only after the entire $75 \mathrm{~mL}$ of DNAPL had been spilled. Once in contact with the lens, the DNAPL was quickly drawn into the lens and the DNAPL and capillary pressures increased sharply (Figures $28 \mathrm{C}$ and 29A). It is important to note the capillary pressuresaturation curve for the NAPL-wet soil is shifted below the zero-value for capillary 
pressure; i.e. the soil goes from water saturated to NAPL saturated as the capillary pressure approaches zero capillary pressure. In contrast to the capillary pressuresaturation for the water-wet soil (see Figure 15 - plot C for example) which is $100 \%$ water saturated until the capillary pressures reach positive values and exceed the NAPL entry pressure after which point the NAPL displaces the water. After approximately 4 hours, the DNAPL at the base of lens I entered the underlying hydrophilic sand indicating that the DNAPL pressure exceeded the NAPL entry pressure of the hydrophilic sand below (Figure 28D). After overcoming the underlying hydrophilic soil entry pressure it took a further 20 minutes for the DNAPL to enter the second lens (Figures 28E and 29B). The pressure within lens II was never high enough to overcome the entry pressure of the underlying hydrophilic media and as such halted any further downward migration of the DNAPL as can be seen in Figures 28F, 29C and 30. 

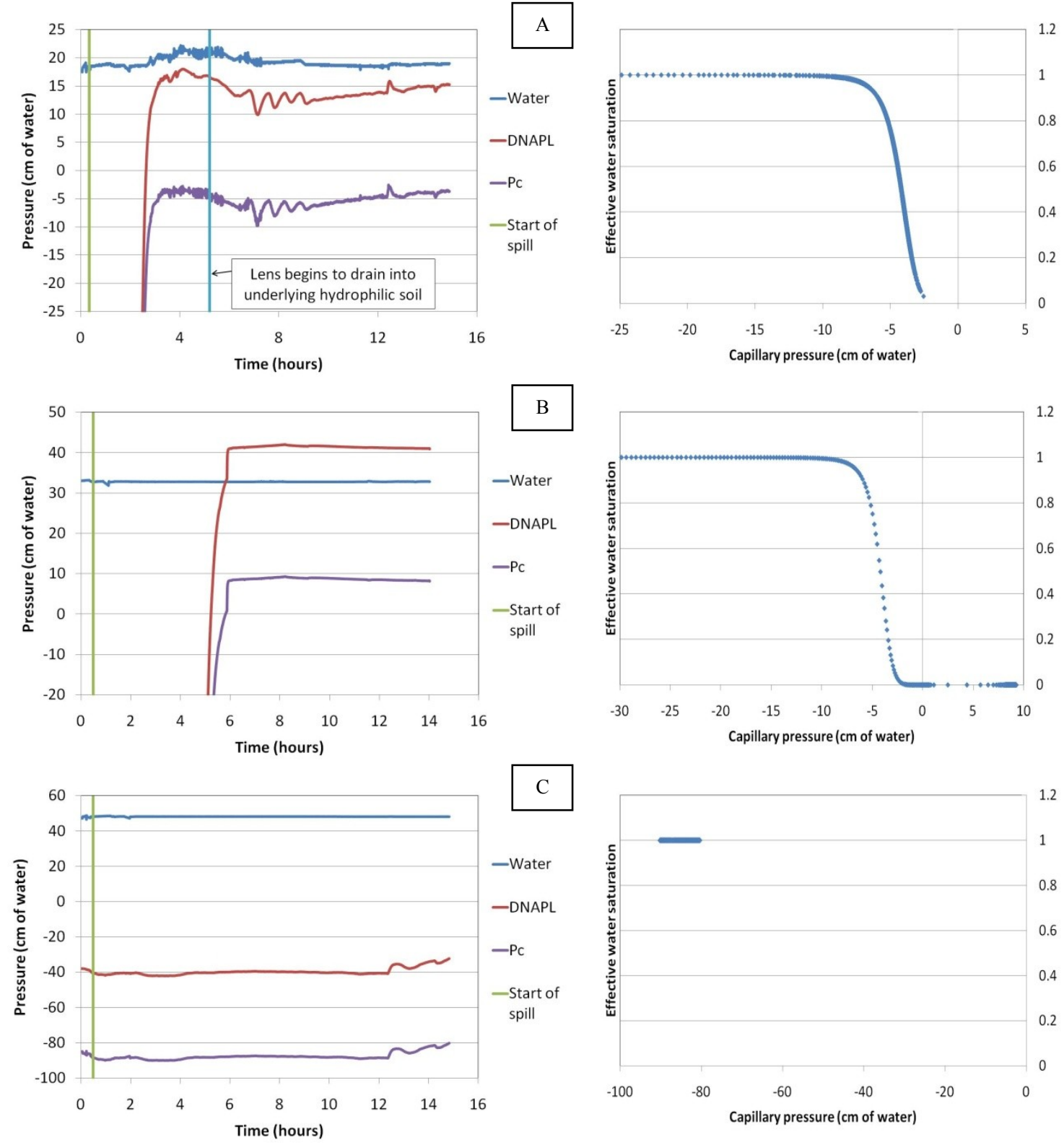

Figure 29: The water, DNAPL and capillary pressure for the spill and the corresponding effective water saturation within the lenses. A: Lens I; B: Lens II and C: Lens III. Note for lenses I and II the pressure axes in the plots have been truncated to allow better visualization of the data; as the strongly negative pressures of the DNAPL prior to the arrival of the DNAPL do not reflect actual DNAPL pressures. 

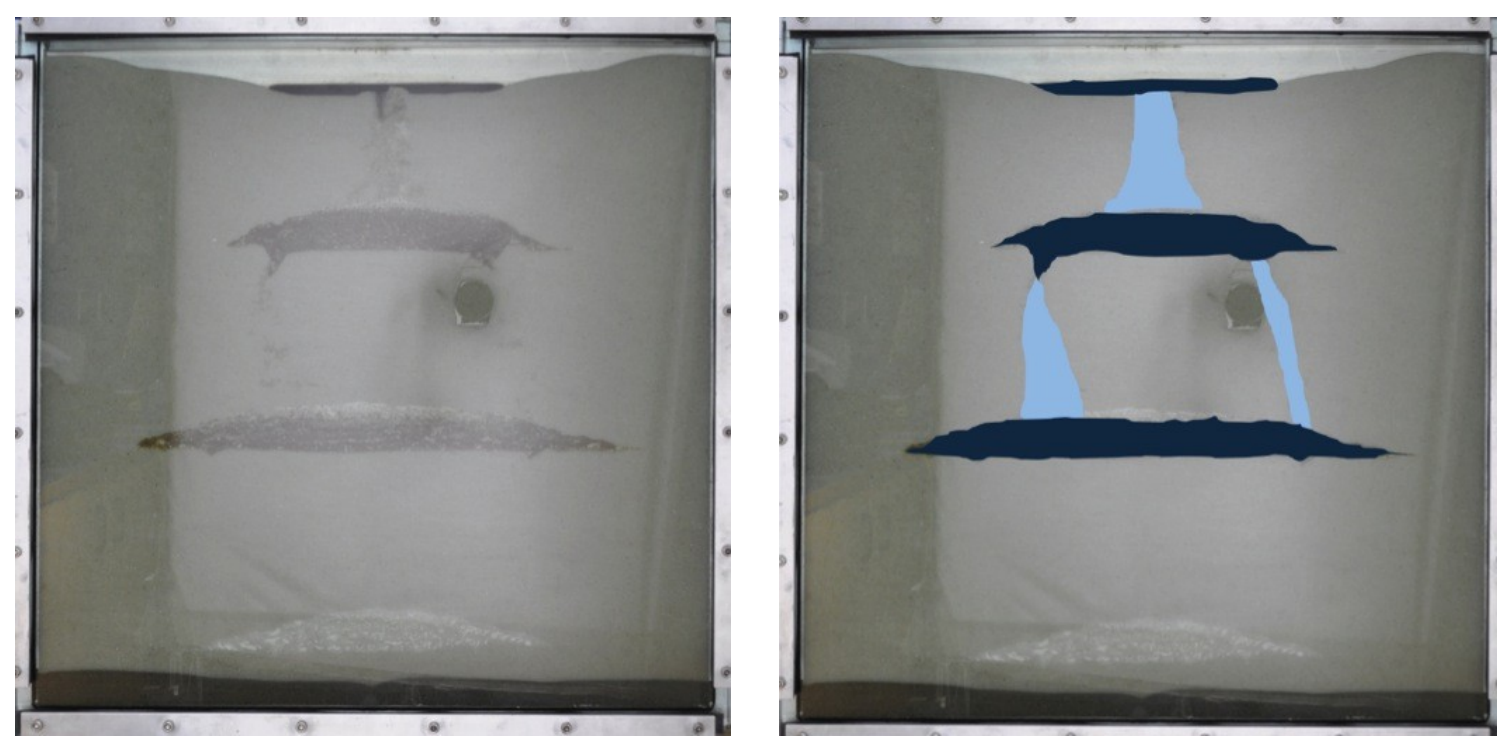

Figure 30: Position of the field DNAPL after a spill (12 hrs). In the right hand photograph is a hand drawn interpretation of the DNAPL distribution. The dark blue areas are where DNAPL saturation is high and the lighter blue indicates areas at which the DNAPL saturation is close to the residual value.

Figure 31 presents the static pressure distribution within lens I, at the end of the spill period, showing that while the capillary pressure measured by the transducers at the centre of the lens indicate a water pressure greater than the NAPL pressure, at the lowest point of lens I the NAPL pressures could exceed the water pressures. The capillary pressures may not exceed the $2 \mathrm{~cm}$ NAPL entry pressure referenced earlier; however, the entry pressure may be slightly lower at this location as the hydrophilic and hydrophobic sands may have mixed as the plastic dividers were removed during sand placement creating a mixed wettability zone or the sand packing may be looser creating a lower entry pressure. In either case, the entry pressure was exceeded and the mobile phase DNAPL continued its downward migration. 


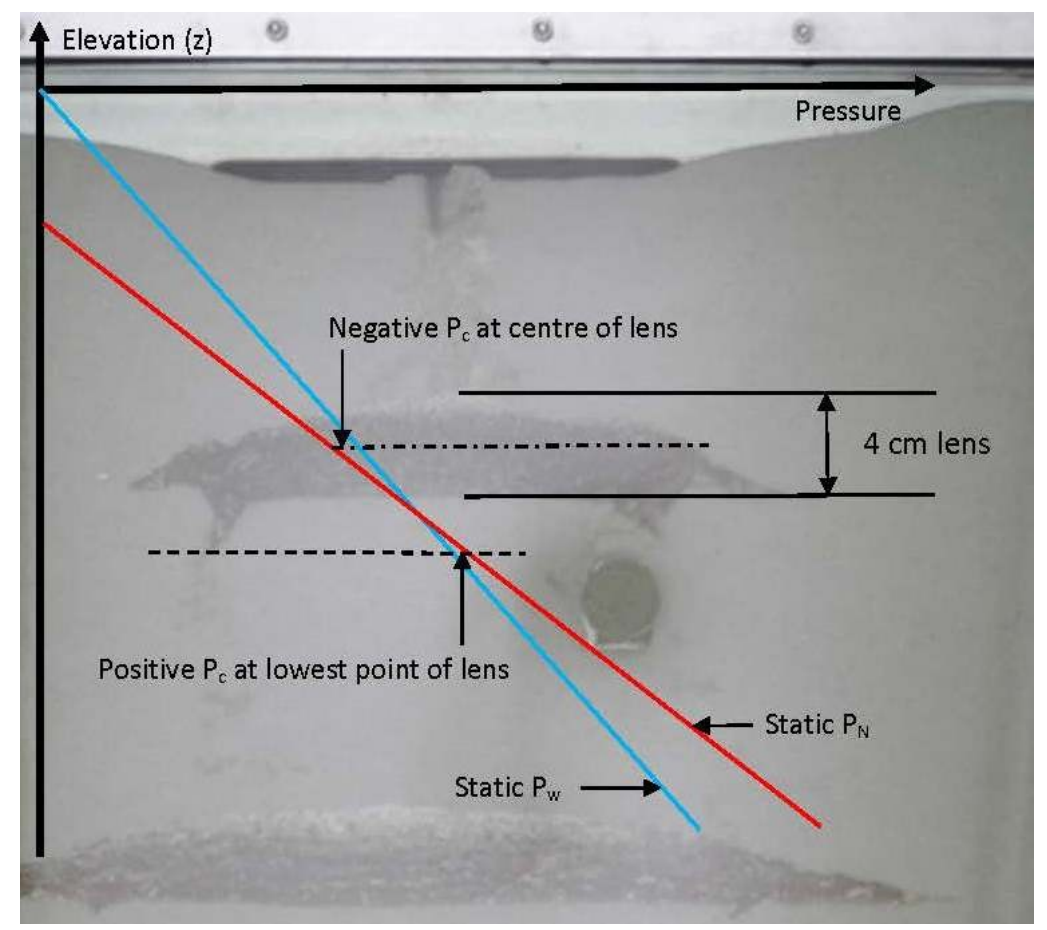

Figure 31: Schematic of the estimated pressure distribution in lens I after the spill.

As with lens I, once the DNAPL came in contact with the lens II, the DNAPL was drawn into the lens and the capillary pressure responded accordingly (Figure 29B). Unlike lens I, the DNAPL did not migrate into the underlying hydrophilic soil suggesting that the DNAPL in lens II could not overcome the entry pressure of the hydrophilic material below. However, the transducers at the centre of lens II indicate that the NAPL pressure is approximately $8 \mathrm{~cm}$ greater than the water pressure and based on static pressures, this pressure difference would only increase with depth within the lens. Similar capillary pressures were recorded in all four spills experiments and ranged from 6-8 cm. It is believed the DNAPL is trapped within the lens. While the DNAPL exceeds the NAPL entry pressure of the hydrophilic sand below, the water pressure within the lens does not exceed the water entry pressure of the hydrophobic sand allowing the 
DNAPL to drain; hence, the DNAPL appears to be trapped. This is discussed further in the context of the surfactant flush. Pressure transducer data also indicate that the DNAPL never reached lens III located at the bottom of the box (Figure 29C).

A photograph taken at the end of the spill and after the DNAPL has redistributed itself (Figure 30) corroborates the pressure readings recorded during the spill, with lenses I and II showing the colour change indicating that they had been invaded by the DNAPL. The image on the right in Figure 30 illustrates the approximate DNAPL distribution. Approximately $20 \mathrm{~mL}$ of DNAPL remained at the spill location. Given a porosity of 0.36 and an assumed residual water saturation of $20 \%$ in the DNAPL-saturated portions of the hydrophobic lenses, it was estimated that approximately $22 \mathrm{~mL}$ and $30 \mathrm{~mL}$ of DNAPL is held in lens I and II, respectively, leaving only $3 \mathrm{~mL}$ of DNAPL trapped in the hydrophilic soil between the spill area and lens I and between lenses I and II. Over the course of the spill period, very few of the other DNAPL transducers outside the hydrophobic lenses recorded any significant changes in pressure. In two spills, the topmost transducer in the hydrophilic medium recorded an increase in DNAPL pressure during the spill which either subsequently decreased to a reading below zero prior to the commencement of the waterflood or remained at a steady level throughout the three experimental phases. The only other location which displayed a significant increase in DNAPL pressure during the spill was the DNAPL transducer located between lenses I and II. This particular transducer recorded a spike in pressure as the DNAPL front moved downward and after this spike, only recorded negative DNAPL pressure.

After the twelve hour spill period, the waterflood was initiated. During the waterflood, the DNAPL pressures were largely unchanged in all experiments. In lens I 
(Figure 32A), the DNAPL and water pressures did show some minor variations. These variations do indicate changes to the level of water saturation in the lens based on the pressure data and the $\mathrm{P}_{c}-\mathrm{S}$ relationship; however, given the measurement accuracy of the two transducers and the relatively flat $\mathrm{P}_{\mathrm{c}}-\mathrm{S}$ relationship for this uniform sand, it is believed that the water and DNAPL saturations did not vary significantly. The other experiments indicated similar results for the transducers within lens I and the capillary pressures for all experiments varied between -5 and $+5 \mathrm{~cm}$ of equivalent water pressure. In contrast, lens II (Figure 32B), showed very little variation throughout the waterflood and in every case, once saturated by the DNAPL, the capillary pressures remained positive corresponding to an effective water saturation at the centre of lens II of $0 \%$. During the waterflood, the transducers located at the bottom of the box did not indicate the arrival of any DNAPL (Figure 32C). In only one case did the waterflood affect the DNAPL pressure and result in an effective water saturation in lens III that was less than $100 \%$. Figure 33 is a photograph taken at the end of a waterflood and there is little difference between this image and the images after the spill presented in Figure 30. The pooled DNAPL remained at the top of the sand and the entrapped DNAPL between lenses remained largely unchanged. 

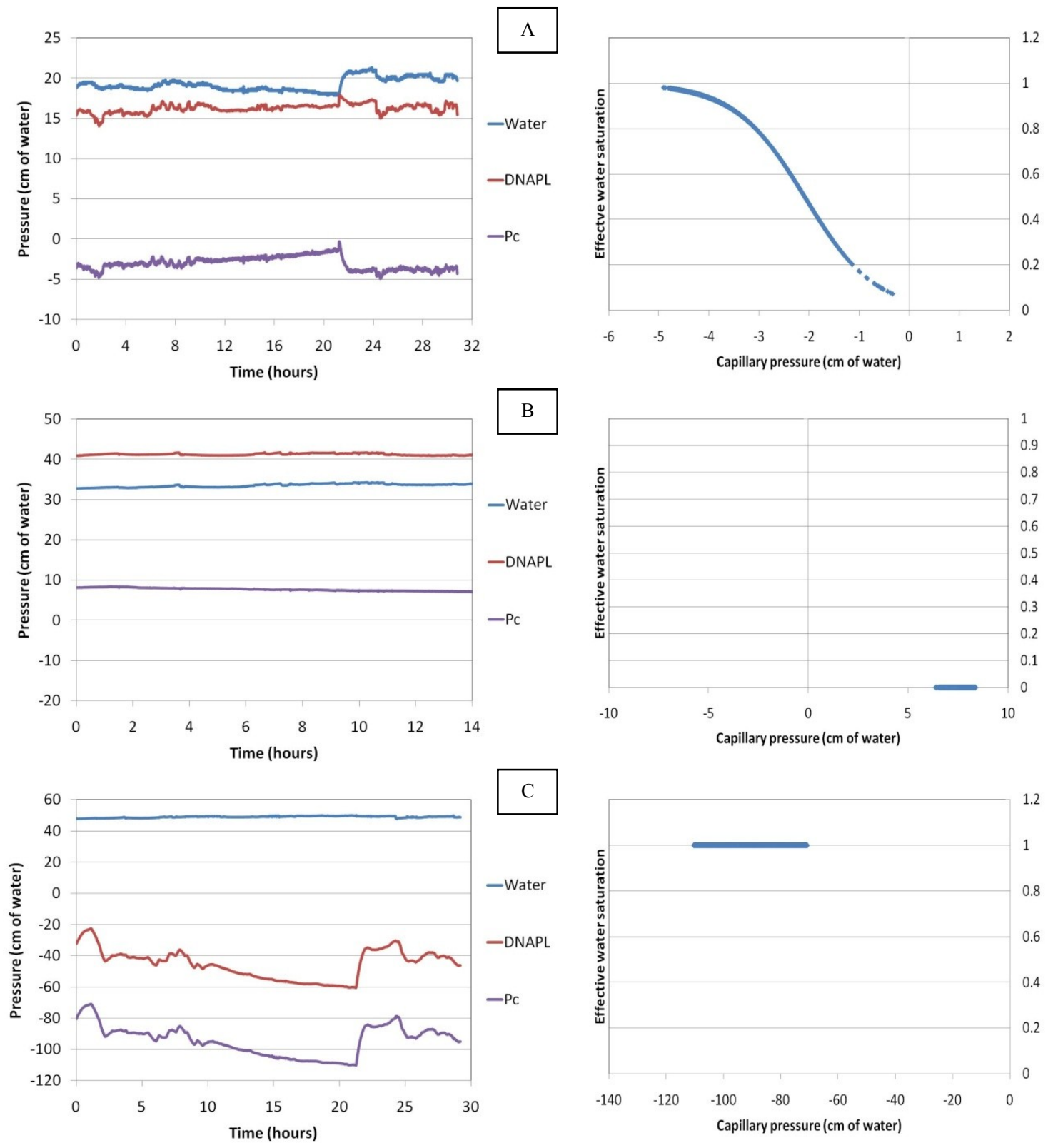

Figure 32: The water, DNAPL and capillary pressure for the waterflood and the corresponding effective water saturation within the lenses. A: Lens I; B: Lens II and C: Lens III. 

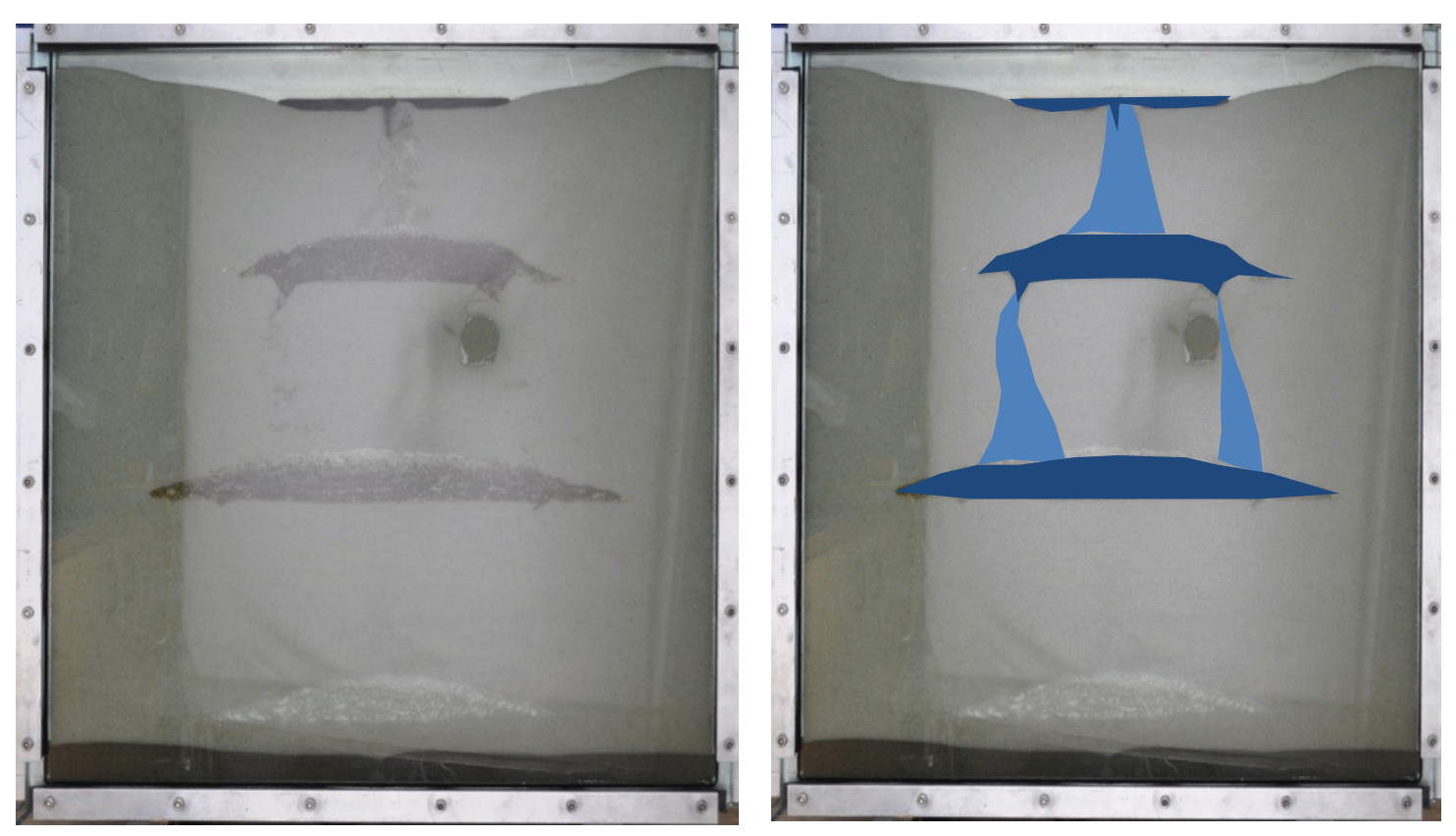

Figure 33: Position of the field DNAPL after the waterflood. In the right hand photograph is a hand drawn interpretation of the DNAPL distribution. The dark blue areas are where DNAPL saturation is high and the lighter blue indicates areas at which the DNAPL saturation is close to the residual value.

The surfactant flush immediately followed the waterflood and resulted in significant changes in the distribution of the DNAPL within the box (Figures 34, 35 and 36). It is clear from Figures 34, 35 and 36 that the surfactant reduced the water-DNAPL interfacial tension causing the DNAPL within the source area (pool) and the lenses to mobilize. Lens I displayed a considerable variation in DNAPL pressure (Figures 34 and 35A), particularly when assessed against the waterflood. It is clear that when the interfacial tension was reduced, the NAPL partially drained from lens I and the NAPL pressure sharply decreased at the centre of the lens. After the decrease in NAPL pressure, the capillary pressures reached $-20 \mathrm{~cm}$ indicating an effective water saturation of $100 \%$ and residual DNAPL saturation. Following this decrease, it is believed the 
NAPL transducers are no longer connected to any mobile DNAPL and the variations in DNAPL pressure do not reflect saturation changes. It is clear from the image of the box taken at the conclusion of the surfactant flush (Figure 34) that the lower portion of lens I appears to remain saturated indicating a capillary barrier still remained limiting complete drainage of the DNAPL.

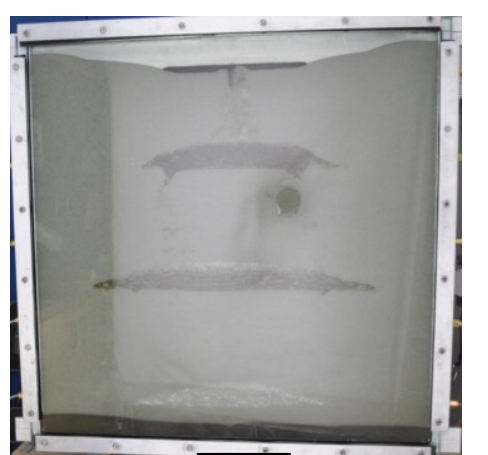

A

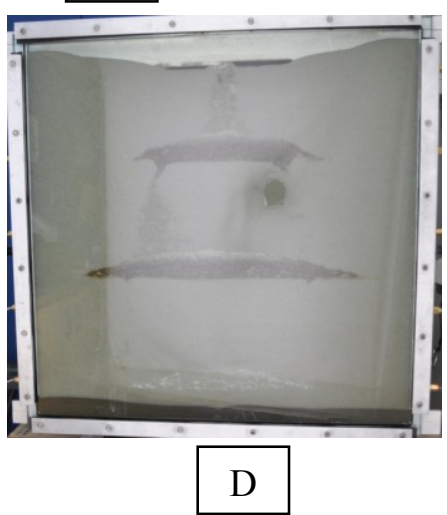

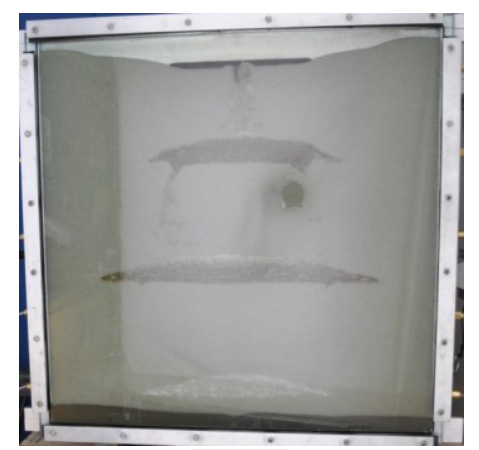

B

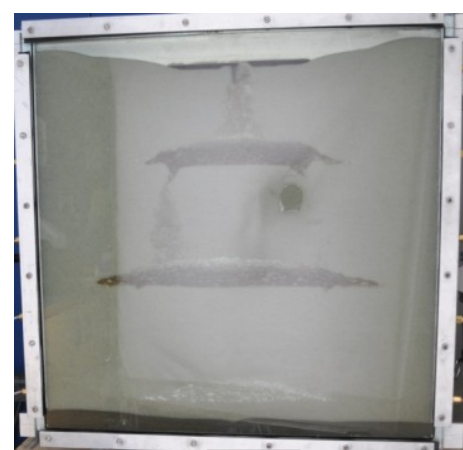

C

Figure 34: Time lapse photography of a surfactant flush with hydrophobic lenses. A: Box at the end of the waterflood and prior to the surfactant flush. B: The saturated top lens begins to drain again into the underlying hydrophilic soil; this photo was taken approximately 3 hours after the previous photo. C: The DNAPL pool at the interface between the water and porous media begins to drain, photo taken 3.5 hours after the previous photo. D: The second lens begins to drain to drain into the underlying hydrophobic media, photo taken 1 hour after the previous photo. E: Photo taken at the end of the surfactant flood, 7 hours after the previous photo. 

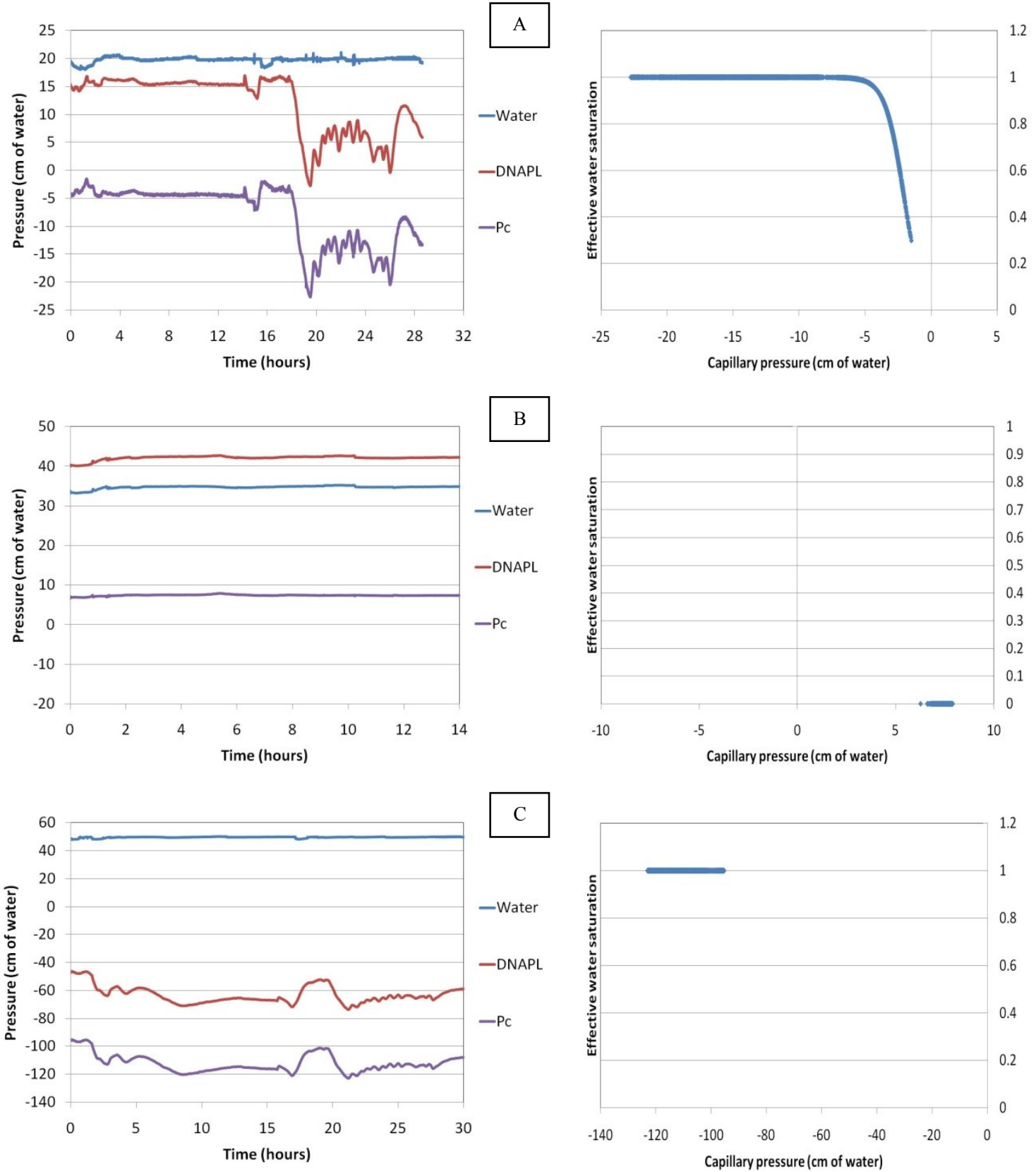

Figure 35: The water, DNAPL and capillary pressure for the surfactant flush and the corresponding effective water saturation within the lenses. A: Lens I; B: Lens II and C: Lens III. 

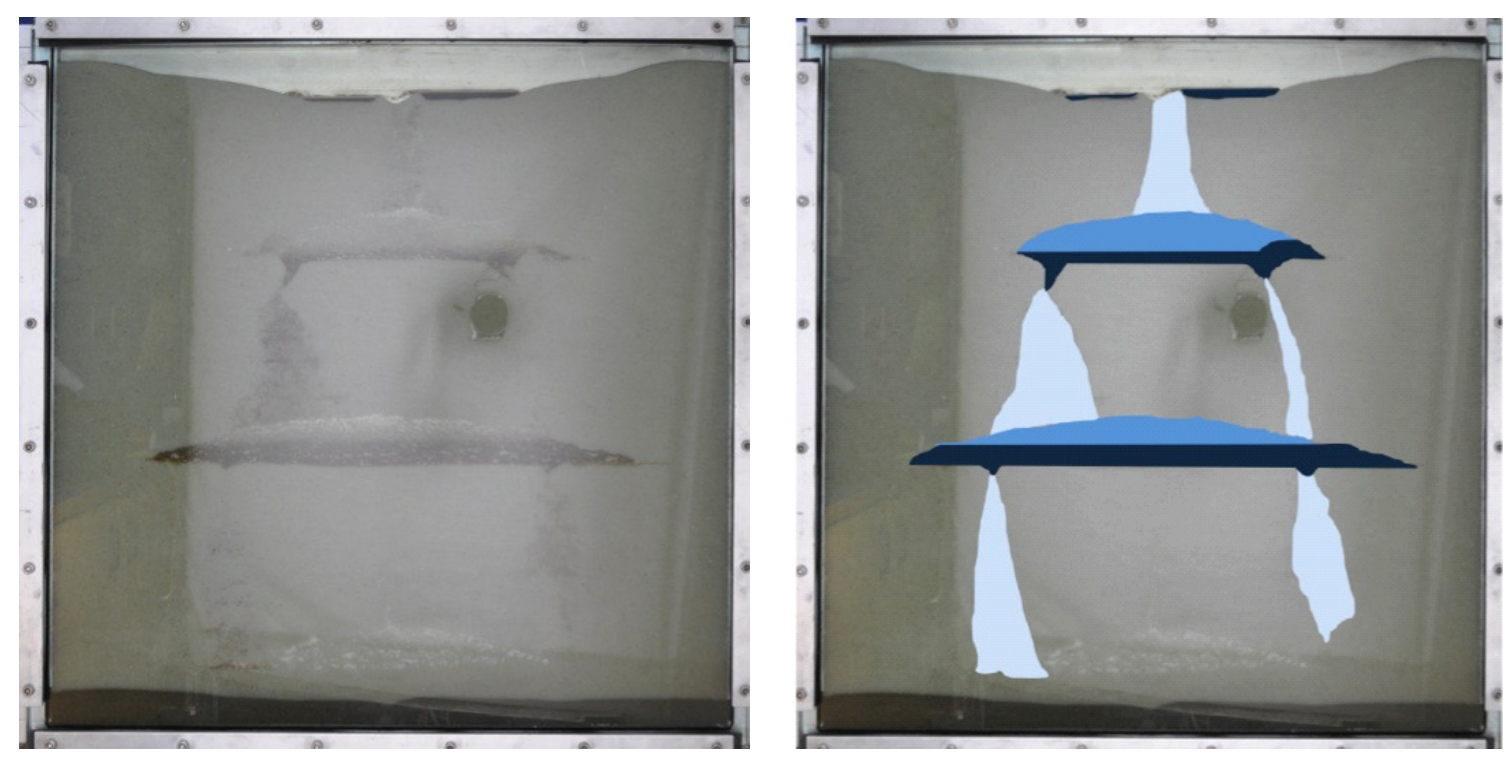

Figure 36: Position of the field DNAPL after a surfactant flush. In the right hand photograph is a hand drawn interpretation of the DNAPL distribution. The dark blue areas are where DNAPL saturation is high, the medium blue designates areas within the hydrophobic lenses from which the DNAPL has drained and the lighter blue indicates areas at which the DNAPL saturation is close to the residual value.

The capillary pressures recorded at the centre of lens II in the box (Figure 35B) indicated that this location remained saturated with DNAPL during the entire surfactant flush, excluding one trial in which the capillary pressures did decrease indicating that the DNAPL did drain from the area in which the transducers were located near the centre of lens II. Figure 36 denotes the distribution of DNAPL after the surfactant flush. Three distinct zones appear in lens II. The top portion of the lens remains water saturated as it appeared after the spill (Figure 30) and after the waterflood (Figure 33). Similarly the lower half of the lens remained NAPL saturated. A zone exists between these two zones and above the pressure transducer locations, where the DNAPL saturations appear to decrease to a residual DNAPL saturation. In all but one experiment, the transducers at the centre of lens III (Figure 35C) indicated that the DNAPL did not reach this location. 
However, the DNAPL distribution after the surfactant flush (Figures 34 and 36) indicates that some DNAPL migrated below lens II and reached lens III.

Earlier in the discussion of the spill results, it was noted that the capillary pressures at the centre of lens II remained at approximately $8 \mathrm{~cm}$ after the DNAPL reached lens II and during the waterflood and it was hypothesized that the DNAPL was trapped within lens II even though the DNAPL pressure exceeded the entry pressure of the hydrophilic sand below. It is important to note that during the surfactant flush, the capillary pressures at the centre of lens II remained at approximately $8 \mathrm{~cm}$ even though a portion of the DNAPL drained from lens II. Similar results were seen for three of the four spills; in the one experiment in which the DNAPL pressures decreased, it was assumed that the transducers were located in the middle zone in which the DNAPL drained. One might have expected the capillary pressure within the lens to decrease given the reduction in interfacial tension at the water-NAPL interface above and below the transducer locations causing the DNAPL to partially drain. However, it appears that the DNAPL remains trapped and at a capillary pressure of $8 \mathrm{~cm}$, consistent with the results during the spill and waterflood. The surfactant appears to have reduced the interfacial tension causing the thickness of the NAPL saturated zone to decrease. That thickness may now reflect the capillary pressure difference between the reduced NAPL-entry pressure at the lowermost hydrophobic-hydrophilic soil interface and the reduced water-entry pressure that would allow the DNAPL to drain and water to enter the hydrophobic sand. The thickness or depth of the trapped NAPL layer is similar in lenses I and II if you consider the bottom of that layer as the lowermost hydrophobic-hydrophilic soil interface. 
It is also clear from the NAPL distribution in Figure 36 that the DNAPL drained from the lowermost hydrophobic-hydrophilic soil interfaces in both lenses I and II. These were the locations of the plastic dividers during sand placement. It also appears that the DNAPL invaded more pores in the hydrophilic porous media as it migrated from lens I to lens II during the surfactant flush, which is consistent with a decrease in interfacial tension allowing the NAPL to enter more pores and reducing the impact of soil heterogeneity. An estimate of the DNAPL distribution after the surfactant flush based on the areas outlined in the image on the right in Figure 36 indicates that $10 \mathrm{~mL}$ remained in the spill zone and approximately $10 \mathrm{~mL}$ and $25 \mathrm{~mL}$ remained in the saturated portions of lenses I and II, respectively. The remaining $30 \mathrm{~mL}$ should be held as residual in the hydrophilic sand (light blue areas) and drained portions of the hydrophobic lenses (medium blues areas) and a portion removed from the box. If the residual NAPL saturation is assumed to be $20 \%$, approximately $23 \mathrm{~mL}$ of NAPL is held in these zones and $7 \mathrm{~mL}$ was removed via the surfactant flush. No pure phase liquid was removed from the box or found in the well at either edge of the box. Effluent concentrations were not monitored; however, if you assume that the 7 pore volumes of effluent recovered during the surfactant flush were saturated with the DNAPL compounds $(1428 \mathrm{mg} / \mathrm{L}$ based on the composition of the DNAPL), this would amount to approximately $9 \mathrm{~mL}$ of DNAPL. Therefore, one can conceptually arrive at a reasonable mass balance. 


\section{DISCUSSION}

The flow patterns seen in these experiments were similar to those found in other work (Oostrom et al. 1999a; Conrad et al. 2002; O'Carroll et al. 2004; Zheng et al. 2016). When the DNAPL was injected into the saturated hydrophilic material, there was pooling at the interface between the sand and water. The height of the DNAPL pool increased until the entry pressure was achieved, at which point the DNAPL began its downward migration. The exact pattern of downward migration was slightly different in each trial. This is a common effect in subsurface flow experiments as it is very difficult to exactly replicate the packing of the porous medium and NAPLs will flow through preferential flow paths (Conrad et al. 2002). In addition, the type of porous media in the box did affect both the migration and remediation of the DNAPL in the box.

As expected the spill in the hydrophilic only material resulted in the DNAPL migrating downward with very little lateral spread until it came into contact with the fine grained material at the bottom of the box (Taylor et al. 2001, Zheng et al. 2015, Zheng et al. 2016). As the DNAPL pooled at the bottom of the box it began to spread laterally across the bottom of the box. As a result of the lateral spread the DNAPL was able to enter the manifolds at the sides of the box and this resulted in the recovery of pure phase DNAPL during the waterflood. The pressure transducer data support the migration pattern, with the bulk of the DNAPL traveling through the centre of the box. This meant that the majority of transducers never came into contact with the DNAPL and the transducers at the bottom of the box were placed too high to record any of the pooling that occurred as the DNAPL came into contact with the fine grained layer. Although the spills in this work were conducted under static conditions, work by Zheng et al. (2015) 
found that in homogeneous media the lateral and vertical migration of a DNAPL spill was significantly altered by increasing flow velocity.

The pattern of migration in the hydrophilic media with fine grained lenses was similar to that seen in other works (Taylor et al. 2001, Conrad et al. 2002, Suchomel et al. 2007, Zheng et al. 2016). When in contact with the fine grained lenses, the DNAPL pooled at the point of contact and then began to spread laterally across the lens until it reached the edge of the lens where it continued its downward migration. As in the experiments with no lenses some pure phase DNAPL was collected during the waterflood. As with the homogeneous medium the pressure transducer data supported the photos indicating that the DNAPL did not enter the fine grained material in any of the trials. Other work has shown that if the pools of DNAPL which form on the fine grained material reach the entry pressure there is the possibility that the DNAPL could enter the fine grained lens (Kueper et al. 1989).

The experiments with the hydrophobic lenses were the only ones where the DNAPL did not reach the bottom of the box in all the trials. Similar to the results previously seen (O'Carroll et al. 2004), the hydrophobic lenses draw in the mobile phase of the DNAPL, and can actually retard (lens I) or even completely halt (lens II), the downward migration of the DNAPL. In addition, the DNAPL that is drawn into the lenses is removed from the mobile phase, which can reduce the depth of the DNAPL penetration in the subsurface and the area of contamination. As in the previous experiments, the pressure transducer data supported the photographs taken over the course of the experiments. The capillary pressure increased very quickly as the DNAPL was drawn into the hydrophobic lenses. 
For all experiments when a waterflood was conducted, the changes to the DNAPL saturations and distributions were limited. This result was expected as previous experiments and extensive field data have shown that waterfloods alone are not very successful in remediating sites contaminated with DNAPL and are particularly ineffective in the presence of hydrophobic soils (Anderson 1987, Dwarakanath et al. 2002). This was seen in these experiments as the only pure phase DNAPL was recovered at the start of the waterflood and only in the experiments with no hydrophobic media. It was likely the majority of the DNAPL in these experiments was removed as a result of becoming trapped in the side manifolds.

In contrast to the waterflood results, the surfactant flush resulted in significant redistribution of the DNAPL within the box in all experiments. Surfactant flushes have been used with success in DNAPL removal under a variety of conditions. Taylor et al. (2001) used a 4\% Tween 80 solution to remediate batch, column and two-dimension heterogeneous boxes contaminated with PCE. They achieved recoveries of 70-80.5\% under different flow scenarios and any remaining PCE was perched on lenses of lower permeability material. In contrast, Conrad et al. (2002) found that a 5\% Tween 80 solution and a $1.6 \%$ Aerosol MA, $4 \%$ propanol and $0.3 \% \mathrm{NaCl}$ solution did remove a large percentage of the DNAPL perched on media with lower permeability. As the surfactant solution drained the pools of DNAPL, the TCE followed the previous migration pattern of the original spill. However, during the Aerosol MA flush, there was unwanted migration of the TCE into the aquitard at the base of the box. This unwanted migration was seen in these experiments, as the saturation of DNAPL between lenses increased and some of the DNAPL reached the bottommost lens that had remained 
uncontaminated during the previous two phases of the experiment. In fact, in a review of surfactant enhanced remediation conducted by Mulligan et al. (2001), it was determined that although the addition of surfactants can accelerate remediation, some of the unintended consequences of the remediation is the migration of the DNAPL into previously uncontaminated areas and uneven treatment in the contaminated area.

The use of pressure transducers to monitor NAPL spills in laboratory experiments is not common. This is likely due to the difficulty in both the setup and the analysis of the data. Using pressure transducers to understand the pressures that govern the distribution and migration of NAPLs in a NAPL-wet medium is unique. The pressure data confirmed that the DNAPL will be drawn into the NAPL-wet lenses and result in negative capillary pressures as defined in Bradford and Leij (1995) and O'Carroll et al. (2005). The pressure data also indicated that NAPL may become trapped within NAPL-wet lenses and that for the NAPL to drain from a NAPL-saturated lens, not only must it overcome the NAPL-entry pressure of the soil below but the water pressure must exceed the water entry pressure of the NAPL-wet soil in order for the NAPL to drain.

Experimental data is important when selecting the optimal remediation techniques and can depend on a number of factors. In a review of the use of soil flushing as a remediation technique, Atteia et al. (2013) found that there is no one technique for every situation and that a good knowledge of the subsurface environment and pollutant are necessary to ensure a successful remediation. The results presented herein have implications for remediation in the field and help explain why removal levels in the field are less than predicted. In addition to the impacts of heterogeneity in terms of permeability and porosity, heterogeneity in wettability will impact the NAPL distribution 
and removal levels via surfactant flushing. The pressure transducer data within the hydrophobic lens for the simulated spill indicate that NAPL will be drawn into these lenses and become effectively trapped within the lenses preventing further downward migration. While surfactants may decrease the interfacial tension of the water-NAPL fluid pair reducing the capillary forces and potentially allowing the NAPL to drain from these lenses, not all the NAPL will drain and a NAPL saturated layer will remain within the lens or above the hydrophilic-hydrophobic interface. The thickness of that layer will reflect the reduced NAPL entry pressure of the hydrophilic soil and the reduced water entry pressure of the hydrophobic soil.

\section{CONCLUSIONS}

\subsection{Conclusions}

A series of DNAPL spills, both TCE and a field sample, were conducted in a twodimensional sand box, filled with a variety of porous media, including hydrophilic porous media, hydrophilic porous media with fine grained hydrophilic lenses and hydrophilic porous medium with hydrophobic lenses. The spills were monitored by photography and pressure transducers. The migration patterns and remediation of both the field DNAPL and TCE were similar. This was not unexpected as the chemical analysis of the field sample suggested that it was unused product and had properties similar to pure TCE. Regardless of the porous media after the entry pressure was achieved, the DNAPL traveled downwards until it came into contact with either fine grained media or a hydrophobic lens. The DNAPL pooled above the fine grained lens and the DNAPL was 
drawn into the hydrophobic lens. The pressure transducer data was used to corroborate the photographic data. This information was most useful in the experiments with the hydrophobic lenses for which the pressure transducer data confirmed that the capillary pressures became negative within a hydrophobic lens and appeared to follow a capillary pressure-saturation relationship which mirrored that of the hydrophilic sand, only negative. The pressure transducer data also indicated that NAPL may become trapped in a hydrophobic lens even though the NAPL pressure may exceed the NAPL entry pressure of the underlying hydrophilic soil. This may be due to the fact that the NAPL could not drain from the hydrophobic soil unless the water pressure exceeded the water entry pressure of the hydrophobic soil. The thickness of an entrapped NAPL zone within a hydrophobic lens above a hydrophilic soil appears to be governed by the NAPL entry pressure of the underlying hydrophilic soil and water entry pressure of the hydrophobic soil which allows the NAPL to drain. A waterflood had little impact on the distribution of the NAPL within the soil. A surfactant flush was able to solubilise and re-mobilize the NAPL in all the experiments. This resulted in the removal of some of the pooled DNAPL in the hydrophilic media and the hydrophilic media with fine grained lenses. For the hydrophobic soil, the reduction in the interfacial tension of the water-DNAPL fluid pair reduced the NAPL entry pressure of the hydrophilic soil and the water entry pressure of the hydrophobic lens. An entrapped DNAPL layer remained in the lower portion of the hydrophobic lens above the underlying hydrophilic soil; however, the surfactant, and the corresponding reduction in interfacial tension, was able to reduce the thickness of the entrapped DNAPL layer. It is thought that by reducing the interfacial tension between the NAPL and water to 0 it would be possible to obtain complete 
drainage of hydrophobic soils. This will have implications for remediation in the field in that the added heterogeneity due to wettability variations may lower the removal efficiency of NAPLs for the aforementioned reasons.

\subsection{Recommendations}

This work has shown that pressure transducers can be used to collect important data on the behaviour of DNAPLs in the subsurface. However, based on the results of these experiments some suggestions can be made:

- Laboratory grade DNAPLs can serve as a substitute for field DNAPLs under certain conditions, particularly when the field DNAPL has properties including the density, viscosity and interfacial tension which are similar to the laboratory DNAPL to be used as a substitute.

- Although the pressure transducers did provide important data they were most useful during the experiments with the hydrophobic lenses. This is due to the fact that in the majority of the experiments the DNAPL never came in contact with the transducers. By decreasing the space between transducers and increasing the number of overall transducers there is a greater chance of contact between a transducer and the DNAPL. In addition, the placement of the pressure transducer can be better designed, perhaps after a few trial runs. To improve the probability of obtaining useful DNAPL pressure readings the water transducers could be replaced by DNAPL transducers as the pressure within the water transducers remained relatively constant at the elevation pressure throughout the experiments. 
In the future I would suggest that all the transducers record DNAPL pressures and to obtain the capillary pressure the height of the transducer is assumed to be the water pressure.

- Decreasing the interfacial tension between the water and DNAPL to zero to confirm that this will result in the complete drainage of the hydrophobic lenses.

- The porous media used in these experiments were all very uniform which results in relative flat capillary pressure-saturation curve. With a flat curve small change to capillary pressure will result in large changes to the saturation of the porous medium. The errors associated with these experiments include the pressure transducers themselves and errors during calibration. Due to the relatively flat nature of the curves associated with the media used in these experiments even these small errors can have a large impact on the calculated level of saturation at any given location within the box. More accurate calibration procedures and transducers could help reduce these effects.

- Research (Seyedabbasi et al. 2012 and Wilking et al. 2013) has shown that although the majority of DNAPL can be removed from the subsurface fine grained material can act as a contaminant source for a number of years. In this work no DNAPL entered the fine grained lenses, so future work in which the pressure could be monitored within contaminated fine grained lenses would likely produce beneficial results.

- No in depth image analysis was performed for these experiments as the images were not of sufficient quality. This is a common drawback of light reflection methods, as reviewed by Oostrom et al. (2007). In these experiments the lighting 
saturation across the front of the box was hard to maintain and these minute shifts in lighting resulted in change to the colour saturation levels and made distinguishing minute changes in saturation difficult. A more accurate image acquisition system, such as gamma or X-ray methods, may provide improved precision in determining the movement of the DNAPL within the sand box. 


\section{REFERENCES}

Abdallah, W., Buckley, J.S., Carnegie, A., Edwards, J., Herold, B., Fordham, E., Graue, A., Habashy, T., Seleznev, N., Signer, C., Hussain, H., Montaron, B., and Ziauddin, M. 2007. Fundamentals of wettability. Oilfield Review; 19(2): 44-61.

Abriola, L.M. 1989. Modelling multiphase migration of organic chemicals in groundwater systems - A review and assessment. Environmental Health Prespectives; 88: $117-143$.

Abriola, L.M., Rathfelder, K., Maiza, M., and Yadav, S. 1992. VALOR code version 1.0: A PC code for simulating immiscible contaminant transport in subsurface systems. EPRI TR-101018, Project 2879-08.

Ahmed, M.E., and van Geel, P.J. 2009. Potential concerns related to using octadecyltrichlorosilane (OTS) in rendering soils and porous ceramics hydrophobic. Journal of Contaminant Hydrology; 110: 22-33.

Al-Raoush, R.I. 2009. Impact of wettability on pore-scale characteristics of residual nonaqueous phase liquids. Environmental Science \& Technology; 43(13): 4796-4801.

Anckner, W.H. Jr., and Powers, S.E. 1996. Relating the wettability of contaminated sands to NAPL composition. Non-Aqueous Phase Liquids (NAPLs) in Subsurface Environment: Assessment and Remediation, ASCE Specialty Conference Proceedings, 502-512.

Anderson, W.G. 1987a. Wettability literature survey - Part 4: Effects of wettability on capillary pressure. Journal of Petroleum Technology; 1283-1300.

Anderson, W.G. 1987b. Wettability literature survey - Part 6: The effects of wettability on waterflooding. Journal of Petroleum Technology; 1605-1620.

Atteia, O., Estrada, E.D.C., Bertin, H. 2013. Soil flushing: a review of the origin of efficiency variability. Reviews in Environmental Science and Bio/Technology; 12:379389.

Bedient, P.B., Rifai, H.S., and Newell, C.J. 1997. Ground water contamination Transport and remediation. Prentice Hall PTR, Upper Saddle River, NJ.

Bradford, S.A., Abriola, L.M., and Rathfelder, K.M. 1998. Flow and entrapment of dense nonaqueous phase liquids in physically and chemically heterogeneous aquifer formations. Advances in Water Resources; 22(2): 117-132.

Bradford, S.A. and Leij, F.J. 1995. Fractional wettability effects on two- and three-fluid capillary pressure-saturation relations. Journal of Contaminant Hydrology; 20: 89-109. 
Brooks, R.H., and Corey, A.T. 1964. Hydraulic properties of porous media. Hydrology Papers No 3, Civil Engineering Department, Colorado State University, Fort Collins.

Burdine, N.T. 1953. Relative permeability calculations from pore size distribution data. Transactions of the American Institute of Mining and Metallurgical Engineering; 198: 71-78.

Canadian Council of Ministers of the Environment (CCME). 2008. National Classification System for Contaminated Sites - Guidance Document. PN 1403; ISBN 978-1-896997-80-3.

Childs, J., Acosta, E., Annable, M.D., Brooks, M.C., Enfield, C.G., Harwell, J.H., Hasegawa, M., Knox, R.C., Rao, P.S.C., Sabatini, D.A., Shiau, B., Szekeres, E., and Wood, A.L. 2006. Field demonstration of surfactant-enhanced solubilisation of DNAPL at Dover Air Force Base, Deleware. Journal of Contaminant Hydrology; 82: 1-22.

COMSOL. 2008. COMSOL Multiphysics modeling guide; version November 2008, COMSOL 3.5a.

Conrad, S.H., Glass, R.J., and Peplinski, W.J. 2002. Bench-scale visualization of DNAPL remediation processes in analog heterogeneous aquifers: surfactant floods and in situ oxidation using permanganate. Journal of Contaminant Hydrology; 58: 13-49.

Craig, F.F. Jr. 1971. The reservoir engineering aspects of waterflooding. Society of Petroleum Monograph Series, 3.

Darnault, C.J.G., Throop, J.A., DiCarlo, D.A., Rimmer, A., Steenhuis, T.S., and Parlange, J.Y. 1998. Visualization by light transmission of oil and water contents in transient twophase flow fields. Journal of Contaminant Transport; 31: 337-348.

Dekker, T.J., and Abriola, L.M. 2000. The influence of field-scale heterogeneity on the infiltration and entrapment of dense nonaqueous phase liquids in saturated formations. Journal of Contaminant Hydrology; 42:187-218.

Denekas, M.O., Mattax, C.C., and Davis, G.T. 1959. Effects of crude oil components on rock wettability. Journal of Petroleum Technology; 11(11): 330-333.

Dou, W., Omran, K., Grimberg, S.J., Denham, M., and Powers, S.E. 2008. Characterization of DNAPL from the U.S. DOE Savannah River Site. Journal of Contaminant Hydrology; 97:75-86.

Dwarakanath, V., Jackson, R.E., and Pope, G.A. 2002. Influence of wettability on the recovery of NAPLs from alluvium. Environmental Science \& Technology; 36:227-231.

Dwarankanth, V., Kostarelos, K., Pope, G.A., Shotts, D., and Wade, W.H. 1999. Anioinc surfactant remediation of soil columns contaminated by nonaqueous phase liquids. Journal of Contaminant Hydrology; 38: 465-488. 
Dwarakanath, V., and Pope, G.A. 2000. Surfactant phase behavior with field degreasing sovent. Environmental Science \& Technology; 34:4842-4848.

Federal Contaminated Sites Action Plan (FCSAAP). 2008. Federal contaminated sites accelerated action plan: Annual report 2003-2004. ISBN Enl-43/2004E-PDF.

Fountain, J.C., Klimek, A., Beikirch, M.G., and Middleton, T.M. 1991. The use of surfactants for in situ extraction of organic pollutants from a contaminated aquifer. Journal of Hazardous Materials; 28: 295-311.

Franzetti, A., Di Gennaro, P., Bevilacqua, A., Papacchini, M., and Bestetti, G. 2006. Environmental features of two commercial surfactants widely used in soil remediation. Chemosphere; 62: 1474-1480.

Goswami, R.R., Ambale, B., and Clement, T.P. 2009. Estimating errors in concentration measurements obtained from image analysis. Vadose Zone Journal; 8(1): 108-118.

Harrold, G., Goody, D.C., Lerner, D.N., and Leharne, S.A. 2001. Wettability changes in trichloroethylene-contaminated sandstone. Environmental Science and Technology; 35: 1504-1510.

Harrold, G., Goody, D.C., Reid, S., Lerner, D.N., and Leharne, S.A. 2003. Changes in interfacial tension of chlorinated solvents following flow through U.K. soils and shallow aquifer material. Environmental Science \& Technology; 37:1919-1925.

Harrold, G., Lerner, D.N., and Leharne, S.A. 2005. The impact of additives found in industrial formulations of TCE on the wettability of sandstone. Journal of Contaminant Hydrology; 80: 1-17.

Health Canada. 2008. Guidelines for Canadian drinking water quality summary table. Prepared by the Federal-Provincial-Territorial Committee on Drinking Water.

Hoa, N.T. 1981. A new method allowing the measurement of rapid variations of the water content in sandy porous media. Water Resources Research; 17(1): 41-48.

Jafvert, C.T. 1996. Report: Surfactants/Cosolvents. Ground-Water Remediation Technologies Analysis Center, Document TE-96-02.

Jeong, S.-W., Wood, A.L., and Lee, T.R. 2002. Effects of pure and dyed PCE on physical and interfacial properties of remedial solutions. Journal of Hazardous Material; B95:125-135.

Jin, M., Hirasaki, G.J., Jackson, R.E., Kostarelos, K., and Pope, G.A. 2007. Control of downward migration of dense nonaqueous phase liquids during surfactant flooding by design simulations. Water Resources Research; 43, W01412.

King, J.A. 2005. Digital photography for dummies, $5^{\text {th }}$ edition. Wiley Publishing, Inc. Indianapolis, Indiana. 
Kim, I.S., Park, J.-S., and Kim, K.-W. 2001. Enhanced biodegradation of polycyclic aromatic hydrocarbons using non-ionic surfactants in soil slurry. Applied Geochemistry; 16: $1419-1428$.

Kueper, B.H., Abbott, W., and Farquhar, G. 1989. Experimental observations of multiphase flow in heterogeneous porous media. Journal of Contaminant Hydrology; 5:83-95.

Kueper, B.H., Wealthall, G.P, Smith, J.W.N., Leharne, S.A., and Lerner, D.N. 2003. An illustrated handbook of DNAPL transport and fate in the subsurface. Environment Agency, R\&D Publication 133.

Lemke, L.D., and Abriola, L.M. 2006. Modelling dense nonaqueous phase liquid mass removal in nonuniform formations: Linking source-zone architecture and system response. Geosphere; 2(2): 74-82.

Lowe, D.F., Oubre, C.L., and Ward, C.H. 1998. Surfactants and cosolvents for NAPL remediation: A technology practices manual. Lewis Publishers; Boca Raton, FL.

Mayer, A., and Hassanizadeh, S.M. 2005. Soil and groundwater contamination: nonaqueous phase liquids - principles and observations. Water Resources Monograph 17. American Geophysical Union, Washington, DC.

Mercer, J.W., and Cohen, R.M. 1990. A review of immiscible fluids in the subsurface: properties, models, characterization and remediation. Journal of Contaminant Hydrology; 6: 107-163.

Miller, C.T., Christakos, G., Imhoff, P.T., McBride, J.F., Pedit, J.A., and Trangenstein, J.A. 1998. Multiphase flow and transport modelling in heterogeneous porous media: challenges and approaches. Advances in Water Resources; 21(2): 77-120.

Mualem, Y. 1976. A new model for predicting the hydraulic conductivity of unsaturated porous media. Water Resources Research; 12: 513-522.

National Round Table on the Environment and the Economy (NRTEE). 2003. Cleaning up the past, building the future - A national brownfield redevelopment strategy for Canada. Renouf Publishing Co. Ltd. Ottawa, Canada.

Niemet, M.R., and Selker, J.S. 2001. A new method for quantification of liquid saturation in 2D translucent porous media systems using light transmission. Advances in Water Resources; 24: 651-666.

O'Carroll, D.M, Abriola, L.M., Polityda, C.A., Bradford, S.A., and Demond, A.H. 2005. Prediction or two-phase capillary pressure-saturation relationships in fractional wettability systems. Journal of Contaminant Hydrology; 77: 247-270. 
O'Carroll, D.M., Bradford, S.A., and Abriola, L.M. 2004. Infiltration of PCE in a system containing spatial wettability variations. Journal of Contaminant Hydrology; 73:39-63.

Oolman, T., Godard, S.T., Pope, G.A., Jin, M., and Kirchner, K. 1995. DNAPL flow behavior in a contaminated aquifer: Evaluation of field data. Ground Water Monitoring \& Remediation; 15(4):125-137.

Oostrom, M., Dane, J.H., and Wietsma, T.W. 2007. A review of multidimensional, multifluid, intermediate-scale experiments: flow behaviour, saturation imaging, and tracer detection and quantification. Vadose Zone Journal; 6(3): 610-637.

Oostrom, M., Hofstee, C.,Walker, R.C., and Dane, J.H. 1999a. Movement and remediation of trichloroethylene in a saturated heterogeneous porous medium 1. Spill behavior and initial dissolution. Journal of Contaminant Hydrology; 37:159-178.

Oostrom, M., Hofstee, C.,Walker, R.C., and Dane, J.H. 1999b. Movement and remediation of trichloroethylene in a saturated heterogeneous porous medium 2. Pumpand-treat and surfactant flushing. Journal of Contaminant Hydrology; 37:179-197.

Pankow, J.F., and Cherry, J.A. 1996. Dense chlorinated solvents and other DNAPLs in groundwater: History, Behavior, and Remediation. Waterloo Press, Portland, Oregon.

Pennell, K.D., Pope, G.A., and Abriola, L.M. 1996. Influences of viscous and buoyancy forces on the mobilization of residual tetrachloroethylene during surfactant flushing. Environmental Science \& Technology; 30: 1328-1335.

Powers, S.E., Anckner, W.H., and Seacord, T.F. 1996. Wettability of NAPLcontaminated sands. Journal of Environmental Engineering; 122(10): 889-896.

Powers, S.E., and Tamblin, M.E. 1995. Wettability of porous media after exposure to synthetic gasolines. Journal of Contaminant Hydrology; 19: 105-125.

Rathfelder, K.M, and Abriola, L.M. 1998. The influence of capillarity in numerical modeling of organic liquid redistribution in two-phase systems. Advances in Water Resources; 21(2): 159-170.

Rathfelder, K.M, Abriola, L.M., Singletary, M.A., and Pennell, K.D. 2003. Influence of surfactant-facilitated interfacial tension reduction on chlorinated solvent migration in porous media: observations and numerical simulation. Journal of Contaminant Hydrology; 64: 227-252.

Schincariol, R.A., Herderick, E.E., and Schwartz, F.W. 1993. On the application of image analysis to determine concentration distributions in laboratory experiments. Journal of Contaminant Hydrology; 12: 197-215. 
Schincariol, R.A., and Schwartz, F.W. 1990. An experimental investigation of variable density flow and mixing in homogeneous and heterogeneous media. Water Resource Research; 26(10): 2317-2329.

Schwille, F. 1988. Dense chlorinated solvents in porous fractured media. Lewis Publishers, Inc. Chelsea, Michigan.

Seo, H.S., and McCray, J.E. 2002. Interfacial tension of chlorinated aliphatic DNAPL mixtures as a function of organic phase composition. Environmental Science \& Technology; 36:1292-1298.

Seyedabbasi, M.A., Newell, C.J., Adamson, D.T. and Sale, T.C. 2012. Relative contribution of DNAPL dissolution and matrix diffusion to the long-term persistence of chlorinated solvent source zones. Journal of Contaminant Hydrology; 134-135:69-81.

Singha, K., and Loheide, S.P. II. 2011. Linking physical and numerical modeling in hydrogeology using sand tank experiments and COMSOL Multiphysics. International Journal of Science Education; 34(4): 547-571.

Suchomel, E.J., Ramsburg, A., Pennell, K.D. 2007. Evaluation of trichloroethene recovery processes in heterogeneous aquifer cells flushed with biodegradable surfactants. Journal of Contaminant Hydrology; 94: 195-214.

Taylor, T.P., Pennell, K.D., Abriola, L.M., and Dane, J.H. 2001. Surfactant enhanced recovery of tetrachloroethylene from a porous medium containing low permeability lenses. 1. Experimental studies. Journal of Contaminant Hydrology; 48: 325-350.

Thakur, S., Schenewerk, P., Wolcott, J., Groves, F.Jr. 1995. Flow characteristics of DNAPLs in sand packs: Water-influx displacement. Journal of Environmental Science and Health; A30(5): 1105-1118.

Tuck, D.M., Iversen, G.M., and Pirkle, W.A. 2003. Organic dye effects on dense nonaqueous phase liquids (DNAPL) entry pressure in water saturated porous media. Water Resources Research; 39(8), 1207.

Van Geel, P.J., and Sykes, J.F. 1994. Laboratory and model simulations of a LNAPL spill in a variably-saturated sand: 1. Laboratory experiment and image analysis techniques. Journal of Contaminant Hydrology; 25: 249-270.

Van Genuchten, M.T. 1980. A closed-form equation for predicting the hydraulic conductivity of unsaturated soils. Soil Science Society of America Journal; 44:892-898.

White, R. 2006. How digital photography works. Que Publishing, Indianapolis, Indiana.

Wilking, B.T., Rodriguez, D.R. and Illangasekare, T.H. 2013. Experimental study of the effects of DNAPL distribution on mass rebound. Groundwater; 51(2):229-236. 
Zheng, F., Gao, Y., Sun, Y., Shi, X., Xu, H., Wu, J. 2015. Influence of flow velocity and spatial heterogeneity on DNAPL migration in porous media: insights from laboratory experiments and numerical modelling. Hydrogeology Journal; 231:1703-1718.

Zheng, F., Gao, B., Sun, Y., Shi, X., Xu, H., Wu, J. and Gao, Y. 2016. Removal of tetrachloroethylene from homogeneous and heterogeneous porous media: Combined effects of surfactant solubilisation and oxidant degradation. Chemical Engineering Journal; 283:595-603. 